\title{
REPRODUCING KERNEL HILBERT SPACES
}

\author{
A THESIS \\ SUBMITTED TO THE DEPARTMENT OF MATHEMATICS \\ AND THE INSTITUTE OF ENGINEERING AND SCIENCE \\ OF BILKENT UNIVERSITY \\ IN PARTIAL FULFILLMENT OF THE REQUIREMENTS \\ FOR THE DEGREE OF \\ MASTER OF SCIENCE
}

By

Baver Okutmuştur

August, 2005 
I certify that I have read this thesis and that in my opinion it is fully adequate, in scope and in quality, as a thesis for the degree of Master of Science.

\section{Assist. Prof. Dr. Aurelian Gheondea (Supervisor)}

I certify that I have read this thesis and that in my opinion it is fully adequate, in scope and in quality, as a thesis for the degree of Master of Science.

Prof. Dr. Mefharet Kocatepe

I certify that I have read this thesis and that in my opinion it is fully adequate, in scope and in quality, as a thesis for the degree of Master of Science.

Assoc. Prof. Dr. H. Turgay Kaptanoğlu

Approved for the Institute of Engineering and Science:

Prof. Dr. Mehmet B. Baray

Director of the Institute Engineering and Science 


\title{
ABSTRACT REPRODUCING KERNEL HILBERT SPACES
}

\author{
Baver Okutmuştur \\ M.S. in Mathematics \\ Supervisor: Assist. Prof. Dr. Aurelian Gheondea
}

August, 2005

\begin{abstract}
In this thesis we make a survey of the theory of reproducing kernel Hilbert spaces associated with positive definite kernels and we illustrate their applications for interpolation problems of Nevanlinna-Pick type. Firstly we focus on the properties of reproducing kernel Hilbert spaces, generation of new spaces and relationships between their kernels and some theorems on extensions of functions and kernels. One of the most useful reproducing kernel Hilbert spaces, the Bergman space, is studied in details in chapter 3. After giving a brief definition of Hardy spaces, we dedicate the last part for applications of interpolation problems of NevanlinnaPick type with three main theorems: interpolation with a finite number of points, interpolation with an infinite number of points and interpolation with points on the boundary. Finally we include an Appendix that contains a brief recall of the main results from functional analysis and operator theory.
\end{abstract}

Keywords: Reproducing kernel, Reproducing kernel Hilbert spaces, Bergman spaces, Hardy spaces, Interpolation, Riesz theorem. 


\title{
ÖZET \\ DOĞURAN ÇEKIRDEKLI HILBERT UZAYLARI
}

\author{
Baver Okutmuştur \\ Matematik, Yüksek Lisans \\ Tez Yöneticisi: Yrd. Doç. Dr. Aurelian Gheondea
}

Ağustos, 2005

$\mathrm{Bu}$ tezde, doğuran çekirdekli Hilbert uzayları teorisini pozitif tanımlı çekirdekler ile beraber inceledik ve bunun uygulamalarını Nevallina-Pick interpolasyon problemleri üzerinde örnekledik. Öncelikle, doğuran çekirdekli Hilbert uzaylarının özelliklerini, üretilen yeni uzaylar ve onların çekirdekleri arasındaki ilişkileri ve genişletilen çeşitli fonksiyon ve çekirdeklerle ilgili bazı teoremleri inceledik. Sıkça kullanılan doğuran çekirdekli Hilbert uzaylarından biri olan Bergman uzayı 3. kısımda detaylarıyla işlendi. Hardy uzayının kısa bir tanımıyla başladığımız son kısım, Nevallina-Pick interpolasyon problemlerinin uygulamalarını içeren üç ana teorem ile son buldu. Bunlar: sınırlı sayıda nokta ile interpolasyon, sinırsız sayıda nokta ile interpolasyon ve sinır noktalarında interpolasyon. Son olarak Appendix kısmı bu tezde sıkça kullandıg̃ımız fonksiyonel analiz ve operator teori ile ilgili temel esasların kısa bir özetine ayrıldı.

Anahtar sözcükler: Doğuran çekirdekler, Doğuran çekirdekli Hilbert uzayları, Bergman uzayları, Hardy uzayları, Interpolasyon, Riesz teoremi. 


\section{Acknowledgement}

I would like to express my sincere gratitude to my supervisor Assoc. Prof. Aurelian Gheondea, firstly for introducing me to this area of analysis, for his excellent guidance, valuable suggestions, encouragement and infinite patience. I am glad to have a chance to study with this great person who is a role model as a supervisor and a mathematician.

My sincere thanks to Prof. Dr. Mefharet Kocatepe for her interest and useful comments.

I also need to express my gratitude to Assoc. Prof. H. Turgay Kaptanog̃lu for providing me necessary documents for this study.

I am grateful to Aslı Pekcan and Murat Altunbulak who helped me about Latex. Special thanks to Aslı for reading my thesis, for her advices, corrections and helps.

Beside, I would like to thank Serdar, Hikmet, Rohat, Ergün, İnan, Fatma, Burcu, Sultan and all my friends who always cared about my work and increased my motivation, which I have strongly needed.

I would like to thank Eliya Büyükkaya who has always been with me with her endless love, support and understanding.

Finally, I would like to express my deepest gratitude for the constant support, encouragement, trust and love that I received from my family. 


\section{Contents}

1 Introduction $\quad 1$

2 Reproducing Kernel Hilbert Spaces $\quad 4$

2.1 Definition, Uniqueness and Existence . . . . . . . . . . . 4

2.2 Operations with Reproducing Kernel Hilbert Spaces . . . . . . . . 15

2.3 Extension of Functions and Kernels . . . . . . . . . . . . . . . 25

3 Spaces of Analytic Functions 33

3.1 Sesqui-analytic kernels . . . . . . . . . . . . . . . . . . . 33

3.2 Bergman Spaces . . . . . . . . . . . . . . . . . . 42

3.3 Szegö Kernel . . . . . . . . . . . . . . . . . . . . . . . . . . . . . 48

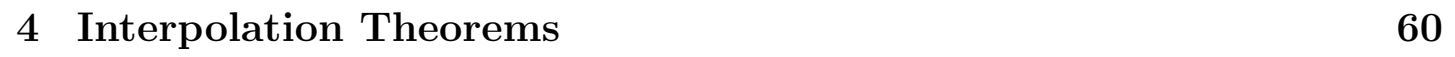

4.1 General definition of Hardy spaces . . . . . . . . . . . . . . . . . . 60

4.2 Interpolation Inside Unit Disc . . . . . . . . . . . . . . . . . . 66

4.3 Interpolation on the Boundary . . . . . . . . . . . . . . . 73 
\begin{tabular}{ll}
\hline A Hilbert Spaces & 76
\end{tabular}

A.1 Definitions . . . . . . . . . . . . . . 76

A.2 Projection ......................... 83

A.3 Weak Topology . . . . . . . . . . . . . . . . . . . . . 88

A.4 Self-adjoint Operators . . . . . . . . . . . . . . . . . 91 


\section{Chapter 1}

\section{Introduction}

The reproducing kernel was used for the first time at the beginning of the 20th century by S. Zaremba in his work on boundary value problems for harmonic and biharmonic functions. In 1907, he was the first who introduced, in a particular case, the kernel corresponding to a class of functions, and stated its reproducing property. But he did not develop any theory and did not give any particular name to the kernels he introduced.

In 1909, J. Mercer examined the functions which satisfy reproducing property in the theory of integral equations developed by Hilbert and he called this functions as 'positive definite kernels'. He showed that this positive definite kernels have nice properties among all continuous kernels of integral equations.

However, for a long time these results were not investigated. Then the idea of reproducing kernels appeared in the dissertations of three Berlin mathematicians G. Szegö (1921), S. Bergman (1922) and S. Bochner (1922). In particular, S. Bergman introduced reproducing kernels in one and several variables for the class of harmonic and analytic functions and he called them 'kernel functions'.

In 1935, E.H. Moore examined the positive definite kernels in his general analysis under the name of positive Hermitian matrix.

Later, the theory of reproducing kernels was systematized by N.Aronszajn 
around 1948.

The original idea of Zaremba to apply the kernels to the solution of boundary value problems was developed by S. Bergman and M. Schiffer. In these investigations, the kernels were proved to be powerful tool for solving boundary value problems of partial differential equations of elliptic type. Moreover, by application of kernels to conformal mapping of multiply-connected domains, very beautiful results were obtained by S. Bergman and M. Schiffer.

Several important results were achieved by the use of these kernels in the theory of one and several complex variables, in conformal mapping of simplyand multiply-connected domains, in pseudo-conformal mappings, in the study of invariant Riemannian metrics and in other subjects.

Meanwhile, in probability theory, the theory of positive definite kernels was used by A.N. Kolmogorov, E. Parzen and others.

There are also several papers and lecture notes on this subject; B. Burbea (1987), E. Hille (1972), S. Saitoh (1988), H. Dym (1989) and T. Ando (1987). Most part of this thesis owes to T. Ando's lecture notes [1] in its diversity of tools and results. We also used H. Dym, S. Saitoh and N. Aronszajn's works especially for the second chapter. Moreover, we used partially the books of P.L. Duren [4], P. Koosis [7], P.L. Duren and A. Schuster's [5] for complementing with result on Bergman and Hardy spaces.

The thesis is organized as follows:

In Chapter 2, after giving definitions and properties of reproducing kernel Hilbert spaces with some theorems, we focus on generation of new spaces and relationship between their kernels. Also, some extension theorems of functions and kernels are proven.

In Chapter 3, we present some of the most useful reproducing kernel Hilbert spaces consisting of analytic functions. A special role is played by the Bergman spaces and Bergman kernels that we present in detail. 
Chapter 4 is dedicated to applications to interpolation problems of Nevanlinna-Pick type. We start with a brief definition of Hardy spaces. Then we prove three main theorems: interpolation with a finite number of points, interpolation with an infinite number of points, and interpolation with points on the boundary.

The Appendix part contains some elementary facts from functional analysis and operator theory in Hilbert spaces which can be found in textbooks, e.g. in J. Conway [3] and J. Weidman [9]. 


\section{Chapter 2}

\section{Reproducing Kernel Hilbert Spaces}

\subsection{Definition, Uniqueness and Existence}

Definition 2.1.1. Let $\mathcal{H}$ be a Hilbert space of functions on a set $X$. Denote by $\langle f, g\rangle$ the inner product and let $\|f\|=\langle f, f\rangle^{1 / 2}$ be the norm in $\mathcal{H}$, for $f$ and $g \in$ $\mathcal{H}$. The complex valued function $K(y, x)$ of $y$ and $x$ in $X$ is called a reproducing kernel of $\mathcal{H}$ if the followings are satisfied:

(i) For every $x, K_{x}(y)=K(y, x)$ as a function of $y$ belongs to $\mathcal{H}$.

(ii) The reproducing property: for every $x \in X$ and every $f \in \mathcal{H}$,

$$
f(x)=\left\langle f, K_{x}\right\rangle .
$$

So applying (2.1) to the function $K_{x}$ at $y$, we get

$$
K_{x}(y)=\left\langle K_{x}, K_{y}\right\rangle, \text { for } x, y \in X,
$$

and by (i),

$$
K(y, x)=\left\langle K_{x}, K_{y}\right\rangle, \text { for } x, y \in X .
$$

By the above relations, for $x \in X$ we obtain $\left\|K_{x}\right\|=\left\langle K_{x}, K_{x}\right\rangle^{1 / 2}=K(x, x)^{1 / 2}$. 
Definition 2.1.2. A Hilbert space $\mathcal{H}$ of functions on a set $X$ is called a reproducing kernel Hilbert space (sometimes abbreviated by RKHS) if there exists a reproducing kernel $K$ of $\mathcal{H}$, cf. Defintion 2.1.1.

The Hilbert space with reproducing kernel $K$ is denoted by $\mathcal{H}_{K}(X)$. Correspondingly norm will be denoted by $\|\cdot\|_{K}$ (or sometimes by $\|\cdot\|_{\mathcal{H}_{K}}$ ) and inner product will be denoted by $\langle\cdot, \cdot\rangle_{K}$ (or sometimes by $\langle\cdot, \cdot\rangle_{\mathcal{H}_{K}}$ ), if there is a need of distinction.

Theorem 2.1.3. If a Hilbert space $\mathcal{H}$ of functions on a set $X$ admits a reproducing kernel, then the reproducing kernel $K(y, x)$ is uniquely determined by the Hilbert space $\mathcal{H}$.

Proof. Let $K(y, x)$ be a reproducing kernel of $\mathcal{H}$. Suppose that there exists another kernel $K^{\prime}(y, x)$ of $\mathcal{H}$. Then, for all $x \in X$, applying (ii) for $K$ and $K^{\prime}$ we get

$$
\begin{aligned}
\left\|K_{x}-K_{x}^{\prime}\right\|^{2} & =\left\langle K_{x}-K_{x}^{\prime}, K_{x}-K_{x}^{\prime}\right\rangle \\
& =\left\langle K_{x}-K_{x}^{\prime}, K_{x}\right\rangle-\left\langle K_{x}-K_{x}^{\prime}, K_{x}^{\prime}\right\rangle \\
& =\left(K_{x}-K_{x}^{\prime}\right)(x)-\left(K_{x}-K_{x}^{\prime}\right)(x) \\
& =0
\end{aligned}
$$

Hence $K_{x}=K_{x}^{\prime}$, that is, $K_{x}(y)=K_{x}^{\prime}(y)$ for all $y \in X$. This means that $K(x, y)=K^{\prime}(x, y)$ for all $x, y \in X$.

Theorem 2.1.4. For a Hilbert space $\mathcal{H}$ of functions on $X$, there exists a reproducing kernel $K$ for $\mathcal{H}$ if and only if for every $x$ of $X$, the evaluation linear functional $\mathcal{H} \ni f \longmapsto f(x)$ is a bounded linear functional on $\mathcal{H}$.

Proof. Suppose that $K$ is the reproducing kernel for $\mathcal{H}$. By reproducing property and Schwarz inequality of the scalar product, for all $x \in X$,

$$
|f(x)|=\left|\left\langle f, K_{x}\right\rangle\right| \leq\|f\|\left\|K_{x}\right\|=\|f\|\left\langle K_{x}, K_{x}\right\rangle^{1 / 2}=\|f\| K(x, x)^{1 / 2}
$$

that is, the evaluation at $x$ is a bounded linear functional on $\mathcal{H}$. 
Conversely, if for all $x \in X$ the evaluation $\mathcal{H} \ni f \mapsto f(x)$ is a bounded linear functional on $\mathcal{H}$, then by the Riesz Representation Theorem, for all $x \in X$, there exists a function $g_{x}$ belonging to $\mathcal{H}$ such that

$$
f(x)=\left\langle f, g_{x}\right\rangle .
$$

If we put $K_{x}$ instead of $g_{x}$, then for all $y \in X$, we get $K_{x}(y)=g_{x}(y)$. Hence $K$ is a reproducing kernel for $\mathcal{H}$.

Definition 2.1.5. Let $X$ be an arbitrary set and $K$ be a kernel on $X$, that is, $K: X \times X \rightarrow \mathbb{C}$. The kernel $K$ is called Hermitian if for any finite set of points $\left\{y_{1}, \ldots, y_{n}\right\} \subseteq X$ and any complex numbers $\epsilon_{1}, \ldots, \epsilon_{n}$ we have

$$
\sum_{i, j=1}^{n} \bar{\epsilon}_{j} \epsilon_{i} K\left(y_{j}, y_{i}\right) \in \mathbb{R}
$$

and $K$ is called positive definite if

$$
\sum_{i, j=1}^{n} \bar{\epsilon}_{j} \epsilon_{i} K\left(y_{j}, y_{i}\right) \geq 0 .
$$

Equivalently, the last inequality means that for any finitely supported family of complex numbers $\left\{\epsilon_{x}\right\}_{x \in X}$ we have

$$
\sum_{x, y \in X} \bar{\epsilon}_{y} \epsilon_{x} K(y, x) \geq 0 .
$$

In brief, sometimes we will denote this by $[K(y, x)] \geq 0$ on $X$, or equivalently, we will say that $K$ is a positive definite matrix in the sense of $E$. H. Moore.

Theorem 2.1.6. The reproducing kernel $K(y, x)$ of a reproducing kernel Hilbert space $\mathcal{H}$ is a positive matrix in the sense of $E$. H. Moore.

Proof. We have

$$
\begin{aligned}
0 & \leq\left\|\sum_{i=1}^{n} \varepsilon_{i} K_{y_{i}}\right\|^{2}=\left\langle\sum_{i=1}^{n} \varepsilon_{i} K_{y_{i}}, \sum_{j=1}^{n} \varepsilon_{j} K_{y_{j}}\right\rangle \\
& =\sum_{i=1}^{n} \sum_{j=1}^{n} \varepsilon_{i} \overline{\varepsilon_{j}}\left\langle K_{y_{i}}, K_{y_{j}}\right\rangle=\sum_{i=1}^{n} \sum_{j=1}^{n} \varepsilon_{i} \overline{\varepsilon_{j}} K\left(y_{j}, y_{i}\right) .
\end{aligned}
$$

Hence,

$$
\sum_{i, j=1}^{n} K\left(y_{j}, y_{i}\right) \overline{\varepsilon_{j}} \varepsilon_{i} \geq 0 .
$$


Remark 2.1.7. Given a reproducing kernel Hilbert space $\mathcal{H}$ and its kernel $K(y, x)$ on $X$, then for all $x, y \in X$ we have the followings:

(i) $K(y, y) \geq 0$.

(ii) $K(y, x)=\overline{K(x, y)}$.

(iii) $|K(y, x)|^{2} \leq K(y, y) K(x, x)$, (Schwarz Inequality).

(iv) Let $x_{0} \in X$. Then the followings are equivalent:

(a) $K\left(x_{0}, x_{0}\right)=0$.

(b) $K\left(y, x_{0}\right)=0$ for all $y \in X$.

(c) $f\left(x_{0}\right)=0$ for all $f \in \mathcal{H}$.

Indeed, (i) and (ii) can be easily seen. For (iii), we use the Schwarz Inequality in $\mathcal{H}$ and get

$$
\begin{aligned}
|K(y, x)|^{2} & =\left|\left\langle K_{x}, K_{y}\right\rangle\right|^{2} \leq\left\|K_{x}\right\|\left\|K_{y}\right\|\left\|K_{x}\right\|\left\|K_{y}\right\|=\left\|K_{x}\right\|^{2}\left\|K_{y}\right\|^{2} \\
& =\left\langle K_{x}, K_{x}\right\rangle\left\langle K_{y}, K_{y}\right\rangle=K(x, x) K(y, y)
\end{aligned}
$$

which is the desired result.

As for (iv), it follows by (iii) that $K\left(x_{0}, x_{0}\right)=0$ is equivalent with $K\left(y, x_{0}\right)=0$ for all $y \in X$. Further, by the reproducing property, $K\left(y, x_{0}\right)=0$ for all $y \in X$ if and only if $f\left(x_{0}\right)=0$, for all $f$.

The following theorem can be regarded as a converse of Theorem 2.1.3.

Theorem 2.1.8. For any positive definite kernel $K(y, x)$ on $X$, there exists a uniquely determined Hilbert space $\mathcal{H}_{K}$ of functions on $X$, admitting the reproducing kernel $K(y, x)$.

Proof. We denote by $\mathcal{H}_{0}$ the space of all functions $f$ on $X$ such that there exists a finite set of points $x_{1}, x_{2}, \ldots, x_{n}$ of $X$ and complex numbers $\varepsilon_{1}, \varepsilon_{2}, \ldots, \varepsilon_{n}$,

$$
f(y)=\sum_{i=1}^{n} \varepsilon_{i} K\left(y, x_{i}\right),
$$


for all $y \in X$. Let $g(\cdot)=\sum_{i=1}^{m} \mu_{j} K\left(\cdot, y_{j}\right)$ be in $\mathcal{H}_{0}$. Define the inner product of the functions $f$ and $g$ from $\mathcal{H}_{0}$ by

$$
\langle f, g\rangle_{\mathcal{H}_{0}}=\sum_{i=1}^{n} \sum_{j=1}^{m} \varepsilon_{i} \bar{\mu}_{j}\left\langle K\left(\cdot, x_{i}\right), K\left(\cdot, y_{j}\right)\right\rangle_{\mathcal{H}_{0}}=\sum_{i=1}^{n} \sum_{j=1}^{m} \varepsilon_{i} \bar{\mu}_{j} K\left(y_{j}, x_{i}\right) .
$$

Then,

$$
\langle f, K(\cdot, x)\rangle_{\mathcal{H}_{0}}=\sum_{i=1}^{n} \epsilon_{i}\left\langle K\left(\cdot, x_{i}\right), K(\cdot, x)\right\rangle=\sum_{i=1}^{n} \epsilon_{i} K\left(x, x_{i}\right)=f(x)
$$

for all $x \in X$, that is, $\mathcal{H}_{0}$ has the reproducing property. This implies that the definition of the inner product in (2.3) does not depend on the representations of the functions $f$ and $g$ in $\mathcal{H}_{0}$. Moreover, it is easy to see that $\langle\cdot, \cdot\rangle_{\mathcal{H}_{0}}$ is linear in the first variable and Hermitian. Since $K$ is positive definite it follows that $\langle f, f\rangle_{\mathcal{H}_{0}} \geq 0$ for all $f \in \mathcal{H}_{0}$, hence we have the Schwarz Inequality for $\langle\cdot, \cdot\rangle_{\mathcal{H}_{0}}$. In addition, if $\langle f, f\rangle_{\mathcal{H}_{0}}=0,\|f\|=0$ and then by (2.4) for all $x \in X$,

$$
|f(x)| \leq\|f\|\|\mid K(\cdot, x)\|=0,
$$

which implies that $f \equiv 0$. Thus, $\left(\mathcal{H}_{0},\langle\cdot, \cdot\rangle_{\mathcal{H}_{0}}\right)$ is a pre-Hilbert space.

Now denote by $\mathcal{H}$ abstract the completion of $\mathcal{H}_{0}$ to a Hilbert space. We will show that $\mathcal{H}$ has a unique representation as a Hilbert space with reproducing kernel $K(y, x)$. Consider first any Cauchy sequence $\left(f_{n}\right)_{n \geq 1}$ in $\mathcal{H}_{0}$. Then for any $x \in X$ we have

$$
\begin{aligned}
\left|f_{m}(x)-f_{n}(x)\right| & =\left|\left\langle f_{m}, K_{x}\right\rangle_{\mathcal{H}_{0}}-\left\langle f_{n}, K_{x}\right\rangle_{\mathcal{H}_{0}}\right| \\
& =\left|\left\langle f_{m}-f_{n}, K_{x}\right\rangle_{\mathcal{H}_{0}}\right| \\
& \leq\left\|f_{m}-f_{n}\right\|_{\mathcal{H}_{0}} K(x, x)^{1 / 2} .
\end{aligned}
$$

So, there exists the function $f: X \rightarrow \mathbb{C}$ such that for all $x \in X$,

$$
\lim _{n \rightarrow \infty} f_{n}(x)=f(x)
$$

Moreover, we have

$$
\|f\|_{\mathcal{H}}=\lim _{n \rightarrow \infty}\left\|f_{n}\right\|_{\mathcal{H}_{0}}
$$


and for any two Cauchy sequences $\left(f_{n}\right)$ and $\left(g_{n}\right)$ in $\mathcal{H}_{0}$, denoting by $f$ and, respectively $g$, the corresponding pointwise limit of $\left(f_{n}\right)$ and $\left(g_{n}\right)$, we have

$$
\langle f, g\rangle_{\mathcal{H}}=\lim _{n, m \rightarrow \infty}\left\langle f_{n}, g_{m}\right\rangle_{\mathcal{H}_{0}} .
$$

We can easily see that, for any two Cauchy sequences $\left(f_{n}\right)$ and $\left(g_{n}\right)$, these limits exist and are independent of the approximating sequences $\left(f_{n}\right)$ and $\left(g_{n}\right)$ of the limits $f$ and $g$, respectively.

Let us note that (2.5) yields a concrete representation of $\mathcal{H}$ as a space of functions on $X$. In addition, $K$ has the reproducing property with respect to $\mathcal{H}$. To see this, let $f \in \mathcal{H}$ and $\left(f_{n}\right) \subset \mathcal{H}$ such that $f_{n} \rightarrow f$ as $n \rightarrow \infty$ strongly. Then for all $x \in X$,

$$
\begin{aligned}
f(x) & =\lim _{n \rightarrow \infty} f_{n}(x)=\lim _{n \rightarrow \infty}\left\langle f_{n}, K_{x}\right\rangle_{\mathcal{H}_{0}} \\
& =\left\langle\lim _{n \rightarrow \infty} f_{n}, K_{x}\right\rangle_{\mathcal{H}_{0}}=\left\langle f, K_{x}\right\rangle_{\mathcal{H}}
\end{aligned}
$$

It remains to show the uniqueness of the Hilbert space $\mathcal{H}$ admitting the reproducing kernel $K$. Suppose $\mathcal{H}_{1}$ is another Hilbert space with the same reproducing kernel $K$. By definition, for any $x \in X, K_{x} \in \mathcal{H}_{1}$ and then we have $\mathcal{H}_{0} \subseteq \mathcal{H}_{1}$. Also, for any $f, g \in \mathcal{H}_{0}$, because of the reproducing property we have

$$
\langle f, g\rangle_{\mathcal{H}_{0}}=\langle f, g\rangle_{\mathcal{H}_{1}} .
$$

If $f \in \mathcal{H}_{1}$ such that $0=\left\langle f, K_{x}\right\rangle_{\mathcal{H}_{1}}=f(x)$ for all $x \in X$, then $f \equiv 0$. Thus, the family $\left\{K_{x}: x \in X\right\}$ is total in $\mathcal{H}_{1}$. So for any $f \in \mathcal{H}_{1}$, we can take a Cauchy sequence $\left(f_{n}\right)_{n \geq 1}$ in $\mathcal{H}_{0}$ such that $\lim _{n \rightarrow \infty} f_{n}=f$. Hence, (2.6) is valid in $\mathcal{H}_{0}$.

Now since we have $\mathcal{H}_{0} \subseteq \mathcal{H}_{1}$ and (2.6), we obtain $\mathcal{H} \subseteq \mathcal{H}_{1}$. Also from the construction of $\mathcal{H}$, we get $\mathcal{H}_{1} \subseteq \mathcal{H}$. Thus, we have $\mathcal{H}_{1}=\mathcal{H}$.

Finally, we have to show that the inner products and the norms are equal in $\mathcal{H}$ and $\mathcal{H}_{1}$. Consider any $f, g \in \mathcal{H}_{1}$ and any Cauchy sequences $\left(f_{n}\right)_{n \geq 1}$ and $\left(g_{n}\right)_{n \geq 1}$ in $\mathcal{H}_{0}$ which converge to $f$ and $g$ respectively. We have

$$
\langle f, g\rangle_{\mathcal{H}_{1}}=\lim _{n \rightarrow \infty}\left\langle f_{n}, g_{n}\right\rangle_{\mathcal{H}_{1}}=\lim _{n \rightarrow \infty}\left\langle f_{n}, g_{n}\right\rangle_{\mathcal{H}_{0}}=\langle f, g\rangle_{\mathcal{H}}
$$

and hence the norms in $\mathcal{H}$ and $\mathcal{H}_{1}$ are equal. 
Theorem 2.1.9. Every sequence of functions $\left(f_{n}\right)_{n \geq 1}$ which converges strongly to a function $f$ in $\mathcal{H}_{K}(X)$, converges also in the pointwise sense, that is, $\lim _{n \rightarrow \infty} f_{n}(x)=f(x)$, for any point $x \in X$. Further, this convergence is uniform on every subset of $X$ on which $x \longmapsto K(x, x)$ is bounded.

Proof. For $x \in X$, using the reproducing property and the Schwartz Inequality,

$$
\begin{aligned}
\left|f(x)-f_{n}(x)\right| & =\left|\left\langle f, K_{x}\right\rangle-\left\langle f_{n}, K_{x}\right\rangle\right| \\
& =\left|\left\langle f-f_{n}, K_{x}\right\rangle\right| \mid \\
& \leq\left\|f-f_{n}\right\| \cdot\left\|K_{x}\right\| \\
& =\left\|f-f_{n}\right\| \cdot K(x, x)^{1 / 2} .
\end{aligned}
$$

Therefore, $\lim _{n \rightarrow \infty} f_{n}(x)=f(x)$, for any point $x \in X$.

Moreover, it is clear from the above inequality that this convergence is uniform on every subset of $X$ on which $x \longmapsto K(x, x)$ is bounded.

In the following we will use the following notation: given $X$ an abstract nonempty set and $H$ and $K$ two Hermitian kernels on $X$, we denote

$$
[H(y, x)] \leq[K(y, x)] \text { on } X
$$

whenever for any natural number $n$, any finite set $\left\{x_{1}, \ldots, x_{n}\right\} \subseteq X$ and any complex numbers $\epsilon_{1}, \ldots, \epsilon_{n}$ we have

$$
\sum_{i, j=1}^{n} \bar{\epsilon}_{j} \epsilon_{i} H\left(x_{j}, x_{i}\right) \leq \sum_{i, j=1}^{n} \bar{\epsilon}_{j} \epsilon_{i} K\left(x_{j}, x_{i}\right)
$$

Theorem 2.1.10. A complex valued function $g$ on $X$ belongs to the reproducing kernel Hilbert space $\mathcal{H}_{K}(X)$ if and only if there exists $0 \leq \gamma<\infty$ such that,

$$
[g(y) \overline{g(x)}] \leq \gamma^{2}[K(y, x)] \text { on } X .
$$

The minimum of all such $\gamma$ coincides with $\|g\|$.

Proof. By the reproducing property, $g \in \mathcal{H}_{K}$ and $\|g\| \leq \gamma$ is equivalent with the existence of $f \in \mathcal{H}_{K}(X)$ such that $\|f\| \leq \gamma$ and $g(x)=\left\langle f, K_{x}\right\rangle$ for $x \in X$. By 
applying the Abstract Interpolation Theorem (see Theorem A.2.6) we obtain the inequality (2.9). The converse implication is also a consequence of the Abstract Interpolation Theorem.

Theorem 2.1.11. Let $K^{(1)}(y, x)$ and $K^{(2)}(y, x)$ be two positive definite kernels on $X$. Then the following assertions are mutually equivalent:

(i) $\mathcal{H}_{K^{(1)}}(X) \subseteq \mathcal{H}_{K^{(2)}}(X)$, (set inclusion).

(ii) There exists $0 \leq \gamma<\infty$ such that

$$
\left[K^{(1)}(y, x)\right] \leq \gamma^{2}\left[K^{(2)}(y, x)\right]
$$

If this is the case, the inclusion map $J$ in (i) is continuous, and its norm is given by the minimum of $\gamma$ in (ii).

Proof. Denote the norm and the inner product in $\mathcal{H}_{K^{(i)}}(X)$ by $\|\cdot\|_{i}$ and $\langle\cdot, \cdot\rangle_{i}$, respectively.

Let (i) be satisfied. Set $J: \mathcal{H}_{K^{(1)}}(X) \longrightarrow \mathcal{H}_{K^{(2)}}(X)$, the inclusion map.

Claim: $J$ is a closed and continuous operator.

Suppose that $f_{n} \rightarrow g$ in $\mathcal{H}_{K^{(1)}}(X)$ and $f_{n} \rightarrow h$ in $\mathcal{H}_{K^{(2)}}(X)$. As point evaluations are continuous in $\mathcal{H}_{K^{(i)}}(X),(i=1,2)$, we get

$$
f_{n}(x) \rightarrow g(x) \text { and } f_{n}(x) \rightarrow h(x)
$$

which implies that $g(x)=h(x)$ for all $x$, since the limit is unique. So $J$ is closed. Since $J$ is closed, we know that by the Closed Graph Theorem any closed linear operator between Hilbert spaces is continuous. Hence $J$ is continuous, as claimed.

Now, for all $f \in \mathcal{H}_{K^{(1)}}(X)$ and for all $x \in X$, by reproducing property we have $f(x)=\left\langle f, K_{x}^{(1)}\right\rangle_{1}$ and $(J f)(x)=\left\langle J f, K_{x}^{(2)}\right\rangle_{2}$. Then by using this and the inclusion property of $J$, for all $x \in X$, we have

$$
\left\langle f, J^{*} K_{x}^{(2)}\right\rangle_{1}=\left\langle J f, K_{x}^{(2)}\right\rangle_{2}=(J f)(x)=\left\langle f, K_{x}^{(1)}\right\rangle_{1}
$$


and hence we obtain $J^{*} K_{x}^{(2)}=K_{x}^{(1)}$ for all $x \in X$.

Finally, for any $\gamma \geq\|J\|$ and any finitely supported family of complex numbers $\left\{\epsilon_{x}\right\}_{x \in X}$, we have

$$
\begin{aligned}
\sum_{x, y} \epsilon_{x} \overline{\epsilon_{y}} K^{(1)}(y, x) & =\left\langle\sum_{x} \epsilon_{x} K_{x}^{(1)}, \sum_{y} \epsilon_{y} K_{y}^{(1)}\right\rangle=\left\|\sum_{x} \epsilon_{x} K_{x}^{(1)}\right\|_{1}^{2} \\
& =\left\|J^{*}\left(\sum_{x} \epsilon_{x} K_{x}^{(2)}\right)\right\|_{1}^{2} \leq \gamma^{2}\left\|\sum_{x} \epsilon_{x} K_{x}^{(2)}\right\|^{2} \\
& =\gamma^{2}\left\langle\sum_{x} \epsilon_{x} K_{x}^{(2)}, \sum_{y} \epsilon_{y} K_{y}^{(2)}\right\rangle \\
& =\gamma^{2} \sum_{x, y} \epsilon_{x} \overline{\epsilon_{y}} K^{(2)}(y, x)
\end{aligned}
$$

Hence,

$$
\left[K^{(1)}(y, x)\right] \leq \gamma^{2}\left[K^{(2)}(y, x)\right] .
$$

Conversely, suppose that (ii) is satisfied for some $0 \leq \gamma<\infty$. This means that for any finitely supported family of complex numbers $\left\{\epsilon_{x}\right\}_{x \in X}$, that is denoted by $\left[\epsilon_{x}\right]$,

$$
\sum_{x, y} \epsilon_{x} \overline{\epsilon_{y}} K^{(1)}(y, x) \leq \gamma^{2} \sum_{x, y} \epsilon_{x} \overline{\epsilon_{y}} K^{(2)}(y, x) .
$$

Taking the minimum of $\gamma$ in Theorem 2.1.10, we have the norm of any function $f$ on $X$ given by

$$
\|f\|_{i}^{2}=\sup _{\left[\epsilon_{x}\right]} \frac{\left|\sum_{x} \epsilon_{x} f(x)\right|^{2}}{\sum_{x, y} \epsilon_{x} \overline{\epsilon_{y}} K^{(i)}(y, x)}, \quad(i=1,2),
$$

with $\|f\|_{i}=\infty$ if $f$ is not in $\mathcal{H}_{K^{(i)}}(X)$. Now since $\left\{K_{x}^{(i)}: x \in X\right\}$ is total in $\mathcal{H}_{K^{(i)}}(X),(i=1,2)$ and using the Schwarz Inequality for the norms $\|f\|_{1}$ and $\|f\|_{2}$, we get

$$
\|f\|_{2} \leq \gamma\|f\|_{1} \text { for } f \in \mathcal{H}_{K^{(1)}}(X) .
$$

Hence, $\mathcal{H}_{K^{(1)}}(X) \subseteq \mathcal{H}_{K^{(2)}}(X)$ with $\|J\| \leq \gamma$.

Suppose that there is a map $\phi$ from a set $X$ to a Hilbert space $\mathcal{H}$ such that $x \longmapsto \phi_{x}$. Then $\phi$ can be used to define a positive definite kernel

$$
K(y, x)=\left\langle\phi_{x}, \phi_{y}\right\rangle \text { for } x, y \in X .
$$


Theorem 2.1.12. Let $\phi: X \longmapsto \mathcal{H}$ and $K$ be defined as in (2.10). Let $T$ be the linear operator from $\mathcal{H}$ to the space of functions on $X$, defined by

$$
(T f)(x)=\left\langle f, \phi_{x}\right\rangle \text { for } x \in X, f \in \mathcal{H} .
$$

Then $\operatorname{Ran}(T)$ coincides with $\mathcal{H}_{K}(X)$ and

$$
\|T f\|_{K}=\left\|P_{\mathcal{M}} f\right\| \text { for } f \in \mathcal{H},
$$

where $\mathcal{M}$ is the orthogonal complement of $\operatorname{ker}(T), P_{\mathcal{M}}$ is the orthogonal projection onto $\mathcal{M}$ and $\|\cdot\|_{K}$ denotes the norm in $\mathcal{H}_{K}(X)$.

Proof. To see the positive definiteness of $K(y, x)$, let $X \ni x \longmapsto \varepsilon_{x}$ be a complex valued function with finite support. Then,

$$
\begin{aligned}
\sum_{x, y} \overline{\varepsilon_{y}} \varepsilon_{x} K(y, x) & =\sum_{x, y} \overline{\varepsilon_{y}} \varepsilon_{x}\left\langle\phi_{x}, \phi_{y}\right\rangle=\sum_{x, y}\left\langle\varepsilon_{x} \phi_{x}, \varepsilon_{y} \phi_{y}\right\rangle \\
& =\left\langle\sum_{x} \varepsilon_{x} \phi_{x}, \sum_{y} \varepsilon_{y} \phi_{y}\right\rangle=\left\|\sum_{x} \varepsilon_{x} \phi_{x}\right\|^{2} \geq 0 \text { for } x, y \in X .
\end{aligned}
$$

Hence $K(y, x)$ is positive definite.

Let $x \in X$ and $K_{x}: X \longrightarrow \mathbb{C}$. For all $y \in X, K_{x}(y)=\left\langle\phi_{x}, \phi_{y}\right\rangle=\left(T \phi_{x}\right)(y)$. So, $\operatorname{Ran}(T)$ contains all the functions $K_{x}, x \in X$, where $K_{x}(y)=K(y, x)=\left\langle\phi_{x}, \phi_{y}\right\rangle$, $y \in X$. Since $\operatorname{Ran}(T)$ is a linear space, then linear span of $\left\{K_{x}: x \in X\right\}$, that is, $\operatorname{lin}\left\{K_{x}: x \in X\right\}=\mathcal{H}_{0}$, will be in $\operatorname{Ran}(T)$, i.e. $\mathcal{H}_{0} \subseteq \operatorname{Ran}(T)$.

Claim: $T: \operatorname{lin}\left\{\phi_{x}: x \in X\right\} \longrightarrow \mathcal{H}_{0}$ is isometric.

Since $T \phi_{x}=K_{x}$, for all $x \in X$, then $T\left(\sum_{x} \varepsilon_{x} \phi_{x}\right)=\sum_{x} \varepsilon_{x} K_{x}$. Hence,

$$
\begin{aligned}
\left\langle T\left(\sum_{x} \varepsilon_{x} \phi_{x}\right), T\left(\sum_{y} \eta_{y} \phi_{y}\right)\right\rangle_{K} & =\left\langle\sum_{x} \varepsilon_{x} K_{x}, \sum_{y} \eta_{y} K_{y}\right\rangle_{K}=\sum_{x, y} \overline{\eta_{y}} \varepsilon_{x} K(y, x) \\
& =\sum_{x, y} \overline{\eta_{y}} \varepsilon_{x}\left\langle\phi_{x}, \phi_{y}\right\rangle_{\mathcal{H}}=\left\langle\sum_{x} \varepsilon_{x} \phi_{x}, \sum_{y} \eta_{y} \phi_{y}\right\rangle_{\mathcal{H}} .
\end{aligned}
$$

That is, $T \operatorname{lin}\left\{\phi_{x}: x \in X\right\} \longrightarrow \operatorname{lin}\left\{K_{x}: x \in X\right\}=\mathcal{H}_{0}$ is isometric. Clearly, $T\left(\operatorname{lin}\left\{\phi_{x}: x \in X\right\}\right)=\mathcal{H}_{0}$. 
Now take $f$ in $\operatorname{ker}(T)$. So $T f=0$, i.e. $(T f)(x)=0$ for all $x \in X$. But $(T f)(x)=\left\langle f, \phi_{x}\right\rangle=0$ for all $x \in X$ and $\mathrm{T}$ is linear which implies

$$
f \perp \operatorname{lin}\left\{\phi_{x}: x \in X\right\} .
$$

If $f \in \operatorname{lin}\left\{\phi_{x}: x \in X\right\}^{\perp}=\left\{\phi_{x}: x \in X\right\}^{\perp}$, then for all $x \in X$,

$$
0=\left\langle f, \phi_{x}\right\rangle=(T f)(x) .
$$

That is, $T f=0$ and $\operatorname{ker}(T)=\operatorname{lin}\left\{\phi_{x}: x \in X\right\}^{\perp}$. By this, we reach

$$
\operatorname{ker}(T)^{\perp}=\operatorname{lin}\left\{\phi_{x}: x \in X\right\}^{\perp^{\perp}}=\overline{\operatorname{lin}\left\{\phi_{x}: x \in X\right\}}=: \mathcal{M} .
$$

As $\mathcal{M}$ is a closed subspace, then $\mathcal{H}$ can be written as $\mathcal{H}=\mathcal{M} \oplus \mathcal{M}^{\perp}$. Since

$$
T: \operatorname{lin}\left\{\phi_{x}: x \in X\right\} \longrightarrow \mathcal{H}_{0} \subseteq \mathcal{H}_{K}(X)
$$

is isometric and surjective and since $\mathcal{H}_{0}$ is dense in $\mathcal{H}_{K}(X)$, it follows that $T\left(\overline{\operatorname{lin}\left\{\phi_{x}: x \in X\right\}}\right) \longrightarrow \overline{\mathcal{H}_{0}}=\mathcal{H}_{K}(X)$. Hence, $T M=\mathcal{H}_{K}(X)=T\left(\mathcal{M} \oplus \mathcal{M}^{\perp}\right)=$ $T \mathcal{H}=\operatorname{Ran}(T)$.

Finally, to see the equality of norms, take $f \in \mathcal{H}=\mathcal{M} \oplus \mathcal{M}^{\perp}$. It can be written as $f=P_{\mathcal{M}} f+\left(I-P_{\mathcal{M}}\right) f$, where $I-P_{\mathcal{M}}=P_{k e r T}$. Then, since $T$ is isometric on $\mathcal{M}$,

$$
\|T f\|_{K}=\left\|T\left(P_{M} f+P_{k e r T} f\right)\right\|_{K}=\left\|T P_{M} f\right\|_{K}=\left\|P_{M} f\right\|_{K} .
$$

The next result that concludes this section shows that the assumptions in (2.10) is by no means restrictive, if we consider positive definite kernels.

Theorem 2.1.13. (Kolmogorov Decomposition) Let $K(y, x)$ be a positive definite kernel on an abstract set $X$. Then there exists a Hilbert space $\mathcal{H}$ and a function $\phi: X \rightarrow \mathcal{H}$ such that

$$
K(y, x)=\left\langle\phi_{x}, \phi_{y}\right\rangle \text { for } x, y \in X .
$$

In addition, the Hilbert space $\mathcal{H}$ can be chosen in such a way that the set $\left\{\phi_{x}\right\}_{x \in X}$ is total in $\mathcal{H}$ and in this case the pair $(\phi, \mathcal{H})$ is unique in the following sense: for any other pair $(\psi, \mathcal{K})$, where $\psi: X \rightarrow \mathcal{K}$ and $\mathcal{K}$ is a Hilbert space such that $\left\{\psi_{x}\right\}_{x \in X}$ is total in $\mathcal{K}$ and $K(y, x)=\left\langle\psi_{x}, \psi_{y}\right\rangle_{\mathcal{K}}$ for all $x, y \in X$, there exists a unitary operator $U \in \mathcal{L}(\mathcal{H}, \mathcal{K})$ such that $U \phi_{x}=\psi_{x}$ for all $x \in X$. 
Proof. Since $K$ is positive definite, by Theorem 2.1.8 there exists the reproducing kernel space $\mathcal{H}_{K}$ with reproducing kernel $K$. Let $\phi_{x}=K_{x} \in \mathcal{H}_{K}$ for all $x \in X$. By the reproducing property, for all $x, y \in X$ we have

$$
K(y, x)=\left\langle K_{x}, K_{y}\right\rangle_{\mathcal{H}_{K}}
$$

and $\left\{K_{x}\right\}_{x \in X}$ is a total subset of $\mathcal{H}_{K}$.

To prove uniqueness, let $(\psi, \mathcal{K})$ be a pair as in the statement and define $U \phi_{x}=\psi_{x}$ for all $x \in X$. Clearly $U$ extends by linearity as a linear mapping $U: \operatorname{lin}\left\{\phi_{x}: x \in X\right\} \rightarrow \operatorname{lin}\left\{\psi_{x}: x \in X\right\}$. In addition, for any finitely supported families of complex numbers $\left\{\epsilon_{x}\right\}_{x \in X}$ and $\left\{\eta_{y}\right\}_{y \in X}$ we have

$$
\begin{aligned}
\left\langle U\left(\sum_{x \in X} \epsilon_{x} \phi_{x}\right), U\left(\sum_{y \in X} \eta_{y} \phi_{y}\right)\right\rangle_{\mathcal{K}} & =\left\langle\left(\sum_{x \in X} \epsilon_{x} \psi_{x}\right),\left(\sum_{y \in X} \eta_{y} \psi_{y}\right)\right\rangle_{\mathcal{K}} \\
& =\sum_{x, y \in X} \bar{\epsilon}_{y} \epsilon_{x}\left\langle\psi_{x}, \psi_{y}\right\rangle_{\mathcal{K}} \\
& =\sum_{x, y \in X} \bar{\epsilon}_{y} \epsilon_{x} K(y, x)=\sum_{x, y \in X} \bar{\epsilon}_{y} \epsilon_{x}\left\langle\phi_{x}, \phi_{y}\right\rangle_{\mathcal{H}_{K}} \\
& =\left\langle\left(\sum_{x \in X} \epsilon_{x} \phi_{x}\right),\left(\sum_{y \in X} \eta_{y} \phi_{y}\right)\right\rangle_{\mathcal{H}_{K}}
\end{aligned}
$$

which shows that $U$ is isometric. Due to the fact that both families $\left\{\phi_{x}\right\}_{x \in X}$ and $\left\{\psi_{y}\right\}_{y \in X}$ are total in $\mathcal{H}_{K}$ and, respectively, $\mathcal{K}$, it follows that $U$ can be uniquely extended to a unitary operator $U: \mathcal{H}_{K} \rightarrow \mathcal{K}$. By definition, $U$ satisfies the condition $U \phi_{x}=\psi_{x}$ for all $x \in X$.

\subsection{Operations with Reproducing Kernel Hilbert Spaces}

Let $K(y, x)$ be a positive definite kernel on $X$ and $\mathcal{H}=\mathcal{H}_{K}(X)$ be the RKHS. Let $\mathcal{M}$ be a closed subspace of $\mathcal{H}_{K}(X)$. We know $\mathcal{M}$ is a Hilbert space since it is closed. As every point evaluation functional is continuous in $\mathcal{H}_{K}(X)$ and $\mathcal{M}$ is a closed subspace, then every point evaluation functional is continuous also in $\mathcal{M}$. Thus, $\mathcal{M}$ is a RKHS. 
Denote by $P_{\mathcal{M}}$ the orthogonal projection onto $\mathcal{M}$. This means that, for $h \in$ $\mathcal{H}_{K}(X), P_{\mathcal{M}}(h)=h_{\mathcal{M}} \in \mathcal{M}$ where $h=h_{\mathcal{M}}+h_{\mathcal{M}^{\perp}}$, with $h_{\mathcal{M}} \in \mathcal{M}, h_{\mathcal{M}^{\perp}} \in \mathcal{M}^{\perp}$. If $P_{\mathcal{M}} K_{x} \in \mathcal{M}$, we have $f(x)=\left\langle f, P_{\mathcal{M}} K_{x}\right\rangle$ for all $f \in \mathcal{M}$. Then we have the reproducing kernel $K^{\mathcal{M}}(y, x)$ for $\mathcal{M}$ as

$$
K^{\mathcal{M}}(y, x)=\left\langle P_{\mathcal{M}} K_{x}, P_{\mathcal{M}} K_{y}\right\rangle=\left\langle P_{\mathcal{M}} P_{\mathcal{M}}^{*} K_{x}, K_{y}\right\rangle=\left\langle P_{\mathcal{M}} K_{x}, K_{y}\right\rangle
$$

Let $K^{(0)}(y, x)$ be the restriction of $K(y, x)$ to a subset $X_{0}$ of $X$, i.e. $K^{(0)}(y, x)=$ $\left.K(y, x)\right|_{X_{0} \times X_{0}}$. Since $K(y, x)$ is positive definite, then so is $K^{(0)}(y, x)$. Then by Theorem 2.1.8 there exists a unique RKHS admitting $K^{(0)}(y, x)$ as its reproducing kernel. Denote this RKHS by $\mathcal{H}_{K^{(0)}}(X)$. The following theorem gives the relation between $\mathcal{H}_{K}(X)$ and $\mathcal{H}_{K^{(0)}}(X)$.

Theorem 2.2.1. Let $K^{(0)}(y, x)$ be the restriction of $K(y, x)$ to a subset $X_{0}$ of $X$, $\mathcal{H}_{K^{(0)}}(X)$ be the $R K H S$ admitting $K^{(0)}(y, x)$ as its reproducing kernel and $\mathcal{H}_{K}(X)$ be the RKHS with its reproducing kernel $K(y, x)$. Then

$$
\mathcal{H}_{K^{(0)}}\left(X_{0}\right)=\left\{\left.f\right|_{X_{0}}: f \in \mathcal{H}_{K}(X)\right\}
$$

and

$$
\|h\|_{K^{(0)}}=\min \left\{\|f\|_{K}:\left.f\right|_{X_{0}}=h\right\} \quad \text { for } h \in \mathcal{H}_{K^{(0)}}\left(X_{0}\right)
$$

Proof. For $x, y \in X_{0}$, we have

$$
K^{(0)}(y, x)=K(y, x) \text { and so }\left\langle K_{x}^{(0)}, K_{y}^{(0)}\right\rangle_{K^{(0)}}=\left\langle K_{x}, K_{y}\right\rangle_{K}
$$

Define a map $S$ such that $S K_{x}^{(0)}=K_{x}$ for all $x \in X_{0}$, which is uniquely extended to an isometry from the closed linear span of $\left\{K_{x}^{0}: x \in X_{0}\right\}$ that coincides with $\mathcal{H}_{K^{(0)}}\left(X_{0}\right)$ onto the closed linear span $\mathcal{M}$ of $\left\{K_{x}: x \in X_{0}\right\}$.

Let $T=S P_{\mathcal{M}}$ where $P_{\mathcal{M}}$ is the orthogonal projection to $\mathcal{M}$, i.e. $P_{\mathcal{M}}$ : $\mathcal{H}_{K}(X) \longrightarrow \mathcal{M}$. We have

$$
T: \mathcal{H}_{K}(X)=\mathcal{M} \oplus \mathcal{M}^{\perp} \longrightarrow \mathcal{H}_{K^{(0)}}\left(X_{0}\right) .
$$

Since

$$
\left(T K_{x}\right)(y)=K_{x}^{(0)}(y)=K_{x}(y) \text { for } x, y \in X_{0}
$$


then

$$
(T f)(y)=f(y) \text { for } f \in \mathcal{M} \text { and } y \in X_{0}
$$

and when $(T f)(y)=0,(T f)(y)=f(y)=\left\langle f, K_{y}\right\rangle=0$ for $f \in \mathcal{M}^{\perp}$ and $y \in X_{0}$. So $T$ is the restriction map to $X_{0}$ and

$$
(T f)(x)=\left\langle T f, K_{x}^{(0)}\right\rangle_{K^{(0)}}=\left\langle f, T^{*} K_{x}^{(0)}\right\rangle_{K}, \text { for } x \in X_{0}
$$

Hence we have $(T f)(x)=\left\langle f, \phi_{x}\right\rangle=\left\langle f, T^{*} K_{x}^{(0)}\right\rangle_{K}$ which gives us $\phi_{x}=T^{*} K_{x}^{(0)}$. By this with Theorem 2.1 .12 and taking into account that $T^{*}$ is isometric,

$$
\left\langle\phi_{x}, \phi_{y}\right\rangle_{K}=\left\langle T^{*} K_{x}^{(0)}, T^{*} K_{y}^{(0)}\right\rangle_{K}=\left\langle K_{x}^{(0)}, K_{y}^{(0)}\right\rangle_{K^{(0)}}=K^{(0)}(y, x)
$$

for all $x, y \in X_{0}$.

Let $K^{(1)}(y, x)$ and $K^{(2)}(y, x)$ be two positive definite kernels. Then

$$
K(y, x)=K^{(1)}(y, x)+K^{(2)}(y, x)
$$

is also a positive definite kernel.

Let $\mathcal{H}_{K^{(1)}}, \mathcal{H}_{K^{(2)}}$ and $\mathcal{H}_{K}$ be RKHSs with reproducing kernels $K^{(1)}(y, x), K^{(2)}(y, x)$ and $K(y, x)$, respectively, with $K=K^{(1)}+K^{(2)}$.

Theorem 2.2.2. Let $K^{(1)}(y, x)$ and $K^{(2)}(y, x)$ be two positive definite kernels and $K=K^{(1)}+K^{(2)}$. Then

$$
\mathcal{H}_{K}(X)=\mathcal{H}_{K^{(1)}}(X)+\mathcal{H}_{K^{(2)}}(X), \quad \text { (algebraic sum) }
$$

and for $f \in \mathcal{H}_{K^{(1)}}(X)$ and $g \in \mathcal{H}_{K^{(2)}}(X)$,

$$
\|f+g\|_{K}^{2}=\min \left\{\|f+h\|_{K^{(1)}}^{2}+\|g-h\|_{K^{(2)}}^{2}: h \in \mathcal{H}_{K^{(1)}}(X) \cap \mathcal{H}_{K^{(2)}}(X)\right\} .
$$

Proof. We have

$$
K(y, x)=K^{(1)}(y, x)+K^{(2)}(y, x)=\left\langle K_{x}^{(1)}, K_{y}^{(1)}\right\rangle_{K^{(1)}}+\left\langle K_{x}^{(2)}, K_{y}^{(2)}\right\rangle_{K^{(2)}} .
$$

Consider the direct sum Hilbert space $\mathcal{H}=\mathcal{H}_{K^{(1)}} \oplus \mathcal{H}_{K^{(2)}}$. Since both $\mathcal{H}_{K^{(1)}}$ and $\mathcal{H}_{K^{(2)}}$ are Hilbert spaces, so is $\mathcal{H}$. Then, by the definition of inner product for direct sum, we have

$$
\left\langle K_{x}, K_{y}\right\rangle_{K}=K(y, x)=K^{(1)}(y, x)+K^{(2)}(y, x)=\left\langle K_{x}^{(1)} \oplus K_{x}^{(2)}, K_{y}^{(1)} \oplus K_{y}^{(2)}\right\rangle_{K}
$$


Consider the map $\phi$ such that $\phi(x)=K_{x}^{(1)} \oplus K_{x}^{(2)}$. Then we have

$$
K(y, x)=\left\langle K_{x}^{(1)} \oplus K_{x}^{(2)}, K_{y}^{(1)} \oplus K_{y}^{(2)}\right\rangle_{K}=\left\langle\phi_{x}, \phi_{y}\right\rangle .
$$

Using the operator $T$ in Theorem 2.1.12, where $(T f)(x)=\left\langle f, \phi_{x}\right\rangle$, we get

$$
\begin{aligned}
(T(f \oplus g))(x) & =\left\langle f \oplus g, \phi_{x}\right\rangle=\left\langle f \oplus g, K_{x}^{(1)} \oplus K_{x}^{(2)}\right\rangle_{K} \\
& =\left\langle f, K_{x}^{(1)}\right\rangle_{K^{(1)}}+\left\langle g, K_{x}^{(2)}\right\rangle_{K^{(2)}} \\
& =f(x)+g(x) .
\end{aligned}
$$

So, $T(f \oplus g)=f+g$. This shows by Theorem 2.1.12 that $\mathcal{H}_{K}(X)=\operatorname{Ran}(T)=$ $\mathcal{H}_{K^{(1)}}(X)+\mathcal{H}_{K^{(2)}}(X)$. Again by the same theorem,

$$
\|f+g\|^{2}=\left\|P_{\mathcal{M}}(f \oplus g)\right\|^{2}, \text { where } \mathcal{M}=(\operatorname{ker}(T))^{\perp} .
$$

Next we show that

$$
\operatorname{ker}(T)=\left\{h \oplus(-h): h \in \mathcal{H}_{K^{(1)}}(X) \cap \mathcal{H}_{K^{(2)}}(X)\right\} .
$$

If $h \in \mathcal{H}_{K^{(1)}}(X) \cap \mathcal{H}_{K^{(2)}}(X)$, then $T(h \oplus(-h))=h-h=0$. Conversely, if $h_{1} \oplus h_{2} \in \operatorname{ker}(T)$, then $0=T\left(h_{1} \oplus h_{2}\right)=h_{1}+h_{2}$ implies that $h_{2}=-h_{1}$. Thus $h \in \mathcal{H}_{K^{(1)}}(X) \cap \mathcal{H}_{K^{(2)}}(X)$.

Then by Theorem 2.1.12 we have $\mathcal{M}=(\operatorname{ker}(T))^{\perp}$ which implies $\mathcal{M}^{\perp}=$ $\operatorname{ker}(T)$. So, $h \oplus(-h) \in \mathcal{M}^{\perp}$. Consider the quotient

$$
\mathcal{H} / \mathcal{M}^{\perp}:=\left\{\widetilde{h}+\mathcal{M}^{\perp}: \widetilde{h} \in \mathcal{H}\right\} .
$$

Let $f \in \mathcal{H}_{K^{(1)}}(X), g \in \mathcal{H}_{K^{(2)}}(X)$ and $f \oplus g \in \mathcal{H}=\mathcal{H}_{K^{(1)}} \oplus \mathcal{H}_{K^{(2)}}$. Then, for $\widehat{k} \in \mathcal{H} / \mathcal{M}^{\perp}$

$$
\widehat{k}=\left\{\widetilde{k}+\widetilde{h}: \widetilde{h} \in M^{\perp}\right\},
$$

where $\widetilde{k}=f \oplus g \in \mathcal{H}, \widetilde{h}=h \oplus(-h) \in \mathcal{M}^{\perp}$. Then,

$$
\widehat{h}=\left\{f \oplus g+h \oplus(-h): f \oplus g \in \mathcal{H}, \quad h \oplus(-h) \in \mathcal{M}^{\perp}\right\} .
$$

Taking the norm of both sides, it follows that

$$
\begin{aligned}
\|\widehat{h}\| & =\inf \left\{\|f \oplus g+h \oplus(-h)\|: f \oplus g \in \mathcal{H}, \quad h \oplus(-h) \in \mathcal{M}^{\perp}\right\}=\left\|P_{\mathcal{M}} \widetilde{k}\right\| \\
& =\left\|P_{\mathcal{M}}(f \oplus g)\right\|=\|f+g\| .
\end{aligned}
$$


Taking the square, we get

$$
\|f+g\|^{2}=\inf \left\{\|f \oplus g+h \oplus(-h)\|^{2}: f \oplus g \in \mathcal{H}, \quad h \oplus(-h) \in \mathcal{M}^{\perp}\right\}
$$

Then,

$$
\begin{aligned}
\| & f \oplus g+h \oplus(-h) \|^{2}=\langle f \oplus g+h \oplus(-h), f \oplus g+h \oplus(-h)\rangle \\
& =\langle f \oplus g, f \oplus g\rangle+\langle f \oplus g, h \oplus(-h)\rangle+\langle h \oplus(-h), f \oplus g\rangle+\langle h \oplus(-h), h \oplus(-h)\rangle \\
& =\langle f, f\rangle+\langle g, g\rangle+\langle f, h\rangle+\langle g,-h\rangle+\langle h, f\rangle+\langle-h, g\rangle+\langle h, h\rangle+\langle-h,-h\rangle \\
& =\|f+h\|^{2}+\|g-h\|^{2} .
\end{aligned}
$$

Hence, we get

$$
\begin{gathered}
\|f+g\|^{2}=\min \left\{\|f+h\|^{2}+\|g-h\|^{2}: f \in \mathcal{H}_{K^{(1)}}(X), g \in \mathcal{H}_{K^{(2)}}(X),\right. \\
\left.h \in \mathcal{H}_{K^{(1)}}(X) \cap \mathcal{H}_{K^{(2)}}(X)\right\} .
\end{gathered}
$$

Given Hilbert spaces $\mathcal{H}_{K^{(1)}}(X)$ and $\mathcal{H}_{K^{(2)}}(X)$, the intersection $\mathcal{H}_{K^{(1)}}(X) \cap$ $\mathcal{H}_{K^{(2)}}(X)$ will be again a Hilbert space of functions on $X$ with respect to the norm

$$
\|f\|^{2}:=\|f\|_{K^{(1)}}^{2}+\|f\|_{K^{(2)}}^{2} .
$$

Since every point evaluation functional is continuous in both $\mathcal{H}_{K^{(1)}}(X)$ and $\mathcal{H}_{K^{(2)}}(X)$, letting $f \in \mathcal{H}_{K^{(1)}}(X) \cap \mathcal{H}_{K^{(2)}}(X)$, it follows that every point evaluation functional will be continuous in $\mathcal{H}_{K^{(1)}}(X) \cap \mathcal{H}_{K^{(2)}}(X)$. Therefore the intersection Hilbert space is a RKHS.

Theorem 2.2.3. The reproducing kernel of the space

$$
\mathcal{H}_{K}(X)=\mathcal{H}_{K^{(1)}}(X) \cap \mathcal{H}_{K^{(2)}}(X)
$$

is determined, as a quadratic form, by

$\sum_{x, y} \overline{\varepsilon_{y}} \varepsilon_{x} K(y, x)=\inf \left\{\sum_{x, y} \overline{\eta_{y}} \eta_{x} K^{(1)}(y, x)+\sum_{x, y} \overline{\zeta_{y}} \zeta_{x} K^{(2)}(y, x):\left[\varepsilon_{x}\right]=\left[\eta_{x}\right]+\left[\zeta_{x}\right]\right\}$

where $\left[\epsilon_{x}\right]$ is an arbitrary complex valued function on $X$ with finite support, and the same are true for $\left[\eta_{x}\right]$ and $\left[\zeta_{x}\right]$. 
Proof. Consider the isometric map $S$, such that it embeds $\mathcal{H}_{K}(X)$ into the direct sum Hilbert space $\mathcal{H}_{K^{(1)}}(X) \oplus \mathcal{H}_{K^{(2)}}(X), S: \mathcal{H}_{K}(X) \longrightarrow \mathcal{H}_{K^{(1)}}(X) \oplus \mathcal{H}_{K^{(2)}}(X)$ that is

$$
S f=f \oplus f \text { for } f \in \mathcal{H}_{K}(X) .
$$

Let $P_{\mathcal{M}}$ be the orthogonal projection onto $\operatorname{Ran}(S):=\mathcal{M}$. Then $P_{\mathcal{M}}=S S^{*}$, and by using the reproducing and algebraic direct sum properties, it follows

$$
\begin{aligned}
(S f)(x) & =\left\langle S f, K_{x}^{(1)} \oplus K_{x}^{(2)}\right\rangle=\left\langle f \oplus f, K_{x}^{(1)} \oplus K_{x}^{(2)}\right\rangle \\
& =\left\langle f, K_{x}^{(1)}\right\rangle_{K^{(1)}}+\left\langle f, K_{x}^{(2)}\right\rangle_{K^{(2)}} \\
& =f(x)+f(x)=2 f(x) \text { where } f \in \mathcal{H}_{K}(X) .
\end{aligned}
$$

So, $\left\langle S f, K_{x}^{(1)} \oplus K_{x}^{(2)}\right\rangle=2 f(x)$, i.e. $\frac{1}{2}\left\langle S f, K_{x}^{(1)} \oplus K_{x}^{(2)}\right\rangle=f(x)$. This implies

$$
\frac{1}{2}\left\langle f, S^{*}\left(K_{x}^{(1)} \oplus K_{x}^{(2)}\right)\right\rangle=f(x)
$$

or equivalently

$$
\left\langle f, \frac{1}{2} S^{*}\left(K_{x}^{(1)} \oplus K_{x}^{(2)}\right)\right\rangle=f(x) .
$$

In other words, $K_{x}=\frac{1}{2} S^{*}\left(K_{x}^{(1)} \oplus K_{x}^{(2)}\right)$ for $x \in X$. Then using this and the isometricity of $\mathrm{S}$,

$$
\begin{aligned}
\sum_{x, y} \overline{\varepsilon_{y}} \varepsilon_{x} K(y, x) & =\left\|\sum_{x} \varepsilon_{x} K_{x}\right\|_{K}^{2}=\left\|\sum_{x} \varepsilon_{x} \frac{1}{2} S^{*}\left(K_{x}^{(1)} \oplus K_{x}^{(2)}\right)\right\|^{2} \\
& =\left\|\frac{1}{2} S S^{*} \sum_{x} \varepsilon_{x}\left(K_{x}^{(1)} \oplus K_{x}^{(2)}\right)\right\|^{2} \\
& =\left\|P_{\mathcal{M}}\left(\sum_{x} \varepsilon_{x}\left(K_{x}^{(1)} \oplus K_{x}^{(2)}\right)\right)\right\|^{2} .
\end{aligned}
$$

Now, since $\mathcal{M}=\operatorname{Ran}(S)=(\operatorname{ker}(S))^{\perp}$, then $\mathcal{M}=\left(\left\{K_{x}^{(1)} \oplus\left(-K_{x}^{(2)}\right): x \in X\right\}\right)^{\perp}$ which implies

$$
\begin{aligned}
\mathcal{M}^{\perp} & =\underline{\left[\left\{K_{x}^{(1)} \oplus\left(-K_{x}^{(2)}\right): x \in X\right\}\right]^{\perp \perp}} \\
& =\overline{\operatorname{lin}\left\{K_{x}^{(1)} \oplus\left(-K_{x}^{(2)}\right): x \in X\right\}} \\
& =\left\{K_{x}^{(1)} \oplus\left(-K_{x}^{(2)}\right): x \in X\right\} .
\end{aligned}
$$


So, the elements of the form $\frac{1}{2} \sum_{x} \lambda_{x}\left(K_{x}^{(1)} \oplus\left(-K_{x}^{(2)}\right)\right)$ are dense in $\mathcal{M}^{\perp}$. Then, by using Theorem 2.1 .12 and the property of orthogonal projection, we get

$$
\begin{aligned}
\| P_{\mathcal{M}}( & \left.\frac{1}{2} \sum_{x} \varepsilon_{x}\left(K_{x}^{(1)} \oplus K_{x}^{(2)}\right)\right) \|^{2} \\
= & \left\|\frac{1}{2} \sum_{x} \varepsilon_{x}\left(K_{x}^{(1)} \oplus K_{x}^{(2)}\right) \oplus \frac{1}{2} \sum_{x} \varepsilon_{x}\left(K_{x}^{(1)} \oplus K_{x}^{(2)}\right)\right\|^{2} \\
= & \left\langle\frac{1}{2} \sum_{x} \varepsilon_{x}\left(K_{x}^{(1)} \oplus K_{x}^{(2)}\right) \oplus \frac{1}{2} \sum_{x} \varepsilon_{x}\left(K_{x}^{(1)} \oplus K_{x}^{(2)}\right),\right. \\
& \left.\frac{1}{2} \sum_{x} \varepsilon_{x}\left(K_{x}^{(1)} \oplus K_{x}^{(2)}\right) \oplus \frac{1}{2} \sum_{x} \varepsilon_{x}\left(K_{x}^{(1)} \oplus K_{x}^{(2)}\right)\right\rangle \\
= & \left\langle\frac{1}{2} \sum_{x} \varepsilon_{x}\left(K_{x}^{(1)} \oplus K_{x}^{(2)}\right), \frac{1}{2} \sum_{x} \varepsilon_{x}\left(K_{x}^{(1)} \oplus K_{x}^{(2)}\right)\right\rangle \\
& +\left\langle\frac{1}{2} \sum_{x} \varepsilon_{x}\left(K_{x}^{(1)} \oplus K_{x}^{(2)}\right), \frac{1}{2} \sum_{x} \varepsilon_{x}\left(K_{x}^{(1)} \oplus K_{x}^{(2)}\right)\right\rangle \\
= & \left\|\frac{1}{2} \sum_{x} \varepsilon_{x}\left(K_{x}^{(1)} \oplus K_{x}^{(2)}\right)\right\|^{2}+\left\|\frac{1}{2} \sum_{x} \varepsilon_{x}\left(K_{x}^{(1)} \oplus K_{x}^{(2)}\right)\right\|^{2} \\
= & \frac{1}{2}\left\|\sum_{x} \varepsilon_{x}\left(K_{x}^{(1)} \oplus K_{x}^{(2)}\right)\right\|^{2} .
\end{aligned}
$$

Let $\frac{1}{2}\left(\varepsilon_{x}+\lambda_{x}\right)=\eta_{x}, \frac{1}{2}\left(\varepsilon_{x}-\lambda_{x}\right)=\delta_{x}$ and $\frac{1}{2}\left(\varepsilon_{x}+\lambda_{x}\right)+\frac{1}{2}\left(\varepsilon_{x}-\lambda_{x}\right)=\varepsilon_{x}$, that is,

$$
\left[\varepsilon_{x}\right]=\left[\eta_{x}\right]+\left[\delta_{x}\right]
$$

Finally, applying Theorem 2.2 .2 to the function $\frac{1}{2} \sum_{x} \varepsilon_{x}\left(K_{x}^{(1)} \oplus K_{x}^{(2)}\right)$, we get

$$
\frac{1}{2}\left\|\sum_{x} \varepsilon_{x}\left(K_{x}^{(1)} \oplus K_{x}^{(2)}\right)\right\|^{2}=\inf _{\left[\lambda_{x}\right]}\left\|\left(\sum_{x} \frac{1}{2}\left(\varepsilon_{x}+\lambda_{x}\right) K_{x}\right) \oplus\left(\sum_{x} \frac{1}{2}\left(\varepsilon_{x}-\lambda_{x}\right) K_{x}\right)\right\|^{2}
$$

where

$$
\begin{aligned}
\|\left(\sum_{x}\right. & \left.\frac{1}{2}\left(\varepsilon_{x}+\lambda_{x}\right) K_{x}\right) \oplus\left(\sum_{x} \frac{1}{2}\left(\varepsilon_{x}-\lambda_{x}\right) K_{x}\right) \|^{2} \\
& =\left\|\left(\sum_{x} \eta_{x} K_{x}^{(1)}\right) \oplus\left(\sum_{x} \delta_{x} K_{x}^{(2)}\right)\right\|^{2} \\
& =\left\langle\sum_{x} \eta_{x} K_{x}^{(1)} \oplus \sum_{x} \delta_{x} K_{x}^{(2)}, \sum_{x} \eta_{x} K_{x}^{(1)} \oplus \sum_{x} \delta_{x} K_{x}^{(2)}\right\rangle \\
& =\left\|\sum_{x} \eta_{x} K_{x}^{(1)}\right\|_{K^{(1)}}^{2}+\left\|\sum_{x} \delta_{x} K_{x}^{(2)}\right\|_{K^{(2)}}^{2} \\
& =\sum_{x, y} \overline{\eta_{y}} \eta_{x} K^{(1)}(y, x)+\sum_{x, y} \overline{\delta_{y}} \delta_{x} K^{(2)}(y, x)
\end{aligned}
$$

which completes the proof. 
Remark 2.2.4. Consider the tensor product Hilbert space $\mathcal{H}_{K^{(1)}}(X) \otimes \mathcal{H}_{K^{(2)}}(X)$. Take $g \in \mathcal{H}_{K^{(1)}}(X), h \in \mathcal{H}_{K^{(2)}}(X)$ and $x, x^{\prime} \in X$. It follows

$$
(g \otimes h)\left(x, x^{\prime}\right)=g(x) h\left(x^{\prime}\right)=\left\langle g, K_{x}^{(1)}\right\rangle\left\langle h, K_{x^{\prime}}^{(2)}\right\rangle=\left\langle g \otimes h, K_{x}^{(1)} \otimes K_{x^{\prime}}^{(2)}\right\rangle
$$

which shows that the tensor product Hilbert space $\mathcal{H}_{K^{(1)}}(X) \otimes \mathcal{H}_{K^{(2)}}(X)$ is a RKHS on $X \times X$.

Consider the map $\phi: X \longrightarrow \mathcal{H}_{K^{(1)}}(X) \otimes \mathcal{H}_{K^{(2)}}(X)$ defined by $x \mapsto K_{x}^{(1)} \otimes K_{x}^{(2)}$. Then

$$
\begin{aligned}
K(y, x) & =\left\langle\phi_{x}, \phi_{y}\right\rangle=\left\langle K_{x}^{(1)} \otimes K_{x}^{(2)}, K_{y}^{(1)} \otimes K_{y}^{(2)}\right\rangle \\
& =\left\langle K_{x}^{(1)}, K_{y}^{(1)}\right\rangle \cdot\left\langle K_{x}^{(2)}, K_{y}^{(2)}\right\rangle \\
& =K^{(1)}(y, x) \cdot K^{(2)}(y, x) \text { for } x, y \in X .
\end{aligned}
$$

Hence the pointwise product of two positive definite kernels is again a positive definite kernel.

Theorem 2.2.5. For the product kernel $K(y, x)=K^{(1)}(y, x) \cdot K^{(2)}(y, x)$, the RKHS $\mathcal{H}_{K}(X)$ consists of all functions $f$ on $X$ for which there are sequences $\left(g_{n}\right)_{n \geq 0}$ of functions in $\mathcal{H}_{K^{(1)}}(X)$ and $\left(h_{n}\right)_{n \geq 0}$ of functions in $\mathcal{H}_{K^{(2)}}(X)$ such that

$$
\sum_{1}^{\infty}\left\|g_{n}\right\|_{K^{(1)}}^{2}\left\|h_{n}\right\|_{K^{(2)}}^{2}<\infty \text { and } \sum_{1}^{\infty} g_{n}(x) h_{n}(x)=f(x) \text { for all } x \in X,
$$

and the norm is given by

$$
\|f\|_{K}^{2}=\min \left\{\sum_{1}^{\infty}\left\|g_{n}\right\|_{K^{(1)}}^{2}\left\|h_{n}\right\|_{K^{(2)}}^{2}\right\},
$$

where the minimum is taken over the set of all sequences $\left(g_{n}\right)_{n \geq 0}$ and $\left(h_{n}\right)_{\geq 0}$ satisfying (2.14).

Proof. Let $T$ be an operator from $\mathcal{H}_{K^{(1)}} \otimes \mathcal{H}_{K^{(2)}}$ to the space of functions on $X$, associated with $\phi_{x}$, as in Theorem 2.1 .12 more precisely,

$$
T: \mathcal{H}_{K^{(1)}} \otimes \mathcal{H}_{K^{(2)}} \longrightarrow \mathcal{F}(\mathcal{X}):=\{f: X \longrightarrow \mathbb{C}: f \text { complex function on } X\}
$$


Let $F \in \mathcal{H}_{K^{(1)}} \otimes \mathcal{H}_{K^{(2)}}$. Then $F$ will be of the form

$$
F=\sum_{1}^{\infty} g_{n} \otimes h_{n} \text { with } g_{n} \in \mathcal{H}_{K^{(1)}} \text { and } h_{n} \in \mathcal{H}_{K^{(2)}} .
$$

Let $x \in X$ and $\phi_{x}:=K_{x}^{(1)} \otimes K_{x}^{(2)}, \quad \phi_{x}: X \longrightarrow \mathcal{H}_{K^{(1)}} \otimes \mathcal{H}_{K^{(2)}}$ and $K(y, x)=$ $\left\langle\phi_{x}, \phi_{y}\right\rangle$.

It follows by Theorem 2.1 .12

$$
\begin{aligned}
(T F)(x) & =\left\langle F, \phi_{x}\right\rangle=\left\langle F, K_{x}^{(1)} \otimes K_{x}^{(2)}\right\rangle=\left\langle\sum_{n=1}^{\infty} g_{n} \otimes h_{n}, K_{x}^{(1)} \otimes K_{x}^{(2)}\right\rangle \\
& =\sum_{n=1}^{\infty}\left\langle g_{n}, K_{x}^{(1)}\right\rangle\left\langle h_{n}, K_{x}^{(2)}\right\rangle \\
& =\sum_{n=1}^{\infty} g_{n}(x) h_{n}(x)=f(x) .
\end{aligned}
$$

Finally,

$$
\begin{aligned}
\|F\|^{2} & =\langle F, F\rangle=\left\langle\sum_{n=1}^{\infty} g_{n} \otimes h_{n}, \sum_{n=1}^{\infty} g_{n} \otimes h_{n}\right\rangle \\
& =\sum_{n=1}^{\infty}\left\langle g_{n}, g_{n}\right\rangle_{K^{(1)}}\left\langle h_{n}, h_{n}\right\rangle_{K^{(2)}} \\
& =\sum_{n=1}^{\infty}\left\|g_{n}\right\|_{K^{(1)}}^{2}\left\|h_{n}\right\|_{K^{(2)}}^{2} .
\end{aligned}
$$

Taking the norm of $(T F)(x)=f(x)$, again by Theorem 2.1.12 we get

$$
\|f(x)\|_{K}=\|T F\|_{K}=\left\|P_{\mathcal{M}} F\right\|_{K}=\min \left\{\sum_{1}^{\infty}\left\|g_{n}\right\|_{K^{(1)}}^{2}\left\|h_{n}\right\|_{K^{(2)}}^{2}\right\} .
$$

Remark 2.2.6. If $X$ consists of a finite number of points, say $n$, then the space of all functions on $X$, that is $\mathbb{C}^{n}$, has the canonical RKHS structure $\left(l_{n}^{2},\langle\cdot, \cdot\rangle\right)$, where the point evaluation at $i$ is induced by the inner product with $e_{i},(i=1,2, \ldots, n)$. Moreover, for a positive definite kernel $K(j, i)$ on $X$, we have

$$
K(j, i)=\left\langle L e_{i}, e_{j}\right\rangle, \quad(i, j=1,2, \ldots, n)
$$

where $\mathrm{L}$ is a uniquely determined linear operator on $l_{n}^{2}$ and is positive definite. 
Theorem 2.2.7. If $K(j, i)$ is a strictly positive definite kernel on $X=$ $\{1,2, \ldots, n\}$ and the operator $L$ on $l_{n}^{2}$ is defined as in (2.15), then $L$ is a strictly positive definite operator and

$$
\langle f, g\rangle_{K}=\left\langle L^{-1} f, g\right\rangle \text { for } f, g \in \mathbb{C}^{n}
$$

Proof. Given $K(j, i)$ a positive definite kernel on $X=\{1,2, \ldots, n\}$, consider the inclusion map $J: \mathcal{H}_{K}(X) \longrightarrow \mathbb{C}^{n}=l_{n}^{2}$. As a result of Theorem 2.1.11, $J$ is continuous.

Let $J^{*}$ be the adjoint of $J$. We have $J^{*}: l_{n}^{2} \longrightarrow \mathcal{H}_{K}(X)$. Let $J^{*}\left(e_{i}\right)=K_{i}$, $(i=1,2, \ldots, n)$. By $(2.15)$,

$$
\left\langle L e_{i}, e_{j}\right\rangle=K(j, i)=\left\langle K_{i}, K_{j}\right\rangle_{K}=\left\langle J^{*} e_{i}, J^{*} e_{j}\right\rangle_{K}=\left\langle\left(J J^{*} e_{i}\right), e_{j}\right\rangle
$$

which gives

$$
L=J J^{*}
$$

Since $K$ is a strictly positive definite kernel, it follows that $\operatorname{dim}\left(\mathcal{H}_{K}(X)\right)=n$ and $J$ is a bijection. Hence,

$$
\begin{aligned}
\langle f, g\rangle_{K} & =\left\langle J^{-1} f, J^{-1} g\right\rangle_{K}=\left\langle\left(J^{-1}\right)^{*} J^{-1} f, g\right\rangle \\
& =\left\langle\left(J J^{*}\right)^{-1} f, g\right\rangle=\left\langle L^{-1} f, g\right\rangle
\end{aligned}
$$

and consequently we have

$$
\langle f, g\rangle_{K}=\left\langle L^{-1} f, g\right\rangle
$$

Each positive definite operator $L$ on $l_{n}^{2}$ produces a positive definite kernel $K(j, i)$ on $X$ by (2.15).

Theorem 2.2.8. If $L_{i},(i=1,2)$ are two strictly positive definite operators on $l_{n}^{2}$, then

$$
\left\langle\left(L_{1}^{-1}+L_{2}^{-1}\right) f, f\right\rangle=\min \left\{\left\langle L_{1} g, g\right\rangle+\left\langle L_{2} h, h\right\rangle: g+h=f\right\} \text { for } f \in \mathbb{C}^{n} .
$$


Proof. Let $K^{(1)}$ and $K^{(2)}$ be the kernels associated to $L_{1}$ and $L_{2}$, respectively. By using (2.15) and the result of previous theorem, we have

$$
\langle f, g\rangle_{K^{(i)}}=\left\langle L_{i}^{-1} f, g\right\rangle \text { for } f, g \in \mathbb{C}^{n} .
$$

Now consider the inner product $\langle f, f\rangle_{K^{(1)}+K^{(2)}}$ in Theorem 2.2.2,

$$
\begin{aligned}
\langle f, f\rangle_{K^{(1)}+K^{(2)}} & =\|f\|_{K^{(1)}+K^{(2)}}^{2}=\min \left\{\|g\|_{K^{(1)}}^{2}+\|h\|_{K(2)}^{2}: g+h=f\right\} \\
& =\min \left\{\langle g, g\rangle_{K^{(1)}}+\langle h, h\rangle_{K^{(2)}}: g+h=f\right\} \\
& =\min \left\{\left\langle L_{1} g, g\right\rangle+\left\langle L_{2} h, h\right\rangle: g+h=f\right\} \text { for } f, g \text { and } h \in \mathbb{C}^{n},
\end{aligned}
$$

and since by $(2.16)$ in Theorem 2.2.7, we have $\langle f, f\rangle=\left\langle\left(L_{1}^{-1}+L_{2}^{-1}\right)^{-1} f, f\right\rangle$, combining this with the above equations, we obtain

$$
\begin{aligned}
\langle f, f\rangle & =\left\langle\left(L_{1}^{-1}+L_{2}^{-1}\right)^{-1} f, f\right\rangle \\
& =\min \left\{\left\langle L_{1} g, g\right\rangle+\left\langle L_{2} h, h\right\rangle: g+h=f \text { for } f \in \mathbb{C}^{n}\right\} .
\end{aligned}
$$

\subsection{Extension of Functions and Kernels}

The following four theorems refer to extensions of a function (respectively a kernel), defined on a subset, to a function (respectively a kernel) on the whole set which obeys suitable restrictions.

Theorem 2.3.1. Let $K(y, x)$ be a positive definite kernel on $X$ and $h$ a function on $X_{0}$, where $X_{0}$ is a subset of $X$. If

$$
[h(y) \overline{h(x)}] \leq[K(y, x)] \text { on } X_{0}
$$

then there is a function $\widetilde{h} \in \mathcal{H}_{K}(X)$ such that

$$
\|\widetilde{h}\|_{K} \leq 1 \text { and } \tilde{h}(x)=h(x) \text { for } x \in X_{0} .
$$

Proof. Let $K^{(0)}(y, x)$ be the restriction of $K(y, x)$ to $X_{0}$. We know that $K^{(0)}(y, x)$ is positive definite because $K(y, x)$ is positive definite. By assumption, for $h$ a function on $X_{0}$, since the equation (2.18) is satisfied, then applying Theorem 
2.1.10 with $\gamma=1$, we have $h \in \mathcal{H}_{K^{(0)}}\left(X_{0}\right)$ and by the proof of Theorem 2.1.10, $\|h\|_{K^{(0)}} \leq \gamma=1$. It follows by Theorem 2.2.1 that, for $\mathcal{H}_{K^{(0)}}\left(X_{0}\right)$ and $\mathcal{H}_{K}(X)$ are reproducing kernel Hilbert spaces we have

$$
\mathcal{H}_{K^{(0)}}\left(X_{0}\right)=\left\{\left.\widetilde{h}\right|_{X_{0}}: \widetilde{h} \in \mathcal{H}_{K}(X)\right\}
$$

and

$$
\|h\|_{K^{(0)}}=\min \left\{\|\widetilde{h}\|_{K}:\left.\widetilde{h}\right|_{X_{0}}=h\right\} \text { for } h \in \mathcal{H}_{K^{(0)}}\left(X_{0}\right),
$$

equivalently,

$\|h\|_{K^{(0)}}=\min \left\{\|\widetilde{h}\|_{K}: \widetilde{h}(x)=h(x), x \in X_{0}\right\}$ for $h \in \mathcal{H}_{K^{(0)}}\left(X_{0}\right)$ with $\|h\|_{K^{(0)}} \leq 1$.

Hence,

$$
\|\widetilde{h}\|_{K} \leq 1
$$

Theorem 2.3.2. Let $K^{(i)}(y, x),(i=1,2)$ be positive definite kernels on $X, h$ a function on $X_{0} \subseteq X$.

(i) If

$$
[h(y) \overline{h(x)}] \leq\left[K^{(1)}(y, x)+K^{(2)}(y, x)\right] \text { on } X_{0},
$$

then there are functions $\tilde{f} \in \mathcal{H}_{K^{(1)}}(X)$ and $\widetilde{g} \in \mathcal{H}_{K^{(2)}}(X)$ such that

$$
\|\widetilde{f}\|_{K^{(1)}}^{2}+\|\widetilde{g}\|_{K^{(2)}}^{2} \leq 1 \text { and } h(x)=\widetilde{f}(x)+\widetilde{g}(x) \text { for } x \in X_{0} .
$$

(ii) If

$$
[h(y) \overline{h(x)}] \leq\left[K^{(1)}(y, x) \cdot K^{(2)}(y, x)\right] \text { on } X_{0},
$$

then there are sequences of functions $\left(f_{n}\right)_{n \geq 1} \subset \mathcal{H}_{K^{(1)}}(X)$ and $\left(g_{n}\right)_{n \geq 1} \subset$ $\mathcal{H}_{K^{(2)}}(X)$ such that

$$
\sum_{1}^{\infty}\left\|f_{n}\right\|_{K^{(1)}}^{2}\left\|g_{n}\right\|_{K^{(2)}}^{2} \leq 1 \text { and } \sum_{1}^{\infty} f_{n}(x) g_{n}(x)=h(x) \text { for } x \in X_{0} .
$$

Proof. (i) For this part, consider the kernel $K(y, x)=K^{(1)}(y, x)+K^{(2)}(y, x)$ on $X$. We know that $K(y, x)$ is positive definite because $K^{(1)}$ and $K^{(2)}$ are positive definite . 
Let $\mathcal{H}_{K^{(1)}}(X)$ and $\mathcal{H}_{K^{(2)}}(X)$ be associated reproducing kernel Hilbert spaces for $K^{(1)}$ and respectively $K^{(2)}$ their reproducing kernels. By Theorem 2.2.2, we have $\mathcal{H}_{K}$, the reproducing kernel Hilbert space admitting the reproducing kernel $K(y, x)$ such that $\mathcal{H}_{K}(X)=\mathcal{H}_{K^{(1)}}(X)+\mathcal{H}_{K^{(2)}}(X)$. By assumption, since

$$
[h(y) \overline{h(x)}] \leq\left[K^{(1)}(y, x)+K^{(2)}(y, x)\right]=K(y, x) \text { on } X_{0},
$$

applying previous theorem, there exists $\widetilde{h} \in \mathcal{H}_{K}(x)$ such that $\|\widetilde{h}\|_{K} \leq 1$ and $\widetilde{h}(x)=h(x)$ for $x \in X_{0}$.

Returning to Theorem 2.2.2, there are functions $\tilde{f} \in \mathcal{H}_{K^{(1)}}(X)$ and $\widetilde{g} \in$ $\mathcal{H}_{K^{(2)}}(X)$ such that $\widetilde{h}=\widetilde{f}+\widetilde{g}$ and

$$
\|\widetilde{h}\|_{K}^{2}=\min \left\{\|\widetilde{f}+k\|_{K^{(1)}}^{2}+\|\widetilde{g}-k\|_{K^{(2)}}^{2}: k \in \mathcal{H}_{K^{(1)}} \cap \mathcal{H}_{K^{(2)}}\right\} \leq 1 .
$$

Hence, we get

$$
\|\widetilde{h}\|_{K}^{2}=\|\widetilde{f}\|_{K^{(1)}}^{2}+\|\widetilde{g}\|_{K^{(2)}}^{2} \leq 1 \quad \text { and } \quad h(x)=\widetilde{f}(x)+\widetilde{g}(x) \text { for } x \in X_{0} .
$$

(ii) Consider $K(y, x)$, the pointwise product of two kernels $K^{(1)}(y, x)$ and $K^{(2)}(y, x)$, that is $K(y, x)=K^{(1)}(y, x) \cdot K^{(2)}(y, x)$. Since both $K^{(1)}$ and $K^{(2)}$ are positive definite, then $K(y, x)$ is also positive definite, see Remark 2.2.4. Similarly as in (i), let $\mathcal{H}_{K^{(1)}}(X)$ and $\mathcal{H}_{K^{(2)}}(X)$ be the associated reproducing kernel Hilbert spaces for $K^{(1)}$ and $K^{(2)}$ their reproducing kernels, respectively. Denote by $\mathcal{H}_{K}(X)$ the reproducing kernel Hilbert space admitting the reproducing kernel $K(y, x)$.

Since, by assumption,

$$
[h(y) \overline{h(x)}] \leq\left[K^{(1)}(y, x) \cdot K^{(2)}(y, x)\right]=K(y, x) \text { on } X_{0},
$$

then there exists $\widetilde{h} \in \mathcal{H}_{K}(X)$ such that $\|\widetilde{h}\|_{K} \leq 1$ and $\widetilde{h}(x)=h(x)$ for $x \in X_{0}$.

By Theorem 2.2.5, the reproducing kernel Hilbert space $\mathcal{H}_{K}$ consists of all functions $\widetilde{h}$ on $X$ such that there exist sequences $\left(f_{n}\right)_{n \geq 1} \subset \mathcal{H}_{K^{(1)}}(X)$ and $\left(g_{n}\right)_{n \geq 1} \subset \mathcal{H}_{K^{(2)}}(X)$ subject to the conditions:

$$
\widetilde{h}(x)=\sum_{1}^{\infty} f_{n}(x) g_{n}(x), \quad \text { for } x \in X_{0}
$$


and

$$
\sum_{1}^{\infty}\left\|f_{n}\right\|_{K^{(1)}}^{2}\left\|g_{n}\right\|_{K^{(2)}}^{2}<\infty
$$

with

$$
\|\widetilde{h}\|_{K}^{2}=\min \left\{\sum_{1}^{\infty}\left\|f_{n}\right\|_{K^{(1)}}^{2}\left\|g_{n}\right\|_{K^{(2)}}^{2}\right\} .
$$

But we know $\|\widetilde{h}\|_{K}^{2} \leq 1$. Hence we have

$$
\sum_{1}^{\infty}\left\|f_{n}\right\|_{K^{(1)}}^{2}\left\|g_{n}\right\|_{K^{(2)}}^{2} \leq 1
$$

As a result of the above theorem and Theorem 2.1.13, any positive definite kernel $K(y, x)$ on a subset $X_{0}$ of $X$ can be extended to a positive definite kernel on the whole set $X$.

Let $K(y, x)$ be a positive definite kernel on $X$. For a continuous linear operator $L$ on $\mathcal{H}_{K}(X)$, (i.e. $L: \mathcal{H}_{K}(X) \longrightarrow \mathcal{H}_{K}(X)$ ), we associate its kernel as

$$
T(y, x)=\left\langle L K_{x}, K_{y}\right\rangle \text { for } x, y \in X
$$

Recall that $\left\{K_{x}: x \in X\right\}$ is total in $\mathcal{H}_{K}(X)$. For any finitely supported $\left[\xi_{x}\right]$ and $\left[\eta_{x}\right]$, we have

$$
\left\langle L\left(\sum_{x} \xi_{x} K_{x}\right), \sum_{y} \eta_{y} K_{y}\right\rangle=\sum_{x, y} \xi_{x} \bar{\eta}_{y}\left\langle L K_{x}, K_{y}\right\rangle=\sum_{x, y} \xi_{x} \bar{\eta}_{y} T(y, x) .
$$

Therefore, $\|L\| \leq 1$ is equivalent to the following condition:

$$
\begin{aligned}
\left|\sum_{x, y} \xi_{x} \bar{\eta}_{y} T(y, x)\right|^{2} & =\left|\left\langle L\left(\sum_{x} \xi_{x} K_{x}\right), \sum_{y} \eta_{y} K_{y}\right\rangle\right|^{2} \\
& \leq\left\|\sum_{x} \xi_{x} K_{x}\right\|^{2}\left\|\sum_{y} \eta_{y} K_{y}\right\|^{2} \\
& =\left(\sum_{x, y} \xi_{x} \bar{\xi}_{y} K(y, x)\right)\left(\sum_{x, y} \eta_{x} \bar{\eta}_{y} K(y, x)\right) .
\end{aligned}
$$

Thus,

$$
\left|\sum_{x, y} \xi_{x} \bar{\eta}_{y} T(y, x)\right|^{2} \leq\left(\sum_{x, y} \xi_{x} \bar{\xi}_{y} K(y, x)\right)\left(\sum_{x, y} \eta_{x} \bar{\eta}_{y} K(y, x)\right)
$$


This last condition (2.23) can be expressed as the positive definiteness of the kernel $\widetilde{K}$ on the disjoint union of $X$ and its copy $X^{\prime}$, where $\widetilde{K}$ is defined as

$$
\widetilde{K}(y, x)= \begin{cases}K(y, x) & \text { if } x, y \in X \\ T\left(y, x^{\prime}\right) & \text { if } x \in X^{\prime}, y \in X \\ \overline{T\left(x, y^{\prime}\right)} & \text { if } x \in X, y \in X^{\prime} \\ K\left(y^{\prime}, x^{\prime}\right) & \text { if } x, y \in X^{\prime}\end{cases}
$$

where the canonical identification map $X^{\prime} \longrightarrow X$ is denoted by prime and the positive definiteness will be denoted by

$$
\left[\begin{array}{ll}
{[K(y, x)]} & {[T(y, x)]} \\
{[\overline{T(x, y)}]} & {[K(y, x)]}
\end{array}\right] \geq 0 \text { on } X
$$

Conversely, if a kernel $T(y, x)$ is given on $X$ and satisfies one of the equations (2.23) or (2.24), then there is a continuous linear operator $L$ on $\mathcal{H}_{K}(X)$ with $\|L\| \leq 1$ whose kernel coincides with $T$.

Lemma 2.3.3. Let $L$ be a bounded linear operator on $\mathcal{H}_{K}$ and $T$ the associated kernel as in (2.22). Then $L$ is self-adjoint (respectively positive) if and only if the kernel $T(y, x)$ is Hermitian (respectively positive definite).

Proof. $L$ is self-adjoint if and only if $L=L^{*}$, equivalently for all $x, y \in X$,

$$
T(y, x)=\left\langle L K_{x}, K_{y}\right\rangle=\left\langle L^{*} K_{x}, K_{y}\right\rangle=\overline{\left\langle K_{y}, L^{*} K_{x}\right\rangle}=\overline{T(x, y)} .
$$

Moreover, $L$ is positive if and only if

$$
\langle L f, f\rangle \geq 0, \text { for all } f \in \mathcal{H}_{K},
$$

equivalently, for any finitely supported family of complex numbers $\left\{\epsilon_{x}\right\}_{x \in X}$,

$$
\sum_{x, y} \bar{\epsilon}_{y} \xi_{x} L(y, x) \geq 0
$$

Theorem 2.3.4. If a kernel $T(y, x)$ on $X_{0}$ satisfies

$$
\left[\begin{array}{ll}
{[K(y, x)]} & {[T(y, x)]} \\
{[\overline{T(y, x)}]} & {[K(y, x)]}
\end{array}\right] \geq 0 \text { on } X_{0},
$$


then there exists a kernel $\tilde{T}(y, x)$ on $X$ such that $T(y, x)=\tilde{T}(y, x)$ for $x, y \in X_{0}$ and

$$
\left[\begin{array}{ll}
{[K(y, x)]} & {[T(y, x)]} \\
{[\overline{T(y, x)}]} & {[K(y, x)]}
\end{array}\right] \text { on } X \text {. }
$$

If $T(y, x)$ is Hermitian (respectively positive definite), then $\tilde{T}(y, x)$ can be chosen Hermitian (respectively positive definite).

Proof. Let $K^{(0)}(y, x)$ be the restriction of $K(y, x)$ to $X_{0}$. Consider the reproducing kernel Hilbert space $\mathcal{H}_{K^{(0)}}\left(X_{0}\right)$. Let $T(y, x)$ be a kernel on $X_{0}$ satisfying

$$
\left[\begin{array}{ll}
{[K(y, x)]} & {[T(y, x)]} \\
{[\overline{T(y, x)}]} & {[K(y, x)]}
\end{array}\right] \geq 0 \text { on } X_{0} .
$$

This implies that there is a continuous linear operator $L$ on $\mathcal{H}_{K^{(0)}}\left(X_{0}\right)$ such that $\|L\| \leq 1$ and $T(y, x)=\left\langle L K_{x}{ }^{(0)}, K_{y}{ }^{(0)}\right\rangle$ for $x, y \in X_{0}$. Then there is an isomorphism $S$ from $\mathcal{M}$, the closed linear span of $\left\{K_{x}: x \in X_{0}\right\}$, onto $\mathcal{H}_{K^{(0)}}\left(X_{0}\right)$ such that $S K_{x}=K_{x}^{(0)}$ for $x \in X_{0}$. Denote by $P_{\mathcal{M}}$ the orthogonal projection onto $\mathcal{M}$. Define

$$
\tilde{T}(y, x):=\left\langle L S P_{\mathcal{M}} K_{x}, S P_{\mathcal{M}} K_{y}\right\rangle \text { for } x, y \in X
$$

Then

$$
\tilde{T}(y, x):=\left\langle S^{*} L S P_{\mathcal{M}} K_{x}, P_{\mathcal{M}} K_{y}\right\rangle=\left\langle P_{\mathcal{M}} S^{*} L S P_{\mathcal{M}} K_{x}, K_{y}\right\rangle \text { for all } x, y \in X
$$

This means that $\tilde{T}(y, x)$ becomes the kernel of the continuous linear operator $P_{\mathcal{M}} S^{*} L S P_{\mathcal{M}}$ on $\mathcal{H}_{K}(X)$. If we take the norm of this operator, by using the properties of orthogonal projections and isometric operators, we get

$$
\begin{aligned}
\left\|P_{\mathcal{M}} S^{*} L S P_{\mathcal{M}}\right\| & \leq\left\|P_{\mathcal{M}}\right\|\left\|S^{*}\right\|\|L\|\|S\|\left\|P_{\mathcal{M}}\right\| \\
& \leq\left\|S^{*}\right\|\|L\|\|S\| \\
& \leq\|L\| \leq 1
\end{aligned}
$$

Then for any $x, y \in X_{0}$,

$$
\tilde{T}(y, x)=\left\langle L S P_{\mathcal{M}} K_{x}, S P_{\mathcal{M}} K_{y}\right\rangle=\left\langle L S K_{x}, S K_{y}\right\rangle
$$

and using $S K_{x}=K_{x}^{(0)}$ for $x \in X_{0}$, we get

$$
\left\langle L S K_{x}, S K_{y}\right\rangle=\left\langle L K_{x}{ }^{(0)}, K_{y}{ }^{(0)}\right\rangle=T(y, x)
$$


and hence

$$
T(y, x)=\tilde{T}(y, x) .
$$

Moreover, if $T(y, x)$ is Hermitian (respectively positive definite), then $L^{*}$ is self-adjoint (respectively positive definite) by previous remark and so the operator $P_{\mathcal{M}} S^{*} L S P_{\mathcal{M}}$ will be self-adjoint (respectively positive definite). Denote $P_{\mathcal{M}} S^{*} L S P_{\mathcal{M}}=N$. Then

$$
\begin{aligned}
\tilde{T}(y, x) & =\left\langle P_{\mathcal{M}} S^{*} L S P_{M} K_{x}, K_{y}\right\rangle=\left\langle N K_{x}, K_{y}\right\rangle \\
& =\left\langle N^{*} K_{x}, K_{y}\right\rangle=\left\langle K_{x}, N K_{y}\right\rangle=\overline{\left\langle N K_{y}, K_{x}\right\rangle}=\overline{\langle\tilde{T}(x, y)\rangle} .
\end{aligned}
$$

So $\tilde{T}(y, x)$ is Hermitian (respectively positive definite).

When $L$ is self-adjoint, then $\|L\| \leq 1$ if and only if

$$
|\langle L f, f\rangle| \leq\|L\|\|f\|_{K}^{2} \leq\|f\|_{K}^{2} \quad \text { for all } f \in \mathcal{H}_{K}(X) .
$$

This implies

$$
\left|\left\langle L\left(\sum_{x} \xi_{x} K_{x}\right), \sum_{y} \xi_{y} K_{y}\right\rangle\right| \leq \sum_{x, y} \bar{\xi}_{y} \xi_{x} K(y, x),
$$

which is equivalent to

$$
\left|\sum_{x, y} \bar{\xi}_{y} \xi_{x} M(y, x)\right| \leq \sum_{x, y} \bar{\xi}_{y} \xi_{x} K(y, x), \text { for all }\left[\xi_{x}\right] .
$$

When $L$ is not self adjoint, then either of the equations (2.25) or equivalently (2.26) only imply $L \leq 2$.

Theorem 2.3.5. Let $K(y, x)$ be a positive definite kernel on $X$, and let $L(y, x)$ be a kernel on $X_{0}$. If for any finitely supported family of complex numbers $\left\{\xi_{x}\right\}_{x \in X}$

$$
\left|\sum_{x, y \in X_{0}} \bar{\xi}_{y} \xi_{x} L(y, x)\right| \leq \sum_{x, y \in X_{0}} \bar{\xi}_{y} \xi_{x} K(y, x),
$$

then there is a kernel $\tilde{L}(y, x)$ on $X$ such that $L(y, x)=\tilde{L}(y, x)$ for all $x, y \in X_{0}$ and

$$
\left|\sum_{x, y} \bar{\xi}_{y} \xi_{x} \tilde{L}(y, x)\right| \leq \sum_{x, y} \bar{\xi}_{y} \xi_{x} K(y, x) \text { for all }\left[\xi_{x}\right]
$$

If $L(y, x)$ is Hermitian (respectively positive definite), $\tilde{L}(y, x)$ can be chosen Hermitian (respectively positive definite). 
Proof. Let equation (2.27) be satisfied for $K(y, x)$ a positive definite kernel on $X$ and let $L(y, x)$ be a kernel on $X_{0}$. So this is equivalent with Theorem 2.3.4. This implies that the linear operator $L$ on $\mathcal{H}_{K}^{(0)}\left(X_{0}\right)$ satisfies

$$
\left|\langle L h, h\rangle_{K^{(0)}}\right| \leq\|h\|_{K^{(0)}}^{2} \text { for } h \in \mathcal{H}_{K}^{(0)}\left(X_{0}\right) .
$$

Taking $S$ isometric and choosing $\tilde{L}(y, x)=\left\langle L S P_{M} K_{x}, S P_{M} K_{y}\right\rangle$, similarly as in the previous proof, we have

$$
\left|\left\langle L S P_{M} f, S P_{M} f\right\rangle_{K^{(0)}}\right| \leq\left\|S P_{M} f\right\|_{K^{(0)}}^{2} \leq\|f\|_{K}^{2} \text { for } f \in \mathcal{H}_{K}(X) .
$$

Hence, similarly as in the previous proof, we find that $L(y, x)=\tilde{L}(y, x)$ for $x, y \in X_{0}$. Therefore,

$$
\begin{aligned}
\left|\sum_{x, y} \bar{\xi}_{y} \xi_{x} \tilde{L}(y, x)\right| & =\left|\sum_{x, y} \bar{\xi}_{y} \xi_{x}\left\langle P_{M} S^{*} L S P_{M} K_{x}, K_{y}\right\rangle\right| \\
& =\left|\left\langle P_{M} S^{*} L S P_{M}\left(\sum_{x} \xi_{x} K_{x}\right), \sum_{y} \xi_{y} K_{y}\right\rangle\right| \\
& \leq\left\|P_{M} S^{*} L S P_{M}\right\|\left\|\sum_{x} \xi_{x} K_{x}\right\|^{2} \\
& \leq \sum_{x, y} \bar{\xi}_{y} \xi_{x}\left\langle K_{x}, K_{y}\right\rangle \\
& \leq \sum_{x, y} \bar{\xi}_{y} \xi_{x} K(y, x) .
\end{aligned}
$$




\section{Chapter 3}

\section{Spaces of Analytic Functions}

\subsection{Sesqui-analytic kernels}

Definition 3.1.1. A two variable function on a domain $\Omega$ in the complex plane, is sesqui-analytic if it is analytic in the first variable and anti-analytic in the second variable.

For example, this holds if the kernel $K(w, z)$ is analytic in the first variable and Hermitian, that is,

$$
K(w, z)=K_{z}(w)=\overline{K_{w}(z)}=\overline{K(z, w)} \quad \text { for all } \quad w, z \in \Omega .
$$

Definition 3.1.2. A function $f$ defined on some topological space $X$ with real or complex values is called locally bounded, if for any $x_{0}$ in $X$, there exists a neighborhood $A$ of $x_{0}$ such that $f(A)$ is a bounded set, that is, for some number $M>0,|f(x)| \leq M$ for all $x$ in $A$.

We have the kernel $K(w, z)$ is locally bounded in the sense that it is bounded on $A \times B$ for every pair $\{A, B\}$ of compact subsets of a domain $\Omega$.

Let us denote by $\Omega$ a connected domain of the complex plane. 
Theorem 3.1.3. The reproducing kernel Hilbert space $\mathcal{H}_{K}(\Omega)$ consists of analytic functions on $\Omega$ if and only if the positive definite kernel $K(w, z)$ on $\Omega$ is sesquianalytic and locally bounded.

Proof. Suppose that $\mathcal{H}_{K}(\Omega)$ consists of analytic functions on $\Omega$. Let $K(w, z)$ be the reproducing kernel of $\mathcal{H}_{K}(\Omega)$ and note that $K_{z} \in \mathcal{H}_{K}$ is analytic. By the definition of a reproducing kernel which is positive definite, we have

$$
K(w, z)=\overline{K(z, w)} \quad \text { for all } w, z \in \Omega .
$$

So $K$ is sesqui-analytic. To see the localy boundedness, consider any pair $\{A, B\}$ of compact subsets of $\Omega$. By assumption, every $f \in \mathcal{H}_{K}(\Omega)$ is analytic. Then

$$
f(z)=\left\langle f, K_{z}\right\rangle, \quad z \in \Omega,
$$

is analytic and hence continuous in $z$. Then the map $z \longmapsto K_{z}$ is weakly continuous. This implies $\left\{K_{z}: z \in A\right\}$ is weakly compact, thus weakly bounded. Then by the Theorem A.3.6, weakly boundedness implies strong boundedness. That is, $\sup _{z \in A}\left\|K_{z}\right\|=: \gamma_{A}<\infty$. Now by Schwarz Inequality,

$$
|K(w, z)|=\left|\left\langle K_{z}, K_{w}\right\rangle\right| \leq\left\|K_{z}\right\| \cdot\left\|K_{w}\right\| \leq \gamma_{A} \cdot \gamma_{B},
$$

for $w \in A, z \in B$. Hence $K$ is locally bounded.

Conversely, suppose that a positive definite kernel $K(w, z)$ on $\Omega$ is sesquianalytic and locally bounded. Recall that the set $\left\{K_{z}: z \in \Omega\right\}$ is total in $\mathcal{H}_{K}(\Omega)$. Then for each $f \in \mathcal{H}_{K}(\Omega), f$ is the strong limit of a sequence $\left(f_{n}\right)_{n \geq 1}$ in the linear span of $\left\{K_{z}: z \in \Omega\right\}$, that is $\left\|f_{n}-f\right\| \longrightarrow 0$, as $n \rightarrow \infty$. By assumption, since $K(w, z)$ is sesqui-analytic, then $K(w, z)=K_{z}(w)$ is analytic in $w$. Then by reproducing property, since

$$
f_{n}(w)=\left\langle f_{n}, K_{w}\right\rangle, \quad w \in \Omega,
$$

it follows that $f_{n}$ is analytic. Since $K(w, z)$ is locally bounded, we have $\sup _{z \in A}\left\|K_{z}\right\| \equiv \gamma_{A}<\infty$, where $A$ is any compact subset of $\Omega$. Then for $w \in A$,

$$
\begin{aligned}
\left|f_{n}(w)-f(w)\right| & =\left|\left\langle f_{n}, K_{w}\right\rangle-\left\langle f, K_{w}\right\rangle\right|=\left|\left\langle f_{n}-f, K_{w}\right\rangle\right| \\
& =\left\|f_{n}-f\right\|\left\|K_{w}\right\| \leq \gamma_{A}\left\|f_{n}-f\right\|
\end{aligned}
$$


and since $\left\|f_{n}-f\right\| \longrightarrow 0$, (as $\left.n \rightarrow \infty\right)$, we have $f_{n}$ converges to $f$ uniformly on each compact subset $A$ of $\Omega$. Hence, $\mathcal{H}_{K}(\Omega)$ consists of analytic functions on $\Omega$.

Definition 3.1.4. A subset $\Lambda$ of $\Omega$ is called determining subset if every analytic function on $\Omega$, equal to zero on $\Lambda$, vanishes identically on $\Omega$. In particular, if $\Lambda$ has a limit point in $\Omega$, it is a determining subset.

If two analytic functions are equal on a determining subset $\Lambda$, then they coincide on the whole set $\Omega$, i.e. $\left.f_{1}\right|_{\Lambda}=\left.f_{2}\right|_{\Lambda}$ implies $\left.f_{1}\right|_{\Omega}=\left.f_{2}\right|_{\Omega}$.

In particular, given two sesqui-analytic kernels $K^{(1)}(w, z)$ and $K^{(2)}(w, z)$ on $\Omega$, if $K^{(1)}(w, z)=K^{(2)}(w, z)$ for $w, z \in \Lambda$ and $\Lambda$ is determining subset, then $K^{(1)}(w, z)=K^{(2)}(w, z)$ on whole $\Omega$.

Theorem 3.1.5. Let $K(w, z)$ be a locally bounded, sesqui-analytic and positive definite kernel on $\Omega$ and $\Lambda$ a determining subset of $\Omega$.

(i) If a function $h$ on $\Lambda$ satisfies the condition

$$
[h(w) \overline{h(z)}] \leq[K(w, z)] \text { on } \Lambda,
$$

then there exists uniquely an analytic function $\tilde{h}$ on $\Omega$ such that

$$
\tilde{h}(z)=h(z) \text { for } z \in \Lambda \text { and }[\tilde{h}(w) \bar{h}(z)] \leq[K(w, z)] .
$$

(ii) If a positive definite kernel $L(w, z)$ satisfies

$$
[L(w, z)] \leq[K(w, z)] \text { on } \Lambda,
$$

then there exists uniquely a sesqui-analytic positive definite kernel $\tilde{L}(w, z)$ on $\Omega$ such that

$$
\tilde{L}(w, z)=L(w, z) \text { for } w, z \in \Lambda \text { and }[\tilde{L}(w, z)] \leq[K(w, z)] \text { on } \Omega \text {. }
$$

Proof. Let $K(w, z)$ be a locally bounded, sequi-analytic and positive definite kernel on $\Omega$ and $\Lambda$ a determining subset of $\Omega$. 
(i) Suppose that for a function $h$ on $\Omega(3.1)$ is satisfied. Then by Theorem 2.3.1, there exists $\tilde{h} \in \mathcal{H}_{K}(\Omega)$ such that $\|\tilde{h}\|_{K} \leq 1$ and $\tilde{h}(z)=h(z)$ for $z \in \Lambda$. By the same theorem, we can extend $h(w) \overline{h(z)}$ on $\Lambda$ to a positive definite kernel $\tilde{h}(w) \overline{\tilde{h}(z)}$ on $\Omega$. Then $\tilde{h}(w) \overline{\tilde{h}(z)}=h(w) \overline{h(z)}$ on $\Lambda$ implies that

$$
[\tilde{h}(w) \overline{\tilde{h}(z)}] \leq[K(w, z)]
$$

Note that by Theorem 3.1.3, $\mathcal{H}_{K}$ consists of analytic functions and hence $\widetilde{h}$ is analytic as well. The uniqueness part follows due to the assumption on the set $\Lambda$ to be determining for $\Omega$.

(ii) Suppose that a positive definite kernel $L(y, x)$ satisfies the condition (3.3). By Theorem 2.1.13 there exists a Hilbert space $\mathcal{H}$ and a function $h: \Lambda \rightarrow \mathcal{H}$ such that $L(w, z)=\left\langle h_{z}, h_{w}\right\rangle_{\mathcal{H}}$ for all $z, w \in \Lambda$. Then we use part (i) for Hilbert space valued functions.

In the successive theorem, we will use the following lemma:

Lemma 3.1.6. Let $\Omega$ be a domain in the complex plane and $f$ either analytic or harmonic in $\Omega$. Then for all $w \in \Omega$ and $\epsilon>0$ such that $D(w ; \epsilon):=\{z \in \mathbb{C}$ : $|z-w| \leq \epsilon\} \subset \Omega$, we have

$$
f(w)=\frac{1}{\pi \epsilon^{2}} \iint_{D(w ; \epsilon)} f(z) d m(z)
$$

where $m(\cdot)$ is the planar Lebesque measure in $\mathbb{C}$.

Proof. By writing $f=u+i v$, it follows that it is sufficient to prove the statement for $f$ harmonic on $\Omega$. Also recall that by the Cauchy integral formula for harmonic functions, for all $r \in[0, \epsilon]$,

$$
f(w)=\frac{1}{2 \pi} \int_{0}^{2 \pi} f\left(w+r e^{i t}\right) d t .
$$

Then by using the change of variables to polar coordinates

$$
x=a+r \cos t, \quad y=b+r \sin t,
$$


where $z=x+i y$ and $w=a+i b$, we have

$$
\begin{aligned}
\frac{1}{\pi \epsilon^{2}} \iint_{D(w, \epsilon)} f(z) d m(z) & =\frac{1}{\pi \epsilon^{2}} \int_{0}^{\epsilon} \int_{0}^{2 \pi} f\left(w+r e^{i t}\right) r d t d r \\
& =\frac{1}{\pi \epsilon^{2}} \int_{0}^{\epsilon}\left(\int_{0}^{2 \pi} f\left(w+r e^{i t}\right) d t\right) r d r \\
& =\frac{1}{\pi \epsilon^{2}} \int_{0}^{\epsilon} 2 \pi f(w) r d r=\left.\frac{2 \pi f(w)}{\pi \epsilon^{2}} \cdot \frac{r^{2}}{2}\right|_{0} ^{\epsilon} \\
& =f(w)
\end{aligned}
$$

Theorem 3.1.7. Let $K(w, z)$ be a locally bounded, sesqui-analytic kernel on $\Omega$. If $K(w, z)$ is positive definite on a determining subset $\Lambda$, then it is on the whole $\Omega$.

Proof. Let $K(w, z)$ be a locally bounded, sesqui-analytic kernel on $\Omega$. Suppose that $K(w, z)$ is positive definite on $\Lambda$. Our aim is to show that $K(w, z)$ is positive definite on the whole $\Omega$. The proof is divided in six steps:

Step 1: $K(w, z)$ is Hermitian on $\Omega$.

Since $K(w, z)$ is positive definite on $\Lambda$, then $K(w, z)=\overline{K(z, w)}$ on $\Lambda$. Then $\overline{K(z, w)}$ will also be sesqui-analytic on $\Lambda$. This implies that $K(w, z)$ and $\overline{K(z, w)}$ are equal on $\Omega$. Hence $K(w, z)$ is Hermitian.

Step 2: There exists a positive Borel function $\rho(z)$ on $\Omega$ which satisfies the following conditions:

(i) $1 / \rho(z)$ is locally bounded.

(ii) $\int_{\Omega}|K(w, z)|^{2} \rho(z) d m(z)<\infty$ for all $w \in \Omega$.

(iii) $\int_{\Omega} \int_{\Omega}|K(w, z)|^{2} \rho(z) \rho(w) d m(z) d m(w)<\infty$

where $m(\cdot)$ denotes the planar Lebesque measure.

Let us write $\Omega$ as an increasing union of bounded subdomains $\left\{\Omega_{n}\right\}_{n \geq 1}$ such that $\overline{\Omega_{n}} \subset \Omega_{n+1}$. 
Let $\sup _{w, z \in \Omega_{n}}|K(w, z)|^{2}=\gamma_{n},(n=1,2,3, \ldots)$. Since $K(w, z)$ is locally bounded, we have $\gamma_{n}<\infty$ for each $n$.

We define $\rho$ as follow,

$$
\rho(z):=\frac{1}{2^{n}\left(\gamma_{n}+1\right) m\left(\Omega_{n} \backslash \Omega_{n-1}\right)} \quad \text { for } \quad z \in \Omega_{n} \backslash \Omega_{n-1} \quad \text { with } \quad \Omega_{0}=\emptyset .
$$

(i) We have $1 / \rho(z)$ is bounded on each $\Omega_{n}$ and every compact subset of $\Omega$ is absorbed in some $\Omega_{m}$. So we get $1 / \rho(z)$ is locally bounded.

(ii) Let $w \in \Omega_{n} \backslash \Omega_{n-1}$. Then

$$
\begin{aligned}
\int_{\Omega}|K(w, z)|^{2} \rho(z) d m(z) & \\
& =\sum_{k=1}^{\infty} \int_{\Omega_{k} \backslash \Omega_{k-1}}|K(w, z)|^{2} \rho(z) d m(z) \\
& \leq \sum_{k=1}^{\infty} \int_{\Omega_{k} \backslash \Omega_{k-1}} \gamma_{k} \rho(z) d m(z) \\
& =\sum_{k=1}^{\infty} \int_{\Omega_{k} \backslash \Omega_{k-1}} \gamma_{k} \frac{1}{2^{k}\left(\gamma_{k}+1\right) m\left(\Omega_{k} \backslash \Omega_{k-1}\right)} d m(z) \\
& =\sum_{k=1}^{\infty} \frac{\gamma_{k}}{2^{k}\left(\gamma_{k}+1\right) m\left(\Omega_{k} \backslash \Omega_{k-1}\right)} \int_{\Omega_{k} \backslash \Omega_{k-1}} d m(z) \\
& \leq \sum_{k=1}^{\infty} 1 / 2^{k}=1<\infty .
\end{aligned}
$$

(iii) To see this we have the following estimations:

$$
\begin{aligned}
\int_{\Omega} \int_{\Omega}|K(w, z)|^{2} \rho(z) \rho(w) d m(z) d m(w) \\
\quad=\int_{\Omega} \rho(w)\left(\int_{\Omega}|K(w, z)|^{2} \rho(z) d m(z)\right) d m(w) \\
\quad=\sum_{k=1}^{\infty} \int_{\Omega_{k} \backslash \Omega_{k-1}} \rho(w)\left(\int_{\Omega}|K(w, z)|^{2} \rho(z) d m(z)\right) d m(w) \\
\leq \sum_{k=1}^{\infty} \int_{\Omega_{k} \backslash \Omega_{k-1}} \rho(w) d m(w) \quad(b y(i i)) \\
=\sum_{k=1}^{\infty} \frac{1}{2^{k}\left(\gamma_{k}+1\right)} \leq 1<\infty
\end{aligned}
$$


Step 3: Define a new measure $d \mu(z):=\rho(z) d m(z)$ on $\Omega$ and let $L^{2}(\Omega, \mu)$ be the associated Hilbert space. Let $A^{2}(\Omega, \mu)$ be the subspace of $L^{2}(\Omega, \mu)$ consisting of all analytic functions in $L^{2}(\Omega, \mu)$. In the following we show that $A^{2}(\Omega, \mu)$ is a reproducing kernel Hilbert space on $\Omega$ and it is closed in $L^{2}(\Omega, \mu)$.

Let us fix $w \in \Omega$ and take $\varepsilon>0$ such that the open disk $D(w, \varepsilon):=\{z$ : $|z-w| \leq \varepsilon\}$ is contained in $\Omega$. For any analytic function $f \in A^{2}(\Omega, \mu)$, according to the previous lemma,

$$
f(w)=\frac{1}{\pi \varepsilon^{2}} \int_{D(w, \varepsilon)} f(z) d m(z) .
$$

Then by the Schwarz Inequality in $L^{2}(\Omega, \mu)$,

$$
\begin{aligned}
|f(w)| & =\left|\frac{1}{\pi \varepsilon^{2}} \int_{D(w, \varepsilon)} f(z) d m(z)\right| \\
& =\frac{1}{\pi \varepsilon^{2}}\left(\int_{\Omega}|f(z)|^{2} \rho(z) d m(z)\right)^{1 / 2}\left(\int_{D(w, \varepsilon)} 1 / \rho(z) d m(z)\right)^{1 / 2} \\
& \leq \kappa\|f\|
\end{aligned}
$$

where $\kappa$ is a finite constant depending on $w$ and $\varepsilon$, but not on $f \in A^{2}(\Omega, \mu)$. Thus, the point evaluation functional $f \longmapsto f(w)$ is continuous on $L^{2}(\Omega, \mu)$ and hence $A^{2}(\Omega, \mu)$ is a reproducing kernel Hilbert space on $\Omega$.

To see that $A^{2}(\Omega, \mu)$ is closed, by the above discussion, since $|f(w)| \leq \kappa\|f\|$, the strong topology of $A^{2}(\Omega, \mu)$ is stronger then the topology of local uniform convergence. This implies that the closure of $A^{2}(\Omega, \mu)$ consists of analytic functions, that is, $A^{2}(\Omega, \mu)$ is closed in $L^{2}(\Omega, \mu)$.

Step 4: Define a linear operator $\mathbf{K}$ in $L^{2}(\Omega, \mu)$ such that

$$
(\mathbf{K} f)(w):=\left\langle f, K_{w}\right\rangle=\int_{\Omega} K(w, z) f(z) d \mu(z)
$$

for all $f \in L^{2}(\Omega, \mu)$ and $w \in \Omega$. We claim that it is unique and bounded.

Note that by (ii), $K_{w}(z)=K(z, w)=\overline{K(w, z)} \in L^{2}(\Omega, \mu)$ and hence $\mathbf{K}$ is 
well-defined. Then by using (iii) and (3.6),

$$
\begin{aligned}
& \int_{\Omega}|(\mathbf{K} f)(w)|^{2} d \mu(w)=\int_{\Omega}\left|\left\langle f, K_{w}\right\rangle\right|^{2} d \mu(w) \\
& \leq \int_{\Omega}\|f\|^{2}\left\|K_{w}\right\|^{2} d \mu(w) \\
& =\|f\|^{2} \int_{\Omega}\left\langle K_{w}, K_{w}\right\rangle d \mu(w) \\
& =\|f\|^{2} \int_{\Omega} K(w, z) K_{w}(z) \rho(z) d m(z) \rho(w) d m(w) \\
& =\|f\|^{2} \int_{\Omega} \int_{\Omega}|K(w, z)|^{2} \rho(z) \rho(w) d m(z) d m(w) \\
& \leq C\|f\|^{2}
\end{aligned}
$$

where $C$ is a finite constant. Hence $\mathbf{K}$ is bounded.

Step $5: \mathbf{K}$ maps $L^{2}(\Omega, \mu)$ in $A^{2}(\Omega, \mu)$.

Note that since the kernel $K$ is Hermitian, it follows that the operator $\mathbf{K}$ is self-adjoint. Since $A^{2}(\Omega, \mu)$ is a closed subspace and all analytic functions in $L^{2}(\Omega, \mu)$ are contained in $A^{2}(\Omega, \mu)$ and for all $w, K_{w}(z)$ is analytic in $z$, it follows that $K_{w} \in A^{2}(\Omega, \mu)$. By Step 4, we have

$$
\left\{K_{w}: w \in \Omega\right\}^{\perp} \subseteq \operatorname{ker}(\mathbf{K})=\left(\operatorname{Ran} \mathbf{K}^{*}\right)^{\perp}=(\operatorname{Ran} \mathbf{K})^{\perp}, \quad \text { as } \mathbf{K}=\mathbf{K}^{*}
$$

Then $(\operatorname{Ran} \mathbf{K})=\left(\operatorname{RanK}^{*}\right) \subset A^{2}(\Omega, \mu)$.

Step 6: Let $L(w, z)$ be the reproducing kernel of $A^{2}(\Omega, \mu)$. Then we have $\left\langle\mathbf{K} L_{z}, L_{w}\right\rangle=K(w, z)$ for all $w, z \in \Omega$.

Let $P$ be the orthogonal projection onto $A^{2}(\Omega, \mu)$. Then for $f \in L^{2}(\Omega, \mu)$, we have $\langle\mathbf{K} f, f\rangle=\langle\mathbf{K} P f, P f\rangle$ since $\operatorname{RanK} \subset A^{2}(\Omega, \mu)$ and $\mathbf{K}$ is self adjoint.

In Step 4 , put $L_{z}$ instead of $f$ with the reproducing property,

$$
\left(\mathbf{K} L_{z}\right)(u)=\left\langle L_{z}, K_{u}\right\rangle=\overline{K_{u}(z)}=\overline{K(z, u)}=K(u, z)=K_{z}(u)
$$

and

$$
\left\langle\mathbf{K} L_{z}, L_{w}\right\rangle=\left\langle K_{z}, L_{w}\right\rangle=\overline{\left\langle L_{w}, K_{z}\right\rangle}=\overline{K_{w}(z)}=K_{z}(w)=K(w, z)
$$


for $w, z \in \Omega$.

Since $\Lambda$ is a determining subset of $\Omega$ and $\left\{L_{z}: z \in \Omega\right\}$ is total in $A^{2}(\Omega, \mu)$, it follows that $\left\{L_{z}: z \in \Lambda\right\}$ is total in $A^{2}(\Omega, \mu)$. Therefore, by (3.8) and taking into account that the linear operator $\mathbf{K}$ is bounded and that the kernel $K$ is positive on $\Lambda$, it follows that the operator $\mathbf{K}$ is positive and hence the kernel $K$ is positive definite on $\Omega$.

Theorem 3.1.8. Suppose that $\Omega^{(1)} \cap \Omega^{(2)} \neq \emptyset$ where $\Omega^{(1)}$ and $\Omega^{(2)}$ are connected domains of the complex plane. If $K^{(1)}(w, z)$ and $K^{(2)}(w, z)$ are locally bounded, sesqui-analytic, positive definite kernels on $\Omega^{(1)}$ and $\Omega^{(2)}$ respectively, such that

$$
K^{(1)}(w, z)=K^{(2)}(w, z) \text { for all } w, z \in \Omega^{(1)} \cap \Omega^{(2)},
$$

then there exists uniquely a locally bounded, sesqui-analytic, positive definite kernel $\tilde{K}(w, z)$ on $\Omega^{(1)} \cup \Omega^{(2)}$ such that

$$
\tilde{K}(w, z)=K^{(i)}(w, z) \text { for } w, z \in \Omega^{(i)}, \quad(i=1,2) .
$$

Proof. Let $\Omega$ be the intersection of $\Omega^{(1)}$ and $\Omega^{(2)}$ and $K(w, z)$ be the restriction of $K^{(1)}(w, z)=K^{(2)}(w, z)$ to this $\Omega$ for $w, z \in \Omega$. So we have

$$
K(w, z)=K^{(1)}(w, z)=K^{(2)}(w, z) \text { for } w, z \in \Omega .
$$

Since $\Omega$ is open in connected $\Omega^{(i)}, \Omega$ is a determining subset of $\Omega^{(1)}$ and $\Omega^{(2)}$. Then, there exists an isometric operator $T^{(i)}$ from $\mathcal{H}_{K^{(i)}}\left(\Omega^{(i)}\right)$ to $\mathcal{H}_{K}(\Omega)$ such that

$$
T^{(i)} K_{z}^{(i)}=K_{z} \text { for } z \in \Omega \quad(i=1,2)
$$

Now we can define the kernel $\tilde{K}(w, z)$ on $\tilde{\Omega}:=\Omega^{(1)} \cup \Omega^{(2)}$ by

$$
\tilde{K}(w, z):=\left\langle T^{(i)} K_{z}^{(i)}, T^{(j)} K_{w}^{(j)}\right\rangle_{K} \text { if } w \in \Omega^{(i)}, z \in \Omega^{(j)} \text { and } i, j \in\{1,2\} .
$$

Then, since $K^{(1)}(w, z)$ and $K^{(2)}(w, z)$ are locally bounded and sesqui-analytic, so is $\tilde{K}(w, z)$.

Moreover by the isometric property of operator $T^{(i)}$, we get from the last equation that

$$
\tilde{K}(w, z)=K^{(i)}(w, z) \text { for } w, z \in \Omega^{(i)} \quad(i=1,2) .
$$


Finally, since $\tilde{K}(w, z)$ is locally bounded and sesqui-analytic on $\tilde{\Omega}$, and $\tilde{K}(w, z)$ is positive definite on the determining subset $\Omega$ of $\tilde{\Omega}$, by Theorem 3.1.7, $\tilde{K}(w, z)$ is positive definite on the whole $\tilde{\Omega}$.

\subsection{Bergman Spaces}

Definition 3.2.1. The space of all analytic functions $f(z)$ on $\Omega$ for which

$$
\iint_{\Omega}|f(z)|^{2} d x d y<\infty, \quad(z=x+i y)
$$

is satisfied, is called the Bergman space on $\Omega$ and denoted by $A^{2}(\Omega)$.

Remark 3.2.2. $A^{2}(\Omega)$ is a reproducing kernel Hilbert space with respect to the inner product

$$
\langle f, g\rangle \equiv\langle f, g\rangle_{\Omega}:=\int_{\Omega} \int f(z) \overline{g(z)} d x d y
$$

and its kernel is called the Bergman kernel of $\Omega$ and denoted by $B^{(\Omega)}(w, z)$.

In the following we will calculate the Bergman kernel for any simply connected domain $\Omega$. Consider the simplest case, that is $\Omega=\mathbb{D}$, where $\mathbb{D}:=\{z:|z|<1\}$. For this case the inner product is

$$
\langle f, g\rangle=\int_{0}^{1} \int_{0}^{2 \pi} f\left(r e^{i \theta}\right) \overline{g\left(r e^{i \theta}\right)} d \theta r d r, \quad\left(z=r e^{i \theta}, r \leq 1\right) .
$$

Theorem 3.2.3. The Bergman kernel for the open unit disc $\mathbb{D}$ is given by

$$
B^{(\mathbb{D})}(w, z)=\frac{1}{\pi} \frac{1}{(1-w \bar{z})^{2}} \quad \text { for } \quad w, z \in \mathbb{D} .
$$

Proof. We divide the proof in three steps.

Step 1: $f_{n}(z)=\sqrt{\frac{n+1}{\pi}} z^{n},(n=0,1,2, \ldots)$ form an orthonormal sequence in $A^{2}(\mathbb{D})$.

Since

$$
\left\langle z^{n}, \overline{z^{m}}\right\rangle=\int_{0}^{1} \int_{0}^{2 \pi} r^{n} e^{i n \theta} r^{m} e^{-i m \theta} d \theta r d r=\frac{2 \pi}{n+m+2} \delta_{n m},
$$


then

$$
\left\langle f_{n}, f_{m}\right\rangle=\delta_{n m}
$$

which means that $\left\{f_{n}(z)\right\}_{n \geq 0}$ forms an orthonormal sequence.

Step 2: $\left\{f_{n}(z)\right\}_{n \geq 0}$ is total in $A^{2}(\mathbb{D})$.

Consider any function $f \in A^{2}(\mathbb{D})$. By the definition of Bergman space, $f$ is analytic in $\mathbb{D}$ and so we have the Taylor series expansion of $f$ as

$$
f(z)=\sum_{0}^{\infty} a_{n} z^{n} .
$$

Then we get

$$
\left\langle f, f_{n}\right\rangle=\sqrt{\frac{\pi}{n+1}} a_{n}, \quad(n=0,1,2, \ldots) .
$$

Therefore, if $f$ is orthogonal to all of $f_{n}$, that is,

$$
\left\langle f, f_{n}\right\rangle=\sqrt{\frac{\pi}{n+1}} a_{n}=0, \quad(n=0,1,2, \ldots)
$$

then $a_{n}=0$ for all $n \geq 0$. In other words, all Taylor coefficients of $f$ vanish and this gives $f=0$. Hence, $\left\{f_{n}\right\}_{n \geq 0}$ is total in $A^{2}(\mathbb{D})$.

Step 3: The Bergman kernel for $\mathbb{D}$ is

$$
B^{(\mathbb{D})}(w, z)=\sum_{0}^{\infty} f_{n}(w) \overline{f_{n}(z)} .
$$

If this is true, then it has to satisfy the reproducing property. Let us check this fact,

$$
\begin{aligned}
\left\langle f, B_{z}^{(\mathbb{D})}\right\rangle & =\left\langle\sum_{0}^{\infty} a_{n} f_{n}(w), \sum_{0}^{\infty} f_{n}(w) \overline{f_{n}(z)}\right\rangle=\sum_{0}^{\infty} f_{n}(z) a_{n}\left\langle f_{n}, f_{n}\right\rangle \\
& =\sum_{0}^{\infty} f_{n}(z) a_{n}=f(z) .
\end{aligned}
$$

Hence, by the uniqueness of the reproducing kernel we have

$$
B^{(\mathbb{D})}(w, z)=\sum_{0}^{\infty} f_{n}(w) \overline{f_{n}(z)} \text { for all } w, z \in \mathbb{D} .
$$


It remains to insert the values of $f_{n}$ and $f_{m}$ into the above equation and get

$$
\begin{aligned}
B^{(\mathbb{D})}(w, z) & =\sum_{n=0}^{\infty} f_{n}(w) \overline{f_{n}(z)}=\sum_{n=0}^{\infty} \sqrt{\frac{n+1}{\pi}} w^{n} \sqrt{\frac{n+1}{\pi}} \overline{z^{n}}=\frac{1}{\pi} \sum_{n=0}^{\infty}(n+1)(w \bar{z})^{n} \\
& =\frac{1}{\pi}\left(1+2 w \bar{z}+3(w \bar{z})^{2}+. .\right)=\left.\frac{1}{\pi} \frac{d}{d \xi}\left(\sum_{n=0}^{\infty} \xi^{n}\right)\right|_{\xi=w \bar{z}} \\
& =\left.\frac{1}{\pi} \frac{1}{(1-\xi)^{2}}\right|_{\xi=w \bar{z}}=\frac{1}{\pi} \frac{1}{(1-w \bar{z})^{2}} .
\end{aligned}
$$

Hence the Bergman kernel for the open unit disc $\mathbb{D}$ is

$$
B^{(\mathbb{D})}(w, z)=\frac{1}{\pi} \frac{1}{(1-w \bar{z})^{2}} \text { for } w, z \in \mathbb{D} .
$$

By the Riemann Mapping Theorem, each simply connected domain (which is not equal to $\mathbb{C}$ ) is mapped conformally onto the open unit disc. Hence we can find the Bergman kernel for an arbitrary simply connected domain $\Omega$, in terms of the associated conformal mapping function. The proof of the following theorem includes the calculation of this kernel.

Theorem 3.2.4. The Bergman kernel of a simply connected domain $\Omega(\neq \mathbb{C})$ is given by

$$
B^{(\Omega)}(w, z)=\frac{1}{\pi} \frac{\phi^{\prime}(w) \overline{\phi^{\prime}(z)}}{(1-\phi(w) \overline{\phi(z)})^{2}} \quad \text { for } \quad w, z \in \Omega,
$$

where $\phi$ is any conformal mapping function from $\Omega$ onto $\mathbb{D}$.

Proof. Let $f \in A^{2}(\mathbb{D})$. Assign $f \longmapsto U f$, where $U$ is the linear mapping on $\Omega$ defined by

$$
(U f)(z)=f(\phi(z)) \phi^{\prime}(z) \text { for } z \in \Omega .
$$

Claim: $U: A^{2}(\mathbb{D}) \longrightarrow A^{2}(\Omega)$ is an isometric operator.

Since we have the Jacobian of $\phi$ as

$$
\left|\phi^{\prime}(z)\right|^{2}=\operatorname{det}\left[\begin{array}{cc}
\frac{\partial}{\partial x} \operatorname{Re} \phi & \frac{\partial}{\partial y} \operatorname{Re} \phi \\
\frac{\partial}{\partial x} \operatorname{Im} \phi & \frac{\partial}{\partial y} \operatorname{Im} \phi
\end{array}\right] \text { where } z=x+i y,
$$


then by the formula of change of variables we have

$$
\begin{aligned}
\iint_{\Omega}|(U f)(z)|^{2} d x d y & =\iint_{\Omega}|f(\phi(z))|^{2}\left|\phi^{\prime}(z)\right|^{2} d x d y, \quad(z=x+i y) \\
& =\iint_{\mathbb{D}}|f(w)|^{2} d u d v, \quad(w=u+i v, \phi(z)=w) .
\end{aligned}
$$

Similarly, take $g \in A^{2}(\Omega)$ and assign $g \longmapsto V g$ where $V$ is the linear mapping on $\Omega$ defined by

$$
(V g)(w)=g(\psi(w)) \cdot \psi^{\prime}(w) \text { for } w \in \mathbb{D}
$$

and $\psi$ is the inverse mapping of $\phi$, i.e. $\psi(w)=z$. By similar arguments, we get that $V$ is also isometric. Since $\phi(\psi(w))=\phi(z)=w$ and $\psi(\phi(z))=\psi(w)=z$, with (3.13) and (3.14) we get $U$ and $V$ are inverse to each other. Thus, since they are both isometric operators, so $U$ is a unitary operator.

For the last part, let us fix $z \in \Omega$. Take $f \in A^{2}(\Omega)$. By using the reproducing property of Bergman kernels $B^{(\Omega)}$ and $B^{(\mathbb{D})}$,

$$
f(\phi(z)) \phi^{\prime}(z)=(U f)(z)=\left\langle U f, B_{z}^{(\Omega)}\right\rangle_{\Omega}=\left\langle f, U^{*} B_{z}^{(\Omega)}\right\rangle_{\mathbb{D}}
$$

and

$$
f(\phi(z)) \phi^{\prime}(z)=\phi^{\prime}(z)\left\langle f, B_{\phi(z)}^{(\mathbb{D})}\right\rangle_{\mathbb{D}}=\left\langle f, \overline{\phi^{\prime}(z)} B_{\phi(z)}^{(\mathbb{D})}\right\rangle_{\mathbb{D}} .
$$

Then, by combining these formulas we get

$$
U^{*} B_{z}^{(\Omega)}=\overline{\phi^{\prime}(z)} B_{\phi(z)}^{(\mathbb{D})},
$$

or equivalently by using the property of $U$ being unitary, we have

$$
B_{z}^{(\Omega)}=U \overline{\phi^{\prime}(z)} B_{\phi(z)}^{(\mathbb{D})} .
$$

It remains to calculate $B^{(\Omega)}(w, z)$ by using (3.11) and (3.15) with unitarity of $U$,

$$
\begin{aligned}
B^{(\Omega)}(w, z) & =\left\langle B_{z}^{(\Omega)}, B_{w}^{(\Omega)}\right\rangle_{\Omega}=\left\langle U \overline{\phi^{\prime}(z)} B_{\phi(z)}^{(\mathbb{D})}, U \overline{\phi^{\prime}(w)} B_{\phi(w)}^{(\mathbb{D})}\right\rangle_{\Omega} \\
& =\phi^{\prime}(w) \cdot \overline{\phi^{\prime}(z)} \cdot\left\langle B_{\phi(z)}^{(\mathbb{D})}, B_{\phi(w)}^{(\mathbb{D})}\right\rangle_{\mathbb{D}} \\
& =\frac{1}{\pi} \cdot \frac{\phi^{\prime}(w) \overline{\phi^{\prime}(z)}}{(1-\phi(w) \overline{\phi(z)})^{2}} \quad \text { for } \quad w, z \in \Omega .
\end{aligned}
$$


The following result is the converse of the previous theorem.

Lemma 3.2.5. A conformal mapping from $\Omega$ to $\mathbb{D}$ can be recaptured from the Bergman kernel of $\Omega$.

Proof. For fixed $z_{0} \in \Omega$, by the Riemann Mapping Theorem, there is a unique analytic function $w=\phi(z)$ mapping $\Omega$ onto the unit disc $\mathbb{D}$ such that

$$
\phi\left(z_{0}\right)=0 \text { and } \phi^{\prime}\left(z_{0}\right)>0 .
$$

By (3.12), for $w \in \Omega$ and $z_{0} \in \Omega$ satisfying (3.16), we find

$$
\phi^{\prime}(w)=\pi \frac{B^{(\Omega)}(w, z)}{\phi^{\prime}\left(z_{0}\right)}
$$

and letting $z_{0}$ instead of $w$ in the last equation, we get

$$
\phi^{\prime}\left(z_{0}\right)=\sqrt{\pi B^{(\Omega)}\left(z_{0}, z_{0}\right)} .
$$

Then if we integrate this equation, we find the conformal mapping function in terms of Bergman kernel, i.e.

$$
\phi(z)=\sqrt{\frac{\pi}{B^{(\Omega)}\left(z_{0}, z_{0}\right)}} \int_{z_{0}}^{z} B^{(\Omega)}\left(w, z_{0}\right) d w .
$$

This completes the proof.

Definition 3.2.6. A Jordan curve is a continuous one-to-one image of $\{|\xi|=1\}$ in $\mathbb{C}$.

Definition 3.2.7. A Green's function $G(w, z)$ of $\Omega$ is a function harmonic in $\Omega$ except at $z$, where it has logarithmic singularity, and continuous in the closure $\bar{\Omega}$, with boundary values $G(w, z)=0$ for all $w \in \partial \Omega$, where $\Omega$ is a finitely connected domain of complex plane.

Suppose now that $\Omega$ is a finitely connected domain in the complex plane, bounded by analytic Jordan curves. $G(w, z)$ has a logarithmic singularity at $z$ means that $G(w, z)-\log \frac{1}{|w-z|}$ is harmonic in a neighborhood of $z$. The symmetry relation $G(w, z)=G(z, w)$ is satisfied for the Green's function. Moreover, the 
Green function is conformally invariant. That is, if $\phi(z)=w$ maps a domain $\mathbb{D}$ conformally onto $\Omega$, and if $G\left(w, z_{0}\right)$ is the Green's function of $\Omega$, then $H(z, \zeta)=$ $G(\phi(z), \phi(\zeta))$ is the Green's function of $\mathbb{D}$. For a simply connected domain $\Omega$, the Green's function is $G(w, z)=-\log |\phi(w)|$, where $\phi$ maps $\Omega$ conformally onto $\mathbb{D}$ and $\phi(z)=0$. In particular, the Green function of $\mathbb{D}$ is

$$
G(w, z)=-\log \left|\frac{w-z}{1-w \bar{z}}\right| .
$$

The following theorem gives the Bergman kernel in terms of the Green's function for the general case.

Theorem 3.2.8. Let $\Omega$ be a finitely connected domain bounded by analytic Jordan curves, and let $G(w, z)$ be the Green's function of $\Omega$. Then the Bergman kernel function is

$$
B^{(\Omega)}(w, z)=-\frac{2}{\pi} \frac{\partial^{2} G}{\partial w \partial \bar{z}}(w, z), \quad w \neq z
$$

Proof. By the definition of the Green's function, we have

$$
G(w, z)=\log \frac{1}{|w-z|}+H(w, z)
$$

in some neighborhood of $z$, where $H(w, z)$ is a harmonic function of $w$. Taking partial derivative with respect to $w$, we get

$$
\frac{\partial G}{\partial w}(w, z)=-\frac{1}{2} \frac{1}{|w-z|}+\frac{\partial H}{\partial w}(w, z)
$$

and now taking partial derivative with respect to $\bar{z}$, since $\frac{\partial^{2}}{\partial w \partial \bar{z}}\left(-\frac{1}{2} \frac{1}{|w-z|}\right)=0$, then we get

$$
\frac{\partial^{2} G}{\partial w \partial \bar{z}}(w, z)=\frac{\partial^{2} H}{\partial w \partial \bar{z}}, \text { for } w \neq z .
$$

Since the boundary curves are analytic and the Green's function vanishes on the boundary, it has a harmonic extension across the boundary. Also, for each $z \in \Omega$, $\frac{\partial G}{\partial w}(w, z)$ is analytic in $w$. Then, $\frac{\partial^{2} G}{\partial w \partial \bar{z}}(w, z)$ is bounded and analytic in $w \in \Omega$, so it belongs to the Bergman space $A^{2}(\Omega)$.

Recall that according to the Cauchy - Green theorem ( see [5] ), we have

$$
\int_{\partial \Omega} F(z) d z=2 i \int_{\Omega} \frac{\partial F}{\partial \bar{z}} d A, \quad \text { for } F \in C^{1}(\bar{\Omega}) .
$$


Suppose $f$ is analytic in $\Omega$ and continuous in $\bar{\Omega}$. Let $\Omega_{\varepsilon}$ be the domain inside $\Omega$ given by a small disc $|w-z| \leq \varepsilon$, and let $\mathbb{T}_{\varepsilon}$ denote the boundary of this disc. Since the Green's function $G(w, z)$ vanishes on the boundary, then $\frac{\partial G}{\partial w}$ also vanishes for $z$ on $\partial \Omega$, then by the Cauchy-Green formula

$$
\frac{1}{2 i} \int_{\mathbb{T}_{\varepsilon}} \frac{\partial G}{\partial w}(w, z) f(z) d z=-\iint_{\Omega_{\varepsilon}} \frac{\partial^{2} G}{\partial w \partial \bar{z}}(w, z) f(z) d A(z),
$$

where the orientation of $\mathbb{T}_{\varepsilon}$ is counterclockwise. However, if we apply the Cauchy Theorem to the left hand side of the above equation,

$$
\int_{\mathbb{T}_{\varepsilon}} \frac{\partial G}{\partial w}(w, z) f(z) d z=\int_{\mathbb{T}_{\varepsilon}}\left(-\frac{1}{2} \frac{1}{|w-z|}+\frac{\partial H}{\partial w}\right) f(z) d z \longrightarrow \pi i f(w) \text { as } \varepsilon \rightarrow 0 .
$$

Hence, applying this result to (3.20) we obtain

$$
\frac{1}{2 i} \pi i f(w)=-\iint_{\Omega} \frac{\partial^{2} G}{\partial w \partial \bar{z}}(w, z) f(z) d A(z) .
$$

Thus we get

$$
f(w)=-\frac{2}{\pi} \iint_{\Omega} \frac{\partial^{2} G}{\partial w \partial \bar{z}}(w, z) f(z) d A(z),
$$

where $f$ is analytic in $\Omega$ and continuous in $\bar{\Omega}$.

Finally, since $\Omega$ has analytic boundary, then the kernel function of $\Omega$ has an analytic continuation across the boundary. Applying this to the function $f(w)=B^{(\Omega)}(w, \zeta)$ we get,

$$
-\frac{2}{\pi} \iint_{\Omega} \frac{\partial^{2} G}{\partial w \partial \bar{z}}(w, z) B^{(\Omega)}(z, \zeta) d A(z)=B^{(\Omega)}(w, \zeta) .
$$

Therefore, by the reproducing property of the kernel function, we get the desired result, i.e.

$$
-\frac{2}{\pi} \iint_{\Omega} \frac{\partial^{2} G}{\partial w \partial \bar{z}}(w, z) B^{(\Omega)}(z, \zeta) d A(z)=-\frac{2}{\pi} \frac{\partial^{2} G}{\partial w \partial \bar{\zeta}}(w, \zeta)=B^{(\Omega)}(w, \zeta) .
$$

\subsection{Szegö Kernel}

Consider the kernels $K_{\alpha}(w, z)$ on the open unit disc $\mathbb{D},(0<\alpha<\infty)$,

$$
K_{\alpha}(w, z):=\frac{1}{(1-w \bar{z})^{\alpha}} \text { for } w, z \in \mathbb{D},
$$


where $\xi^{\alpha}$ is the analytic continuation of $t^{\alpha}$ on $\mathbb{R}_{+}$to the open half plane $\{\xi: \operatorname{Re} \xi>0\}$. By the above definition of kernels, if we have $\alpha=2$, we get $\pi$ times Bergman kernel for $\mathbb{D}$, i.e.

$$
K_{2}(w, z)=\frac{1}{(1-w \bar{z})^{2}}=\pi B^{(\mathbb{D})}(w, z) .
$$

Since $K_{\alpha}(w, z)=\overline{K_{\alpha}(z, w)}$, it is sesqui-analytic and moreover it is locally bounded.

Lemma 3.3.1. The kernel $K_{\alpha}$ defined at (3.21) is positive definite.

Proof. Since

$$
\frac{1}{(1-\xi)^{2}}=\frac{d}{d \xi}\left(\sum_{n=0}^{\infty} \xi^{n}\right), \quad|\xi|<1,
$$

if we take derivatives of both sides $\alpha$ times, it follows that

$$
\begin{aligned}
\frac{1}{(1-\xi)^{\alpha}} & =\frac{1}{(\alpha-1) !} \cdot \frac{d^{\alpha-1}}{d \xi^{\alpha-1}} \cdot\left(\sum_{n=0}^{\infty} \xi^{n}\right) \\
& =\frac{1}{(\alpha-1) !} \sum_{n=0}^{\infty} n(n-1)(n-2)(n-3) \cdots(n-(\alpha-2)) \xi^{(n-(\alpha-1))} \\
& =\frac{1}{(\alpha-1) !} \sum_{N=0}^{\infty}(N+\alpha-1)(N+\alpha-2) \cdots(N+1) \xi^{N} .
\end{aligned}
$$

Let $\Gamma$ be the Gamma function. For $(\alpha=1,2, \ldots)$, we have $\Gamma(\alpha)=(\alpha-1)$ ! and

$$
\frac{\Gamma(n+\alpha)}{\Gamma(n+1)}=(n+\alpha-1)(n+\alpha-2) \cdots(n+1) .
$$

If we put $\xi=w \bar{z}$ into the above equation we obtain

$$
\sum_{n=0}^{\infty} \frac{\Gamma(n+\alpha)}{\Gamma(\alpha) \Gamma(n+1)}(w \bar{z})^{n}=\frac{1}{(1-w \bar{z})^{\alpha}} \quad \text { for } w, z \in \mathbb{D}, \quad(\alpha=1,2, \ldots) .
$$

We can generalize this result for each $0<\alpha<\infty$, so that

$$
\sum_{n=0}^{\infty} \frac{\Gamma(n+\alpha)}{\Gamma(\alpha) \Gamma(n+1)}(w \bar{z})^{n}=\frac{1}{(1-w \bar{z})^{\alpha}}=K_{\alpha}(w, z) \quad \text { for } \quad w, z \in \mathbb{D} .
$$

Therefore, since $\left[w \bar{z}^{n}\right]$ is positive definite for each $n$ and

$$
\frac{\Gamma(n+\alpha)}{\Gamma(\alpha) \Gamma(n+1)}>0, \quad(n=0,1,2, \ldots),
$$

then $K_{\alpha}(w, z)$ is positive definite. 
Theorem 3.3.2. (i) The Hilbert space $\mathcal{H}_{K_{\alpha}}(\mathbb{D})$ coincides with the space of analytic functions $f(z)=\sum_{0}^{\infty} a_{n} z^{n}$ on $\mathbb{D}$ such that

$$
\sum_{n=0}^{\infty} \frac{\Gamma(\alpha) \Gamma(n+1)}{\Gamma(n+\alpha)}\left|a_{n}\right|^{2}<\infty
$$

equipped with the inner product

$$
\langle g, h\rangle=\sum_{0}^{\infty} \frac{\Gamma(\alpha) \Gamma(n+1)}{\Gamma(n+\alpha)} b_{n} \bar{c}_{n}
$$

for $g(z)=\sum_{0}^{\infty} b_{n} z^{n}$ and $h(z)=\sum_{0}^{\infty} c_{n} z^{n}$.

(ii) When $\alpha>1, \mathcal{H}_{K_{\alpha}}(\mathbb{D})$ also coincides with the space of analytic functions $f(z)=\sum_{n=0}^{\infty} a_{n} z^{n}$ such that

$$
\iint_{\mathbb{D}}\left(1-|z|^{2}\right)^{\alpha-2}|f(z)|^{2} d x d y<\infty, \quad(z=x+i y)
$$

equipped with the inner product

$$
\langle g, h\rangle=\frac{\alpha-1}{\pi} \iint_{\mathbb{D}}\left(1-|z|^{2}\right)^{\alpha-2} \mid g(z) \overline{|| h(z) \mid} d x d y .
$$

Proof. (i) Since $f(z)=\sum_{0}^{\infty} a_{n} z^{n}$, then

$$
\begin{aligned}
|f(z)| & =\left|\sum_{n=0}^{\infty} a_{n} z^{n}\right| \leq \sum_{n=0}^{\infty}\left|a_{n}\right||z|^{n} \\
& \leq\left(\sum_{n=0}^{\infty} \frac{\Gamma(\alpha) \Gamma(n+1)}{\Gamma(n+\alpha)}\left|a_{n}\right|^{2}\right)^{1 / 2}\left(\sum_{n=0}^{\infty} \frac{\Gamma(n+\alpha)}{\Gamma(\alpha) \Gamma(n+1)}|z|^{2 n}\right)^{1 / 2},
\end{aligned}
$$

and with the inner product (3.23), strong topology is stronger than the topology of local uniform convergence on $\mathbb{D}$. Hence this space of analytic functions becomes a reproducing kernel Hilbert space on $\mathbb{D}$.

The construction of the kernel will be similar as the construction of Bergman kernel.

Step 1: $\quad f_{n}(z):=\sqrt{\frac{\Gamma(\alpha+n)}{\Gamma(\alpha) \Gamma(n+1)}} z^{n}, \quad(n=0,1,2, \ldots)$, form an orthonormal sequence.

$$
\begin{aligned}
\left\langle f_{n}, f_{m}\right\rangle & =\left\langle\sqrt{\frac{\Gamma(\alpha+n)}{\Gamma(\alpha) \Gamma(n+1)}} z^{n}, \sqrt{\frac{\Gamma(\alpha+n)}{\Gamma(\alpha) \Gamma(n+1)}} z^{m}\right\rangle \\
& =\delta_{n m}, \quad \text { for } n, m=0,1, \ldots
\end{aligned}
$$


Thus, $\left\{f_{n}\right\}_{n \geq 0}$ is an orthonormal sequence.

Step 2: $\left\{f_{n}\right\}_{n \geq 0}$ is complete.

Consider the inner product in (3.23) of $f_{n}(z)$ with $f=\sum_{n=0}^{\infty} a_{k} z^{k}$,

$$
\left\langle f, f_{n}\right\rangle=a_{n} \sqrt{\frac{\Gamma(\alpha) \Gamma(n+1)}{\Gamma(n+\alpha)}}, \quad(n=0,1,2, \ldots) .
$$

Therefore, $f$ is orthogonal to all of $f_{n}$, that is

$$
\left\langle f, f_{n}\right\rangle=a_{n} \sqrt{\frac{\Gamma(\alpha) \Gamma(n+1)}{\Gamma(n+\alpha)}}=0, \quad(n=0,1,2, \ldots)
$$

only if $a_{n}=0$ for each $n$. Thus $f=0$ and $f_{n}$ is total.

Step 3: $K_{\alpha}(w, z)=\sum_{n=0}^{\infty} f_{n}(w) \overline{f_{n}(z)}$.

To prove this, we verify the reproducing property:

$$
\begin{aligned}
\left\langle f, K_{z}\right\rangle & =\left\langle\sum_{n=0}^{\infty} a_{n} f_{n}(w), \sum_{n=0}^{\infty} f_{n}(w) f_{n}(z)\right\rangle \\
& =\sum_{n=0}^{\infty} a_{n} f_{n}(z)=f(z) .
\end{aligned}
$$

Hence, $K_{\alpha}(w, z)=\sum_{n=0}^{\infty} f_{n}(w) \overline{f_{n}(z)}$.

Finally, inserting the values of $f_{n}(w)$ and $f_{n}(z)$ in this equation, we get

$$
\begin{aligned}
K_{\alpha}(w, z) & =\sum_{n=0}^{\infty} f_{n}(w) \overline{f_{n}(z)}=\sum_{n=0}^{\infty} \sqrt{\frac{\Gamma(\alpha+n)}{\Gamma(\alpha) \Gamma(n+1)}} w^{n} \sqrt{\frac{\Gamma(\alpha+n)}{\Gamma(\alpha) \Gamma(n+1)}} \bar{z}^{n} \\
& =\sum_{n=0}^{\infty} \frac{\Gamma(\alpha+n)}{\Gamma(\alpha) \Gamma(n+1)}(w \bar{z})^{n}=\frac{1}{(1-w \bar{z})^{\alpha}} .
\end{aligned}
$$

(ii) We change the coordinates to polar coordinates. For $f=\sum_{n=0}^{\infty} a_{n} z^{n}$, 
consider the following integration,

$$
\begin{aligned}
\frac{\alpha-1}{\pi} \iint_{\mathbb{D}} & \left(1-|z|^{2}\right)^{\alpha-2}|f(z)|^{2} d x d y \\
& =\frac{\alpha-1}{\pi} \int_{0}^{1} \int_{0}^{2 \pi}\left(1-r^{2}\right)^{\alpha-2}\left|\sum_{n=0}^{\infty} a_{n}\left(r e^{i \theta}\right)^{n}\right|^{2} d \theta r d r \quad\left(z=r e^{i \theta}\right) \\
& =\frac{\alpha-1}{\pi} \int_{0}^{1} \int_{0}^{2 \pi}\left(1-r^{2}\right)^{\alpha-2}\left(\sum_{n, m \geq 0} a_{m} \bar{a}_{n} r^{m} r^{m} e^{i \theta(m-n)}\right) d \theta r d r \\
& =\frac{\alpha-1}{\pi} \sum_{n, m \geq 0} a_{m} \bar{a}_{n} \int_{0}^{1} \int_{0}^{2 \pi}\left(1-r^{2}\right)^{\alpha-2} r^{m+n+1} e^{i \theta(m-n)} d \theta d r \\
& =\frac{\alpha-1}{\pi} \sum_{n=0}^{\infty}\left|a_{n}\right|^{2} \int_{0}^{1} 2 \pi\left(1-r^{2}\right)^{\alpha-2} r^{2 n+1} d \theta d r \\
& =2(\alpha-1) \sum_{n=0}^{\infty}\left|a_{n}\right|^{2} \int_{0}^{1} 2 \pi\left(1-r^{2}\right)^{\alpha-2} r^{2 n+1} d \theta d r \quad\left(t=r^{2}, d t=2 r d r\right) \\
& =(\alpha-1) \sum_{n=0}^{\infty}\left|a_{n}\right|^{2} \int_{0}^{1}(1-t)^{\alpha-2} t^{n} d t .
\end{aligned}
$$

Since

$$
\int_{0}^{1}(1-t)^{\beta-1} t^{\gamma-1} d t=\frac{\Gamma(\beta) \Gamma(\gamma)}{\Gamma(\beta+\gamma)}, \quad \text { for } \beta, \gamma>0
$$

then

$$
(\alpha-1) \int_{0}^{1}(1-t)^{\alpha-2} t^{n} d t=(\alpha-1) \frac{\Gamma(\alpha-1) \Gamma(n+1)}{\Gamma(n+\alpha)}=\frac{\Gamma(\alpha) \Gamma(n+1)}{\Gamma(n+\alpha)} .
$$

Inserting this in the above integration, we get

$$
(\alpha-1) \sum_{0}^{\infty}\left|a_{n}\right|^{2} \int_{0}^{1}(1-t)^{\alpha-2} t^{n} d t=\sum_{0}^{\infty} \frac{\Gamma(\alpha) \Gamma(n+1)}{\Gamma(n+\alpha)}\left|a_{n}\right|^{2}
$$

or equivalently,

$$
\frac{\alpha-1}{\pi} \iint_{\mathbb{D}}\left(1-|z|^{2}\right)^{\alpha-2}|f(z)|^{2} d x d y=\sum_{0}^{\infty} \frac{\Gamma(\alpha) \Gamma(n+1)}{\Gamma(n+\alpha)}\left|a_{n}\right|^{2} .
$$

Hence, the last equality shows that on the space of analytic functions, the two inner products (3.23) and (3.24) are the same. 
According to the values that $\alpha$ takes, the behavior of $\mathcal{H}_{K_{\alpha}}(\mathbb{D})$ changes. If $\alpha>1, \mathcal{H}_{K_{\alpha}}(\mathbb{D})$ becomes a Hilbert space of analytic functions $f(z)$ on $\mathbb{D}$ such that

$$
\int_{\mathbb{D}}|f(z)|^{2} d \mu_{\alpha}(z)<\infty
$$

where $\mu_{\alpha}$ is the measure on $\mathbb{D}$ given by

$$
d \mu_{\alpha}(z)=\frac{\alpha-1}{\pi}\left(1-|z|^{2}\right)^{\alpha-2} d x d y,(z=x+i y) .
$$

Definition 3.3.3. For $\alpha>1, \mathcal{H}_{K_{\alpha}}(\mathbb{D})$ is called the weighted Bergman space on $\mathbb{D}$ (with weight $\frac{\alpha-1}{\pi}\left(1-|z|^{2}\right)^{\alpha-2}$ ).

Lemma 3.3.4. If $\alpha<1, \mathcal{H}_{K_{\alpha}}(\mathbb{D})$ is not a reproducing kernel Hilbert space with respect to the inner product (3.24).

Proof. Suppose that for $\alpha<1, \mathcal{H}_{K_{\alpha}}(\mathbb{D})$ is a reproducing kernel space. Let $\phi(z)$ be an analytic function such that $|\phi(z)| \leq 1$ for $z \in \mathbb{D}$. Then $f \longmapsto \phi \cdot f$ defines a continuous linear operator on $\mathcal{H}_{K_{\alpha}}(\mathbb{D})$ such that

$$
\|\phi f\|_{K_{\alpha}} \leq\|f\|_{K_{\alpha}}, \quad \text { where } f \in \mathcal{H}_{K_{\alpha}}(\mathbb{D}) .
$$

Then, in terms of the kernel $K_{\alpha}(w, z)$, inequality (3.25) becomes

$$
\left[\phi(w) K_{\alpha}(w, z) \overline{\phi(z)}\right] \leq\left[K_{\alpha}(w, z)\right] \text { on } \mathbb{D}
$$

and hence we get

$$
\left[K_{\alpha}(w, z)(1-\phi(w) \overline{\phi(z)})\right] \geq 0 \text { on } \mathbb{D} .
$$

This implies that, for any $z \in \mathbb{D}$, the following $2 \times 2$ matrix is positive definite

$$
\left[\begin{array}{ll}
K_{\alpha}(0,0)\left(1-|\phi(0)|^{2}\right) & K_{\alpha}(0, z)(1-\phi(0) \overline{\phi(z)}) \\
K_{\alpha}(z, 0)(1-\phi(z) \overline{\phi(0)}) & K_{\alpha}(z, z)\left(1-|\phi(z)|^{2}\right)
\end{array}\right] \geq 0 .
$$

If $\phi(0)=0$, then (3.26) becomes

$$
\left[\begin{array}{ll}
K_{\alpha}(0,0) & K_{\alpha}(0, z) \\
K_{\alpha}(z, 0) & K_{\alpha}(z, z)\left(1-|\phi(z)|^{2}\right)
\end{array}\right]=\left[\begin{array}{ll}
1 & 1 \\
1 & \frac{1}{\left(1-|z|^{2}\right)^{\alpha}}\left(1-|\phi(z)|^{2}\right)
\end{array}\right] \geq 0
$$


where $K_{\alpha}(w, z)=1 /(1-w \bar{z})^{\alpha}$. This implies

$$
\frac{1-|\phi(z)|^{2}}{\left(1-|z|^{2}\right)^{\alpha}}-1 \geq 0 \text { and } 1-\left(1-|z|^{2}\right)^{\alpha} \geq|\phi(z)|^{2} .
$$

Then, for $0 \neq z \in \mathbb{D}$,

$$
\frac{1-\left(1-|z|^{2}\right)^{\alpha}}{|z|^{2}} \geq \frac{|\phi(z)|^{2}}{|z|^{2}} .
$$

Now consider the analytic function $\phi_{t}(z):=z(z-t) /(1-t z),(0<t<1)$, which satisfies $\phi_{t}(0)=0$ and $\left|\phi_{t}(z)\right| \leq 1$ for $z \in \mathbb{D}$ and fix $z_{0} \in \mathbb{D}$. Since we have $\alpha<1$, then

$$
\left(1-\left|z_{0}\right|^{2}\right)^{\alpha}>1-\left|z_{0}\right|^{2}
$$

and by this, we get the following

$$
1>\frac{1-\left(1-\left|z_{0}\right|^{2}\right)^{\alpha}}{\left|z_{0}\right|^{2}} .
$$

Then, if we take the limit of $\left|\phi_{t}\left(z_{0}\right) / z_{0}\right|^{2}$ as $t \longrightarrow 1$,

$$
\lim _{t \rightarrow 1}\left|\phi_{t}\left(z_{0}\right) / z_{0}\right|^{2}=\lim _{t \rightarrow 1}\left|\frac{z_{0}\left(z_{0}-t\right)}{\left(1-t z_{0}\right) z_{0}}\right|^{2}=\lim _{t \rightarrow 1}\left|\frac{z_{0}-t}{1-t z_{0}}\right|^{2}=1 .
$$

But this contradicts with the inequality (3.27). Hence, for $\alpha<1, \mathcal{H}_{K_{\alpha}}(\mathbb{D})$ is not a reproducing kernel Hilbert space with respect to the inner product (3.24).

It remains the case when $\alpha=1$. Let us denote by $\mathbb{T}$ the boundary of $\mathbb{D}$, i.e. $\mathbb{T}=\{\xi:|\xi|=1\}$ and let $\sigma$ be the normalized arc-length measure such that

$$
d \sigma(\xi):=\frac{1}{2 \pi}|d \xi| \equiv \frac{1}{2 \pi} d \theta, \quad\left(\xi=e^{i \theta}\right) .
$$

Consider the Hilbert space $L^{2}(\mathbb{T}) \equiv L^{2}(\mathbb{T}, \sigma)$ of measurable functions on $\mathbb{T}$. We have the inner product

$$
\langle f, g\rangle_{L^{2}}:=\int_{\mathbb{T}} f(\xi) \overline{g(\xi)} d \sigma(\xi),
$$

with respect to which the functions $\phi_{n}(\xi):=\xi^{n},(n \in \mathbb{Z})$, which form a complete orthonormal sequence.

Definition 3.3.5. The closed linear span of $\left\{\phi_{n}: n=0,1, \cdots\right\}$ is called the Hardy space on $\mathbb{T}$ and is denoted by $H^{2}(\mathbb{T})$. 
By Corollary A.2.3, $f \in L^{2}(\mathbb{T})$ belongs to Hardy space $H^{2}(\mathbb{T})$ if and only if it is orthonormal to all $\phi_{n}(n<0$, ) i.e. all Fourier coefficients of $f$ which have negative indices vanish. Then we have

$$
\langle f, g\rangle_{L^{2}}=\sum_{n=0}^{\infty} a_{n} \bar{b}_{n} \text { for } f, g \in H^{2}(\mathbb{T}),
$$

where

$$
a_{n}=\left\langle f, \phi_{n}\right\rangle_{L^{2}} \text { and } b_{n}=\left\langle g, \phi_{n}\right\rangle_{L^{2}} \quad(n=0,1, \ldots) .
$$

Before stating the last theorem of this chapter, we will define the Poisson kernel and some important properties of this kernel.

Definition 3.3.6. For $z \in \mathbb{D}$ and $\xi \in \mathbb{T}, P_{z}(\xi)$ is called the Poisson kernel and defined by

$$
P_{z}(\xi):=\frac{1-|z|^{2}}{|1-\xi \bar{z}|^{2}}
$$

Equivalently, for $z=r e^{i \theta}(0 \leq r<1)$ and $\xi=e^{i t}(-\infty<t<\infty)$, the Poisson kernel can be written as

$$
P_{r}(t):=\frac{1-r^{2}}{1-2 r \cos t+r^{2}}=\sum_{-\infty}^{\infty} r^{|n|} e^{i n t}
$$

We recall some properties of the Poisson kernel, (e.g. see [7]):

(i) $P_{r}(t) \geq 0$, for all $r<1$.

(ii) $P_{r}(t+2 \pi)=P_{r}(t)$

(iii) $\int_{-\pi}^{\pi} P_{r}(t) d t=2 \pi$, for all $r<1$.

(iv) Given $\delta>0, P_{r}(t) \rightarrow 0$ uniformly for $\delta \leq|t| \leq \pi$ as $r \rightarrow 1$.

Theorem 3.3.7. The correspondence

$$
f \longmapsto \tilde{f}(z):=\sum_{n=0}^{\infty} a_{n} z^{n} \quad \text { with } a_{n}=\left\langle f, \phi_{n}\right\rangle_{L^{2}}, \quad(n=0,1, \ldots)
$$

yields a unitary operator $U$ from $H^{2}(\mathbb{T})$ onto $H_{K_{1}}(\mathbb{D})$. Conversely, $f$ can be recaptured from $\tilde{f}(z)$ by the formula

$$
f(\xi)=\lim _{r \rightarrow 1} \tilde{f}(r \xi) \text { for almost all } \xi \in \mathbb{T} .
$$


Proof. Put $\alpha=1$ in $\frac{\Gamma(\alpha) \Gamma(n+1)}{\Gamma(n+\alpha)}$. We get $\frac{\Gamma(1) \Gamma(n+1)}{\Gamma(n+1)}=1$.

Then (3.23) in Theorem 3.3 .2 becomes

$$
\langle g, h\rangle=\sum_{0}^{\infty} b_{n} \bar{c}_{n}, \text { for } g(z)=\sum_{0}^{\infty} b_{n} z^{n} \text { and } h(z)=\sum_{0}^{\infty} c_{n} z^{n} .
$$

In other words, equation (3.23) becomes the same as the equation (3.29). This implies that the operator $U$ from $H^{2}(\mathbb{T})$ onto $H_{K_{1}}(\mathbb{D})$, is isometric on the linear span of $\left\{\phi_{n}: n=0,1, \ldots\right\}$. Since $\left\{\phi_{n}: n=0,1, \ldots\right\}$ and $\left\{\tilde{\phi}_{n}: n=0,1, \ldots\right\}$ are total in $H^{2}(\mathbb{T})$ and $H_{K_{1}}(\mathbb{D})$, respectively, then $U$ is unitary. Now by (3.29),

$$
\begin{aligned}
\langle\tilde{f}(r \xi)-f(\xi), \tilde{f}(r \xi)-f(\xi)\rangle & =\int_{\mathbb{T}}|\tilde{f}(r \xi)-f(\xi)|^{2} d \sigma(\xi) \\
& =\sum_{0}^{\infty}\left|a_{n}\right|^{2}\left(1-r^{n}\right)^{2} \longrightarrow 0 \text { as } r \rightarrow 1 .
\end{aligned}
$$

Therefore, $\tilde{f}(r \xi)$ converges to $f(\xi)$ in measure. It remains to show that the convergence is almost everywhere. Let us define a kernel $S_{z}(\xi)$ as

$$
S_{z}(\xi):=\frac{1}{1-\xi \bar{z}} \text { for } \xi \in \mathbb{T} .
$$

Then we have

$$
\tilde{f}(z)=\left\langle f, S_{z}\right\rangle_{L^{2}} \quad \text { for } z \in \mathbb{D}, f \in H^{2}(\mathbb{T}) .
$$

Consider the sum

$$
\begin{aligned}
S_{z}(\xi)+\overline{S_{z}(\xi)}-1 & =\frac{1}{1-\xi \bar{z}}+\frac{1}{1-z \bar{\xi}}-1, \quad\left(z=r e^{i \theta}\right), \quad\left(\xi=e^{i t}\right) \\
& =\frac{1}{1-e^{i t} \overline{r e} e^{i \theta}}+\frac{1}{1-r e^{i \theta} \overline{e^{i t}}}-1 \\
& =\frac{1-r^{2}}{1-2 r \cos (t-\theta)+r^{2}} \\
& =P_{r}(t-\theta)
\end{aligned}
$$

and by

$$
P_{z}(\xi)=\frac{1-|z|^{2}}{|1-\xi \bar{z}|^{2}}=\frac{1-r^{2}}{1-2 r \cos (t-\theta)+r^{2}}=P_{r}(t-\theta), \quad\left(z=r e^{i \theta}\right), \quad\left(\xi=e^{i \theta}\right)
$$

we get

$$
P_{z}(\xi)=S_{z}(\xi)+\overline{S_{z}(\xi)}-1
$$


Then consider the inner product $\left\langle f, P_{z}\right\rangle$. Since $\overline{S_{z}(\xi)}-1$ is orthogonal to $f$ in $L^{2}(\mathbb{T})$, we get

$$
\begin{aligned}
\left\langle f, P_{z}\right\rangle_{L^{2}} & =\left\langle f, S_{z}(\xi)+\overline{S_{z}(\xi)}-1\right\rangle_{L^{2}}=\left\langle f, S_{z}(\xi)\right\rangle+\left\langle f, \overline{S_{z}(\xi)}-1\right\rangle_{L^{2}} \\
& =\left\langle f, S_{z}(\xi)\right\rangle_{L^{2}}=\tilde{f}(z) .
\end{aligned}
$$

So, instead of (3.31), we can use $\tilde{f}(z)=\left\langle f, P_{z}\right\rangle_{L^{2}}$. Then, by the definition of the inner product in $L^{2}$, we have

$$
\tilde{f}(z)=\left\langle f, P_{z}\right\rangle_{L^{2}}=\int_{-\pi}^{\pi} f(\xi) \overline{P_{z}(\xi)} d \sigma(\xi) .
$$

Changing variables as $z=r e^{i \theta}$ and $\xi=e^{i \theta}$, it follows

$$
\tilde{f}\left(r e^{i \theta}\right)=\frac{1}{2 \pi} \int_{-\pi}^{\pi} f\left(e^{i \theta}\right) P_{r}(t-\theta) d t=\frac{1}{2 \pi} \int_{-\pi}^{\pi} f\left(e^{i(t+\theta)}\right) P_{r}(t) d t .
$$

We fix $\theta$ in $(-\pi, \pi)$ so that $F^{\prime}(\theta)$ exists, where $F(\theta):=\int_{-\pi}^{\pi} f\left(e^{i \theta}\right) d t$. By using the property (iii) of the Poisson kernel,

$$
\tilde{f}\left(r e^{i \theta}\right)-F^{\prime}(\theta)=\frac{1}{2 \pi} \int_{\pi}^{\pi}\left(\tilde{f}\left(r e^{i \theta}\right)-F^{\prime}(\theta)\right) P_{r}(t) d t .
$$

We make change of variables and integrate by parts the right hand side of this equation, say $u=P_{r}(t)$ and $d v=\left(\tilde{f}\left(r e^{i \theta}\right)-F^{\prime}(\theta)\right) d t$ and get

$$
\frac{1}{2 \pi}\left[\left(F(\theta+t)-F^{\prime}(\theta) t\right) P_{r}(t)\right]_{-\pi}^{\pi}-\frac{1}{2 \pi} \int_{-\pi}^{\pi}\left(F(\theta+t)-F^{\prime}(\theta) t\right) P_{r}^{\prime}(t) d t .
$$

Then the first term converges to 0 as $r \rightarrow 1$. So, we have

$$
\lim _{r \rightarrow 1} \tilde{f}\left(r e^{i \theta}\right)-F^{\prime}(\theta)=\lim _{r \rightarrow 1} \frac{1}{2 \pi} \int_{-\pi}^{\pi}\left(F(\theta+t)-F^{\prime}(\theta) t\right) P_{r}^{\prime}(t) d t .
$$

For any $\varepsilon>0$ there exists $\delta>0$ such that

$$
\left|F(\theta+t)-F(\theta-t)-2 F^{\prime}(\theta) t\right| \leq \varepsilon t \quad \text { for } 0 \leq t \leq \delta .
$$

On the other hand,

$$
\begin{aligned}
\tilde{f}\left(r e^{i \theta}\right)-F^{\prime}(\theta) & =\frac{1}{2 \pi} \int_{-\pi}^{\pi}\left(F(\theta+t)-F^{\prime}(\theta) t\right) P_{r}^{\prime}(t) d t \\
& \leq \frac{1}{2 \pi} \int_{0}^{\delta}\left(F(\theta+t)-F(\theta-t)-2 F^{\prime}(\theta) t\right) P_{r}^{\prime}(t) d t \\
& +\frac{1}{2 \pi} \int_{\delta \leq|t| \leq \pi}\left(F(\theta+t)-F^{\prime}(\theta) t\right) P_{r}^{\prime}(t) d t,
\end{aligned}
$$


and then by using (3.35),

$$
\left|\frac{1}{2 \pi} \int_{-\pi}^{\pi}\left(F(\theta+t)-F^{\prime}(\theta) t\right) P_{r}^{\prime}(t) d t\right| \leq \varepsilon \int_{0}^{\pi} t\left|P_{r}^{\prime}(t)\right| d t+\gamma \sup _{\delta \leq|t| \leq \pi}\left|P_{r}^{\prime}(t)\right|,
$$

where

$$
\gamma=\sup _{|t| \leq \pi}\left|F(\theta+t)-F^{\prime}(\theta) t\right|
$$

Now, since we have the derivative of $P_{r}(t)$ as

$$
P_{r}^{\prime}(t)=\frac{2 r \sin t\left(1-r^{2}\right)}{\left(1-2 r \cos t+r^{2}\right)^{2}}
$$

taking modulus of this derivative we get

$$
\left|P_{r}^{\prime}(t)\right|=\left|\frac{2 r \sin t\left(1-r^{2}\right)}{\left(1-2 r \cos t+r^{2}\right)^{2}}\right| \leq \frac{2 r\left(1-r^{2}\right)}{\left(1-2 r \cos t+r^{2}\right)^{2}},
$$

and this implies $\sup _{\delta \leq|t| \leq \pi}\left|P_{r}^{\prime}(t)\right| \longrightarrow 0$, as $r \rightarrow 1$. Also, $t\left|P_{r}^{\prime}(t)\right|$ will be clearly integrable on $[0, \pi]$. Consequently, taking the limsup of (3.34), it converges to 0 as $r \rightarrow 1$. That is

$$
\limsup _{r \rightarrow 1}\left|\tilde{f}\left(r e^{i \theta}\right)-F^{\prime}(\theta)\right|=0 .
$$

Hence $\tilde{f}\left(r e^{i \theta}\right)$ converges as $r \rightarrow 1$ at all $\theta$ for which $F^{\prime}(\theta)$ exists.

Since $\tilde{f}(z)$ is analytic on $\mathbb{D}$ and $f(\xi)$ is its boundary function on $\mathbb{T}$, then $\tilde{f}(z)$ is the analytic extension of $f$ to $\mathbb{D}$.

Definition 3.3.8. The kernel

$$
S(\xi, z):=\frac{1}{1-\xi \bar{z}} \text { for } \xi \in \mathbb{T} \text { and } z \in \mathbb{D},
$$

or its analytic extension

$$
\tilde{S}(w, z):=\frac{1}{1-w \bar{z}} \text { for } w, z \in \mathbb{D} .
$$

is called the Szegö kernel .

Since $K_{\alpha}(w, z)=\frac{1}{(1-w \bar{z})^{\alpha}}$, note that it is equal to the Szegö kernel for $\alpha=1$, i.e. $\tilde{S}(w, z)=K_{1}(w, z)$.

We will end this chapter by a remark. 
Remark 3.3.9. The orthogonal projection $P_{H^{2}(\mathbb{T})}$ from $L^{2}(\mathbb{T})$ to the Hardy space $H^{2}(\mathbb{T})$ can be expressed in terms of the Szegö kernel as follows:

$$
\left(P_{H^{2}} g\right)(z)=\left\langle g, S_{z}\right\rangle_{L^{2}} \text { for } z \in \mathbb{D} \text { and } g \in L^{2}(\mathbb{T}) \text {. }
$$

Moreover, by Theorem 3.3.7, we have

$$
\left(P_{H^{2}} g\right)(\xi)=\lim _{r \rightarrow 1}\left\langle g, S_{r \xi}\right\rangle_{L^{2}} \text { for almost all } \xi \in \mathbb{T} \text {. }
$$




\section{Chapter 4}

\section{Interpolation Theorems of Nevanlinna-Pick Type}

\subsection{General definition of Hardy spaces}

In the previous chapter we have defined $H^{p}$ spaces for the case $p=2$. Before giving a more general definition and some properties of $H^{p}$ spaces, we first define the integral means.

Definition 4.1.1. For a function analytic in the unit disc $\mathbb{D}$, the integral means are defined by

$$
M_{p}(r, \psi)=\left(\frac{1}{2 \pi} \int_{0}^{2 \pi}\left|\psi\left(r e^{i \theta}\right)\right|^{p} d \theta\right)^{1 / p}, \quad(0<p<\infty)
$$

and

$$
M_{\infty}(r, \psi)=\max _{0 \leq \theta<2 \pi}\left|\psi\left(r e^{i \theta}\right)\right| .
$$

Definition 4.1.2. The class $H^{p}$ is the set of all functions analytic in $\mathbb{D}$ for which the integral means $M_{p}(r, \psi)$ are bounded for $0 \leq r<1$.

By the above definitions and the maximum modulus principle, if $M_{p}(r, \psi)$ stays bounded as $r \rightarrow 1$, then $\psi$ is said to belong to the $H^{p}$ space. 
Consider the case $p=2$. If $\psi(z)=\sum a_{n} z^{n}$ is analytic in $\mathbb{D}$, then by Parseval's identity, we have

$$
M_{2}^{2}(r, \psi)=\sum_{n=0}^{\infty}\left|a_{n}\right|^{2} r^{2 n} .
$$

This shows that $M_{2}(r, \psi)$ increases with $r$, and that $\psi \in H^{2}$ if and only if $\sum\left|a_{n}\right|^{2}<\infty$. Similarly, it follows from the maximum modulus principle that $M_{\infty}$ increases with $r$. This situation is more complicated for the other values of $p$, but $M_{p}(r, \psi)$ is always a non-decreasing function.

The $H^{p}$ class is a linear space under addition and scalar multiplication. The norm $\|\psi\|_{H^{p}}$ of a function $\psi \in H^{p}$ is defined as the limit of $M_{p}(r, \psi)$ as $r \rightarrow 1$ and it is a true norm if $p \geq 1$.

Another property of $H^{p}$ spaces is that, if $0<p<q \leq \infty$, then $H^{p} \supset H^{q}$.

Moreover, by the above relations, $H^{\infty}(\mathbb{D})$ is the class of all bounded analytic functions $\psi(z)$ on $\mathbb{D}$ with norm

$$
\|\psi\|_{\infty}=\sup _{z \in \mathbb{D}}|\psi(z)|
$$

According to Theorem 3.3.7, each $\psi \in H^{\infty}(\mathbb{D})$ admits the boundary value for $\sigma$-almost all $\zeta \in \mathbb{T}, \quad(\mathbb{T}$ is the unit circle),

$$
\psi(\zeta):=\lim _{r \rightarrow 1} \psi(r \zeta)
$$

From now on, norm $\|\cdot\|$ and inner product $\langle\cdot, \cdot\rangle$ will be used for the space $H^{2}(\mathbb{T})$ and the reproducing kernel, i.e. the Szegö kernel $S(w, z)$ for $H^{2}(\mathbb{T})$ will be denoted by $K(w, z)$, to avoid any confusion:

$$
K(w, z)=K_{z}(w):=\frac{1}{1-w \bar{z}} \text { for } z, w \in \mathbb{D} .
$$

In order to see the connection between $H^{2}(\mathbb{T})$ and $H^{\infty}(\mathbb{D})$, let us consider any function $\psi \in H^{\infty}(\mathbb{D})$. For each $\psi$, there is a multiplication operator $M_{\psi}$ on $H^{2}(\mathbb{T})$ defined by

$$
\left(M_{\psi} h\right)(\zeta):=\psi(\zeta) h(\zeta) \text { for } \zeta \in \mathbb{T} \text { and } h \in H^{2}(\mathbb{T})
$$


or, with respect to analytic extension,

$$
\left(M_{\psi} h\right)(z)=\psi(z) h(z) \quad \text { for } \quad z \in \mathbb{D} \text {. }
$$

Lemma 4.1.3. Let $M_{\psi}$ be a multiplication operator on $H^{2}(\mathbb{T})$ defined as in (4.3). Then $\left\|M_{\psi}\right\|=\|\psi\|$ for all $\psi \in H^{\infty}(\mathbb{D})$.

Proof. Since

$$
\|\psi\|_{\infty}=\sup _{z \in \mathbb{D}}|\psi(z)|
$$

it is clear that $\left\|M_{\psi}\right\| \leq\|\psi\|_{\infty}$.

For the converse inequality, since

$$
K(z, z)=K_{z}(z)=\frac{1}{1-z \bar{z}}=\frac{1}{1-|z|^{2}}, \quad z \in \mathbb{D}
$$

then

$$
\begin{aligned}
\frac{|\psi(z)|}{1-|z|^{2}} & =|\psi(z)||K(z, z)|=\left|\psi(z) \|\left\langle K_{z}, K_{z}\right\rangle\right| \\
& =\left|\psi(z)\left\langle K_{z}, K_{z}\right\rangle\right|=\left|\left\langle\psi(z) K_{z}, K_{z}\right\rangle\right| \\
& =\left|\left\langle M_{\psi} K_{z}, K_{z}\right\rangle\right| \quad \text { since } \quad \psi K_{z}=M_{\psi} K_{z} \\
& \leq\left\|M_{\psi}\right\|\left\|K_{z}\right\|^{2} \\
& =\left\|M_{\psi}\right\|\left(\frac{1}{1-|z|^{2}}\right), \quad z \in \mathbb{D} .
\end{aligned}
$$

So, we get

$$
\frac{|\psi(z)|}{1-|z|^{2}} \leq\left\|M_{\psi}\right\| \frac{1}{1-|z|^{2}}
$$

which yields

$$
\sup _{z \in \mathbb{D}}|\psi(z)|=\|\psi\|_{\infty} \leq\left\|M_{\psi}\right\|
$$

Hence,

$$
\|\psi\|_{\infty}=\left\|M_{\psi}\right\| \quad \text { for } \quad \psi \in H^{\infty}(\mathbb{D}) .
$$

If $\psi, \phi \in H^{\infty}(\mathbb{D})$, we have $M_{\phi \cdot \psi}=M_{\phi} M_{\psi}$ for the map $\psi \longmapsto M_{\psi}$ from $H^{\infty}(\mathbb{D})$ to $B\left(H^{2}(\mathbb{T})\right)$ and $\psi$ is linear and multiplicative. 
Definition 4.1.4. The multiplication operator corresponding to the function $\phi(z):=z$, which is defined by

$$
S(\phi(z))=z \phi(z),
$$

is called the shift operator and is denoted by $S$.

It is easy to see that, for multiplication operator $M_{\psi}$ on $H^{2}(\mathbb{T})$ and shift operator $S$ on $B\left(H^{2}(\mathbb{T})\right)$, we have

$$
M_{\psi} S=S M_{\psi} .
$$

Theorem 4.1.5. If a continuous linear operator $T$ on $H^{2}(\mathbb{T})$ commutes with the shift operator $S$, i.e. $T S=S T$, then there exists uniquely $\psi \in H^{\infty}(\mathbb{D})$ for which $T=M_{\psi}$.

Proof. Let $l$ be the constant function with value 1 and let $\psi=T l$. Since

$$
K_{z}(w)=\frac{1}{1-w \bar{z}}=\sum_{n=0}^{\infty}(w \bar{z})^{n}, \quad z, w \in \mathbb{D},
$$

then

$$
K_{z}(z)=\sum_{n=0}^{\infty} \bar{z}^{n} z^{n}=\sum_{n=0}^{\infty} \bar{z}^{n} S^{n} l .
$$

Since by assumption $T$ is continuous on $H^{2}(\mathbb{T})$ and commutes with shift operator $S$, the above relation implies

$$
\begin{aligned}
T K_{z} & =\sum_{n=0}^{\infty} \bar{z}^{n} T S^{n} l=\sum_{n=0}^{\infty} \bar{z}^{n} S^{n} T l=\sum_{n=0}^{\infty} \bar{z}^{n} S^{n} \psi \quad \text { since } \psi=T l, \\
& =\psi \sum_{n=0}^{\infty} \bar{z}^{n} z^{n}=\psi K_{z}=M_{\psi} K_{z}, \quad z \in \mathbb{D} .
\end{aligned}
$$

So we get $T K_{z}=M_{\psi} K_{z}$ for $z \in \mathbb{D}$. Thus $\psi \in H^{\infty}(\mathbb{D})$. Moreover, since $T$ and $M_{\psi}$ are continuous and $\left\{K_{z}: z \in \mathbb{D}\right\}$ is total in $H^{2}(\mathbb{T})$, then we have $T=M_{\psi}$.

For the uniqueness part, suppose that there exists $\psi_{1}$ and $\psi_{2}$ such that $T=$ $M_{\psi_{1}}$ and $T=M_{\psi_{2}}$. It follows by (4.3) that

$$
T K_{z}=M_{\psi_{1}} K_{z}=\psi_{1} K_{z} \text { and } T K_{z}=M_{\psi_{2}} K_{z}=\psi_{2} K_{z}
$$


which yields

$$
\psi_{1} K_{z}=M_{\psi_{1}} K_{z}=M_{\psi_{2}} K_{z}=\psi_{2} K_{z}
$$

Thus $\psi_{1}=\psi_{2}$.

Corollary 4.1.6. Let $\mathcal{M}$ be a closed non-zero subspace of $H^{2}(\mathbb{T})$ and $S$ be the shift operator. If $\mathcal{M}$ is invariant for the adjoint $S^{*}$, i.e. $S^{*} h \in \mathcal{M}$ for all $h \in \mathcal{M}$, then the closed linear span of $\left\{S^{n}(\mathcal{M}): n=0,1, \ldots\right\}$ coincides with $H^{2}(\mathbb{T})$.

Proof. Let $\mathcal{M}$ be a closed non-zero subspace of $H^{2}(\mathbb{T})$. Denote by $\mathcal{C}$ the closed linear span of $\left\{S^{n}(\mathcal{M}): n=0,1, \ldots\right\}$. Since $S^{*} S=I$ and by assumption $S^{*}(\mathcal{M}) \subseteq \mathcal{M}$, then $\mathcal{C}$ is invariant for $S^{*}$, i.e. $S^{*}\left(S^{n}(g)\right) \subseteq \mathcal{C}$ for all $g \in \mathcal{M}$. In fact $S^{*}\left(S^{n}(g)\right)=S^{n-1}(g) \subseteq \mathcal{C}$ as $S S^{*}=I$. Clearly $\mathcal{C}$ is also invariant for $S$.

Let $P$ be the orthogonal projection to the closed linear $\operatorname{span} \mathcal{C}$.

Claim: $P S=S P$.

By using the definition of orthogonal projection, for $g=g_{1}+g_{2}$ where $g_{1} \in$ $\mathcal{M}$, and $g_{2} \in \mathcal{M}^{\perp}$, we have $P(g)=g_{1}$. Taking $h \in \mathcal{M},\left\langle h, S g_{2}\right\rangle=\left\langle S^{*} h, g_{2}\right\rangle=0$ since, by assumption, $S^{*} h \in \mathcal{M}$. In other words, $S g_{2} \in \mathcal{M}^{\perp}$. Then

$$
(P S)(g)=(P S)\left(g_{1}+g_{2}\right)=P\left(S\left(g_{1}\right)+S\left(g_{2}\right)\right)=S\left(g_{1}\right)=(S P)(g),
$$

that is, $P S=S P$ as claimed.

By previous theorem, $P=M_{\psi}$ for some $\psi \in H^{\infty}(\mathbb{D})$. Since $P^{2}=P$, we have $\left(M_{\psi}\right)^{2}=M_{\psi}$ which implies that $\psi(z)^{2}=\psi(z)$. But since $\psi \in H^{\infty}(\mathbb{D}), \quad \psi$ is analytic. This means, $\psi^{2}=\psi$ only if $\psi(z) \equiv 0$ or $\psi(z) \equiv 1$. By assumption since $\mathcal{M} \neq 0$, then $\psi(z) \equiv 1$. That is,

$$
M_{\psi} h=h \text { for all } h \in H^{2} .
$$

Hence the closed linear span of $\left\{S^{n}(\mathcal{M}): n=0,1, \ldots\right\}$ coincides with $H^{2}(\mathbb{T})$.

The following theorem states that two functions in $H^{2}(\mathbb{T})$ can coincide on a set of positive $\sigma$ - measure only when they represent one and the same element of $H^{2}(\mathbb{T})$. 
Theorem 4.1.7. A function $h \in H^{2}(\mathbb{T})$ can vanish on a measurable subset $\Lambda$ of $\mathbb{T}$ with positive $\sigma$-measure only if $h=0$.

Proof. Let $\Lambda$ be a measurable subset $\mathbb{T}$ with positive $\sigma$-measure. Consider the subspace $\mathcal{M}:=\left\{f \in H^{2}(\mathbb{T}): f(\zeta)=0\right.$ on $\Lambda \quad \sigma$-a.e (almost everywhere) $\}$.

Claim: $\mathcal{M}=\{0\}$.

$\mathcal{M}$ is a closed subspace since strong convergence in $H^{2}(\mathbb{T})$ implies convergence in measure on $\mathbb{T}$. Let $S$ be the shift operator. Since, by definition, $S(h) \in \mathcal{M}$ for all $h \in \mathcal{M}$, then $\mathcal{M}$ is invariant for $S$. Let $P$ be the orthogonal projection onto $\mathcal{M}$ and $l$ be the constant function with value 1 . Consider $g:=P l$. Then $l-g=l-P l=(I-P) l$ is orthogonal to $\mathcal{M}$. As $\mathcal{M}$ is invariant for $S$, it follows that $l-g$ is also orthogonal to $S^{n} g$ for $n=0,1, \ldots$

Since

$$
\left\langle S^{n} g, l\right\rangle=\left(S^{n} g\right)(0)=0 \text { for } n=1,2, \ldots,
$$

we have

$$
\begin{aligned}
0 & =\left\langle S^{n} g, l-g\right\rangle=\int_{\mathbb{T}}\left(S^{n} g\right)(\zeta) \overline{(l-g)(\zeta)} d \sigma(\zeta) \\
& =\int_{\mathbb{T}} S^{n}(\zeta) g(\zeta) \overline{l(\zeta)} d \sigma(\zeta)-\int_{\mathbb{T}}\left(S^{n} g\right)(\zeta) \overline{(g)(\zeta)} d \sigma(\zeta) \\
& =-\int_{\mathbb{T}} \zeta^{n}|g(\zeta)|^{2} d \sigma(\zeta) \quad \text { as } \quad\left\langle S^{n} g, l\right\rangle=0 .
\end{aligned}
$$

This means that all Fourier coefficients of $|g|^{2}$, except the constant term, vanish, thus $|g(\zeta)|^{2}$ is constant on $\mathbb{T} \quad \sigma-$ a.e. Since $g=P l \in \mathcal{M}, g(\zeta)$ vanishes on $\Lambda$ of positive $\sigma$-measure which implies that $g=0$, i.e. $g=P l=0$. This means $l$ is orthogonal to $\mathcal{M}$. Then for each $f \in \mathcal{M}$, as $S f \in \mathcal{M}$, we have $\left(S^{*} f\right)(\zeta)=\bar{\zeta} f(\zeta)$, hence $S^{*} f$ vanishes on $\Lambda \sigma-a . e$, which means that $\mathcal{M}$ is invariant for $S^{*}$. Hence, since $\mathcal{M}$ is invariant for both $S$ and $S^{*}$ and $l$ is not in $\mathcal{M}$, by previous corollary, $\mathcal{M}=\{0\}$.

Corollary 4.1.8. Let $P_{+}$be the orthogonal projection from the Hilbert space $L^{2}(\mathbb{T})$ to $H^{2}(\mathbb{T})$. If $\Lambda$ is a measurable subset of $\mathbb{T}$ with positive $\sigma$-measure, then $\left\{P_{+}\left(\chi_{\Lambda} f\right): f \in L^{2}(\mathbb{T})\right\}$ is a dense subspace of $H^{2}(\mathbb{T})$, where $\chi_{\Lambda}(\zeta)=1$ is the characteristic function of $\Lambda$. 
Proof. We show that, if $H^{2}(\mathbb{T}) \ni h \perp \chi_{\Lambda} f$ for all $f \in L^{2}(\mathbb{T})$, then $h=0$. To see this, consider the previous theorem,

$$
\begin{aligned}
0 & =\left\langle\chi_{\Lambda} h, h\right\rangle=\int_{\Lambda}\left(\chi_{\Lambda} h\right)(\zeta) \bar{h}(\zeta) d \sigma(\zeta) \\
& =\int_{\Lambda} h(\zeta) \bar{h}(\zeta) d \sigma(\zeta)=\int_{\Lambda}|h(\zeta)|^{2} d \sigma(\zeta),
\end{aligned}
$$

implies that $h$ vanishes on $\Lambda \quad \sigma$-a.e. Hence $\left\{P_{+}\left(\chi_{\Lambda} f\right): f \in L^{2}(\mathbb{T})\right\}$ is a dense subspace of $H^{2}(\mathbb{T})$.

A consequence of the above proof is that, $\left\{P_{+}\left(\chi_{\Lambda} g\right): g \in H^{2}(\mathbb{T})\right\}$ is dense in $\left.H^{2}(\mathbb{T})\right\}$.

\subsection{Interpolation Inside Unit Disc}

In this section we consider interpolation on finite and infinite subsets of the unit disc. In the following theorem we consider interpolation on a finite subset.

Theorem 4.2.1. (Nevanlinna-Pick) Let $X=\left\{z_{1}, \ldots, z_{n}\right\}$ be a finite subset of $\mathbb{D}$ and $\phi$ be a function on $X$. Then, in order for there to exist an analytic function $\psi \in H^{\infty}(\mathbb{D})$ such that

$$
\|\psi\|_{\infty} \leq 1 \quad \text { and } \quad \psi\left(z_{i}\right)=\phi\left(z_{i}\right), \quad(i=1,2, \ldots, n)
$$

it is necessary and sufficient that the kernel $\left[\frac{1-\phi\left(z_{i}\right) \overline{\phi\left(z_{j}\right)}}{1-z_{i} \bar{z}_{j}}\right]$ on $X$ is positive definite, that is,

$$
\sum_{i, j=1}^{n} \bar{\xi}_{i} \xi_{j} \frac{1-\phi\left(z_{i}\right) \overline{\phi\left(z_{j}\right)}}{1-z_{i} \bar{z}_{j}} \geq 0 \text { for all }\left\{\xi_{i}\right\}_{i=1}^{n} \subset \mathbb{C}
$$

Proof. Necessity: Suppose that there exists $\psi \in H^{\infty}(\mathbb{D})$ satisfying (4.5).

Claim: $M_{\psi}^{*} K_{z}=\overline{\psi(z)} K_{z} \quad$ where $\quad K_{z}(w)=K(w, z)=\frac{1}{1-w \bar{z}} \quad$ for $\quad w, z \in \mathbb{D}$.

By using the properties of multiplication operators and reproducing kernels, 
we get

$$
\begin{aligned}
\left(M_{\psi}^{*} K_{z}\right)(w) & =\left\langle M_{\psi}^{*} K_{z}, K_{w}\right\rangle=\left\langle K_{z}, M_{\psi} K_{w}\right\rangle=\left\langle K_{z}, \psi(z) K_{w}\right\rangle \\
& =\overline{\psi(z)}\left\langle K_{z}, K_{w}\right\rangle=\overline{\psi(z)} K(w, z)=\overline{\psi(z)} \overline{K(z, w)} \\
& =\overline{\psi(z)} K_{z}(w) .
\end{aligned}
$$

Hence $M_{\psi}^{*} K_{z}=\overline{\psi(z)} K_{z}$ for $z \in \mathbb{D}$, as claimed.

Since, by assumption, $\|\psi\|_{\infty} \leq 1$, and by Lemma 4.1 .3 we have $\left\|M_{\psi}\right\|=$ $\|\psi\|_{\infty}$, combining these we get $\left\|M_{\psi}\right\|=\left\|M_{\psi}^{*}\right\| \leq 1$. Then using Claim and assumption,

$$
\begin{aligned}
0 & \leq\left\|\sum_{j=1}^{n} \xi_{j} K_{z_{j}}\right\|^{2}-\left\|M_{\psi}^{*}\left(\sum_{j=1}^{n} \xi_{j} K_{z_{j}}\right)\right\|^{2} \quad \text { as } \quad\left\|M_{\psi}^{*}\right\| \leq 1 \\
& =\left\|\sum_{j=1}^{n} \xi_{j} K_{z_{j}}\right\|^{2}-\left\|\sum_{j=1}^{n} \xi_{j} M_{\psi}^{*} K_{z_{j}}\right\|^{2} \\
& =\left\|\sum_{j=1}^{n} \xi_{j} K_{z_{j}}\right\|^{2}-\left\|\sum_{j=1}^{n} \xi_{j} \overline{\psi\left(z_{j}\right)} K_{z_{j}}\right\|^{2} \quad \text { as } \quad M_{\psi}^{*} K_{z}=\overline{\phi(z)} K_{z} \\
& =\left\langle\sum_{j=1}^{n} \xi_{j} K_{z_{j}}, \sum_{j=1}^{n} \xi_{j} K_{z_{j}}\right\rangle-\left\langle\sum_{j=1}^{n} \xi_{j} \overline{\psi\left(z_{j}\right)} K_{z_{j}}, \sum_{j=1}^{n} \xi_{j} \overline{\psi\left(z_{j}\right)} K_{z_{j}}\right\rangle \\
& =\sum_{i, j=1} \overline{\xi_{i}} \xi_{j}\left(1-\psi\left(z_{i}\right) \overline{\psi\left(z_{j}\right)}\right)\left\langle K_{z_{j}}, K_{z_{i}}\right\rangle \\
& =\sum_{i, j=1} \overline{\xi_{i}} \xi_{j} \frac{1-\phi\left(z_{i}\right) \overline{\phi\left(z_{j}\right)}}{1-z_{i} \bar{z}_{j}} \quad \text { for all } \quad\left\{\xi_{i}\right\}_{i=1}^{n} \subset \mathbb{C},
\end{aligned}
$$

which is the desired result.

Sufficiency: Suppose that the kernel $\left[\frac{1-\phi\left(z_{i}\right) \overline{\phi\left(z_{j}\right)}}{1-z_{i} \bar{z}_{j}}\right]$ on $X$ is positive definite, that is,

$$
\left.\sum_{i, j=1}^{n} \bar{\xi}_{i} \xi_{j} \frac{1-\phi\left(z_{i}\right) \overline{\phi\left(z_{j}\right)}}{1-z_{i} \bar{z}_{j}} \geq 0 \text { for all [ } \xi_{i}\right] .
$$

Denote by $\mathcal{M}$ the closed linear span of $K_{z_{j}},(j=1,2, \ldots, n)$, and $S$ shift operator. By the Claim in the necessity part, we have $S^{*} K_{z}=\bar{z} K_{z}$ for any $z \in \mathbb{D}$. This implies that $\mathcal{M}$ is invariant for $S^{*}$. Then consider the following function

$$
\tilde{\psi}(z):=\sum_{i=1}^{n} \frac{\prod_{j \neq i} \frac{z-z_{j}}{1-z \overline{z_{j}}}}{\prod_{j \neq i} \frac{z_{i}-z_{j}}{1-z_{i} \overline{z_{j}}}} \phi\left(z_{i}\right) .
$$


Note that this function $\tilde{\psi} \in H^{\infty}(\mathbb{D})$ satisfies the following

$$
\tilde{\psi}\left(z_{i}\right)=\phi\left(z_{i}\right) \quad(i=1,2, \ldots, n) .
$$

Let $P$ be the orthogonal projection onto $\mathcal{M}$. Now define the linear operator $T$ on $\mathcal{M}$ by

$$
T=\left.P M_{\tilde{\psi}}\right|_{\mathcal{M}} .
$$

Since (4.7) is satisfied, then (4.6) becomes

$$
\sum_{i, j=1}^{n} \bar{\xi}_{i} \xi_{j} \frac{1-\tilde{\psi}\left(z_{i}\right) \overline{\tilde{\psi}\left(z_{j}\right)}}{1-z_{i} \bar{z}_{j}} \geq 0 \quad \text { for } \quad\left\{\xi_{i}\right\}_{i=1}^{n} \subset \mathbb{C} .
$$

It follows that $\left\|T^{*} h\right\| \leq\|h\|$ for $h \in \mathcal{M}$ and hence $\|T\| \leq 1$. To be able to finish this proof we need the following lemma.

Lemma 4.2.2. Suppose that $\mathcal{M}_{0}$ is a non-zero closed subspace of $H^{2}(\mathbb{T})$, invariant for the adjoint $S^{*}$, and that $T_{0}$ is a continuous linear operator on $\mathcal{M}_{0}$, commuting with the restricted shift $S_{0}:=\left.P_{0} S\right|_{\mathcal{M}_{0}}$ where $P_{0}$ is the orthogonal projection onto $\mathcal{M}_{0}$, that is,

$$
T_{0} S_{0}=S_{0} T_{0} .
$$

Then the closed linear span $\mathcal{M}_{1}$ of $\mathcal{M}_{0}$ and $S \mathcal{M}_{0}$ is invariant for $S^{*}$ and there is a continuous linear operator $T_{1}$ on $\mathcal{M}_{1}$ such that

$$
P_{0} T_{1}=T_{0} P_{0}, \quad\left\|T_{1}\right\|=\left\|T_{0}\right\|, \quad \text { and } \quad T_{1} S_{1}=S_{1} T_{1},
$$

where $S_{1}=\left.P_{1} S\right|_{\mathcal{M}_{1}}, P_{1}$ being the orthogonal projection onto $\mathcal{M}_{1}$.

Proof. We prove this lemma in five steps.

Step 1: $\mathcal{M}_{1}$ is invariant for $S^{*}$.

By definition, $\mathcal{M}_{0}$ is invariant for $S^{*}$ means $S^{*}\left(\mathcal{M}_{0}\right) \subseteq \mathcal{M}_{0}$. Since $S^{*} S=I$, we have $S^{*}\left(S\left(\mathcal{M}_{0}\right)\right)=\mathcal{M}_{0}$. Then, since $\mathcal{M}_{1}$ is defined as closed linear span of $\mathcal{M}_{0}$ and $S\left(\mathcal{M}_{0}\right)$, we have $\mathcal{M}_{1}$ is invariant for $S^{*}$.

Step 2: $S_{1}=S P_{0}$ on $\mathcal{M}_{1}$. 
Let $\mathcal{M}_{1} \ni g$ be orthogonal on $\mathcal{M}_{0}$. Then, since by Step $1, S^{*} h \in \mathcal{M}_{1}$ for $h \in \mathcal{M}_{0}$, it follows that

$$
\langle S g, h\rangle=\left\langle g, S^{*} h\right\rangle=0 \text { for } h \in \mathcal{M}_{0},
$$

that is, $S g$ is orthogonal to $\mathcal{M}_{0}$ and

$$
\langle S g, S h\rangle=\langle g, h\rangle=0 \quad \text { for } g \in \mathcal{M}_{1}, h \in \mathcal{M}_{0} .
$$

This implies that $S g$ is also orthogonal to $S\left(\mathcal{M}_{0}\right)$. Now, since $S_{1}=P_{1} S$ on $\mathcal{M}_{1}$, where $P_{1}$ is the orthogonal projection onto $\mathcal{M}_{1}$, then we get

$$
S_{1} g=P_{1} S g=P_{1}(S(g))=0 \text { because } S(g) \perp \mathcal{M}_{0} .
$$

It follows that $S_{1}=S_{1} P_{0}$ on $\mathcal{M}_{1}$. Since $S\left(\mathcal{M}_{0}\right) \subseteq \mathcal{M}_{1}$, we obtain

$$
S_{1}=S_{1} P_{0}=S P_{0}
$$

Before the third step, without loss of generality we assume that we have $\left\|T_{0}\right\|=1$.

Step 3: $\left\|\left(S_{1}-S_{0} P_{0}\right) T_{0} P_{0} h\right\| \leq\left\|\left(I_{1}-\left(T_{0} P_{0}\right)^{*}\left(T_{0} P_{0}\right)\right)^{1 / 2} \cdot S_{1} h\right\| \quad$ for $h \in \mathcal{M}_{1}$, and $I_{1}$ is the identity operator of $\mathcal{M}_{1}$.

By assumptions and previous steps, we have the following,

$$
\begin{aligned}
\left\|\left(S_{1}-S_{0} P_{0}\right) T_{0} P_{0} h\right\|^{2} & =\left\|\left(S P_{0}-S_{0} P_{0}\right) T_{0} P_{0} h\right\|^{2}=\left\|\left(S-S_{0}\right) P_{0} T_{0} P_{0} h\right\|^{2} \\
& =\left\|\left(S-S P_{0}\right) P_{0} T_{0} P_{0} h\right\|^{2}=\left\|\left(I-P_{0}\right) S P_{0} T_{0} P_{0} h\right\|^{2} \\
& =\left\|S P_{0} T_{0} P_{0} h\right\|^{2}-\left\|S_{0} T_{0} P_{0} h\right\|^{2} \\
& \leq\left\|P_{0} h\right\|^{2}-\left\|S_{0} T_{0} P_{0} h\right\|^{2}=\left\|S P_{0} h\right\|^{2}-\left\|T_{0} S_{0} P_{0} h\right\|^{2} \\
& =\left\|S_{1} h\right\|^{2}-\left\|T_{0} P_{0} S_{1} h\right\|^{2} \\
& =\left\langle S_{1} h, S_{1} h\right\rangle-\left\langle T_{0} P_{0} S_{1} h, T_{0} P_{0} S_{1} h\right\rangle \\
& =\left\langle S_{1} h, S_{1} h\right\rangle-\left\langle\left(T_{0} P_{0}\right)^{*}\left(T_{0} P_{0}\right) S_{1} h, S_{1} h\right\rangle \\
& =\left\langle S_{1} h-\left(T_{0} P_{0}\right)^{*}\left(T_{0} P_{0}\right), S_{1} h\right\rangle \\
& =\left\langle\left(I_{1}-\left(T_{0} P_{0}\right)^{*}\left(T_{0} P_{0}\right)\right) S_{1} h, S_{1} h\right\rangle .
\end{aligned}
$$


Considering the identity operator $I_{1}$ of $\mathcal{M}_{1}$, since $\left\|T_{0} P_{0}\right\| \leq 0$ implies $I_{1}-\left(T_{0} P_{0}\right)^{*}\left(T_{0} P_{0}\right) \geq 1$, we can consider the positive definite square root

$$
\left(I_{1}-\left(T_{0} P_{0}\right)^{*}\left(T_{0} P_{0}\right)\right)^{1 / 2} .
$$

So from the above inequality it follows as

$$
\left\|\left(S_{1}-S_{0} P_{0}\right) T_{0} P_{0} h\right\| \leq\left\|\left(I_{1}-\left(T_{0} P_{0}\right)^{*}\left(T_{0} P_{0}\right)\right)^{1 / 2} \cdot S_{1} h\right\| \quad \text { for } h \in \mathcal{M}_{1} .
$$

This means that we have a well-defined linear operator from the range of $\left(I_{1}-\left(T_{0} P_{0}\right)^{*}\left(T_{0} P_{0}\right)\right)^{1 / 2} \cdot S_{1}$ to the range of $\left(S_{1}-S_{0} P_{0}\right) T_{0} P_{0}$ and of norm $\leq 1$. Now consider its continuous extension, composed with the orthogonal projection to the closure of the range of $\left(I_{1}-\left(T_{0} P_{0}\right)^{*}\left(T_{0} P_{0}\right)\right)^{1 / 2} \cdot S_{1}$, then we can conclude that there is a continuous linear operator $L$ from $\mathcal{M}_{1}$ to the closure of the range of $S_{1}-S_{0} P_{0}$ such that $\|L\| \leq 1$ and

$$
\left(S_{1}-S_{0} P_{0}\right) T_{0} P_{0}=L\left(I_{1}-\left(T_{0} P_{0}\right)^{*}\left(T_{0} P_{0}\right)\right)^{1 / 2} \cdot S_{1} .
$$

Define $T_{1}$ by

$$
T_{1}=T_{0} P_{0}+L\left(I_{1}-\left(T_{0} P_{0}\right)^{*}\left(T_{0} P_{0}\right)\right)^{1 / 2} .
$$

Step 4: $\quad P_{0} T_{1}=T_{0} P_{0}$ and $T_{1} S_{1}=S_{1} T_{1}$.

Since the range of $L$ is contained in the closure of $S_{1}-S_{0} P_{0}$, applying $P_{0}$ to both sides of (4.12), we get

$$
\begin{aligned}
P_{0} T_{1} & =P_{0} T_{0} P_{0}+P_{0} L\left(I_{1}-\left(T_{0} P_{0}\right)^{*}\left(T_{0} P_{0}\right)\right)^{1 / 2} \\
& =P_{0} T_{0} P_{0}+P_{0}\left(S_{1}-S_{0} P_{0}\right) T_{0} P_{0} \\
& =P_{0} T_{0} P_{0} \quad \text { since } \quad P_{0}\left(S_{1}-S_{0} P_{0}\right)=0 .
\end{aligned}
$$

Similarly,

$$
\begin{aligned}
T_{1} S_{1} & =T_{0} P_{0} S_{1}+L\left(I_{1}-\left(T_{0} P_{0}\right)^{*}\left(T_{0} P_{0}\right)\right)^{1 / 2} S_{1} \\
& =T_{0} S_{0} P_{0}+\left(S_{1}-S_{0} P_{0}\right) T_{0} P_{0} \\
& =S_{1} T_{0} P_{0} \\
& =S_{1} T_{1} .
\end{aligned}
$$


Hence

$$
P_{0} T_{1}=T_{0} P_{0} \quad \text { and } \quad T_{1} S_{1}=S_{1} T_{1} .
$$

Step 5: $\left\|T_{1}\right\|=\left\|T_{0}\right\|$.

Since $P_{0} L=0$, we have

$$
\begin{aligned}
\left\|T_{1} h\right\|^{2} & =\left\|T_{0} P_{0} h\right\|^{2}+\left\|L\left(I_{1}-\left(T_{0} P_{0}\right)^{*}\left(T_{0} P_{0}\right)\right)^{1 / 2} h\right\|^{2} \\
& \leq\left\|T_{0} P_{0} h\right\|^{2}+\|h\|^{2}-\left\|T_{0} P_{0} h\right\|^{2} \quad \text { by } \quad\|L\| \leq 1 \\
& =\|h\|^{2},
\end{aligned}
$$

which yields $\left\|T_{1}\right\| \leq 1=\left\|T_{0}\right\|$.

Conversely,

$$
\left\|T_{0}\right\|=\left\|T_{0} P_{0}\right\|=\left\|P_{0} T_{1}\right\| \leq\left\|T_{1}\right\| .
$$

So we have $\left\|T_{1}\right\|=\left\|T_{0}\right\|$.

Therefore $T_{1}$ satisfies all the requirements in (4.10) of Lemma.

Let us return to the proof of sufficiency part of previous theorem. To avoid confusion, let us take $\mathcal{M}_{0}:=\mathcal{M}$ and $T_{0}:=T$ in Lemma. We have that $\mathcal{M}_{0}$ is invariant under $S^{*}$ and $\left\|T_{0}\right\| \leq 1$. Since $T_{0}=\left.P_{0} M_{\tilde{\psi}}\right|_{\mathcal{M}_{0}}$ and $S_{0}=\left.P_{0} S\right|_{\mathcal{M}_{0}}$ and $\mathcal{M}_{0}{ }^{\perp}$ is invariant under $S$ and $M_{\tilde{\psi}}$, we have $P_{0} M_{\tilde{\psi}} P_{0}=P_{0} M_{\tilde{\psi}}$ and $P_{0} S P_{0}=P_{0} S$ such that

$$
T_{0} S_{0}=\left.P_{0} M_{\tilde{\psi}} P_{0} S\right|_{\mathcal{M}_{0}}=\left.P_{0} M_{\tilde{\psi}} S\right|_{\mathcal{M}_{0}}=\left.P_{0} S M_{\tilde{\psi}}\right|_{\mathcal{M}_{0}}=S_{0} T_{0}
$$

Now we can change our theorem into the lemma in such a way that, we have to find a sequence of linear operators $T_{n}$ on $\mathcal{M}_{n}$, the closed linear span of $\mathcal{M}_{n-1}$ and $S\left(\mathcal{M}_{n-1}\right), \quad(n=1,2, \ldots)$, such that

$$
P_{n-1} T_{n}=T_{n-1} P_{n-1}, \quad\left\|T_{n}\right\|=\left\|T_{n-1}\right\| \text { and } T_{n} S_{n}=S_{n} T_{n},
$$

where, for each $n, P_{n}$ is the orthogonal projection onto $\mathcal{M}_{n}$ and $S_{n}=\left.P_{n} S\right|_{\mathcal{M}_{n}}$. By Corollary 4.1.6, the closed linear span of $\left\{\mathcal{M}_{n}: n=0,1, \ldots\right\}$ which is equal to that of $\left\{S^{n}\left(\mathcal{M}_{0}\right): n=0,1, \ldots\right\}$ must coincide with $H^{2}(\mathbb{T})$. This means that, $P_{n}$ strongly converges to $I$ as $n \rightarrow \infty$. By the equation (4.13), we reach

$$
\left\|T_{n} P_{n}\right\| \leq 1 \text { and } P_{n} T_{m} P_{m}=T_{n} P_{n} \text { for } n \leq m,
$$


and hence it follows that there is a continuous linear operator $T_{\infty}$ on $\mathcal{H}^{2}(\mathbb{T})$ such that

$$
\left\|T_{\infty}\right\| \leq 1 \quad \text { and } \quad P_{n} T_{\infty}=T_{n} P_{n} \quad \text { for } \quad n=0,1, \ldots
$$

Claim: $T_{\infty} S=S T_{\infty}$

Since $P_{n}$ strongly converges to $I$ as $n \rightarrow \infty$, then $S_{n} P_{n}$ strongly converges to $S$. By (4.15), we have $T_{\infty}=\lim _{n \rightarrow \infty} P_{n} T_{n} P_{n}$. These imply

$$
\begin{aligned}
S T_{\infty} & =S \lim _{n \rightarrow \infty} P_{n} T_{n} P_{n}=\lim _{n \rightarrow \infty} S_{n} T_{n} P_{n} \\
& =\lim _{n \rightarrow \infty} T_{n} S_{n} P_{n} \quad \text { by } \quad(4.13) \\
& =T_{\infty}\left(\lim _{n \rightarrow \infty} S_{n} P_{n}\right)=T_{\infty} S .
\end{aligned}
$$

Then by Theorem 4.1.5, there exists $\psi \in H^{\infty}(\mathbb{D})$ such that

$$
\|\psi\|_{\infty} \leq 1 \text { and } M_{\psi}=T_{\infty} .
$$

Then by (4.15) and (4.16) we reach to

$$
\left.P_{0} M_{\psi}\right|_{\mathcal{M}_{0}}=\left.P_{0} T_{\infty}\right|_{\mathcal{M}_{0}}=T_{0} .
$$

Finally, by (4.7) and (4.16),

$$
\begin{aligned}
\frac{\phi\left(z_{i}\right)}{1-\left|z_{i}\right|^{2}} & =\phi\left(z_{i}\right)\left\langle K_{z_{i}}, K_{z_{i}}\right\rangle=\left\langle T_{0} K_{z_{i}}, K_{z_{i}}\right\rangle \\
& =\left\langle T_{\infty} K_{z_{i}}, K_{z_{i}}\right\rangle=\left\langle M_{\psi} K_{z_{i}}, K_{z_{i}}\right\rangle \\
& =\frac{\psi\left(z_{i}\right)}{1-\left|z_{i}\right|^{2}} .
\end{aligned}
$$

Hence $\psi$ satisfies all the requirements in (4.5) of Theorem 4.2.1.

In the following corollary we consider interpolation on an infinite subset of the unit disc.

Corollary 4.2.3. Let $X$ be an infinite subset of $\mathbb{D}$, and $\phi$ a function on $X$. In order for there to exist a function $\psi \in H^{\infty}(\mathbb{D})$ such that

$$
\|\psi\|_{\infty} \leq 1 \quad \text { and } \psi(z)=\phi(z) \quad \text { for } \quad z \in X,
$$

it is necessary and sufficient that the kernel $\left[\frac{1-\phi\left(z_{i}\right) \overline{\phi\left(z_{j}\right)}}{1-z_{i} \bar{z}_{j}}\right]$ on $X$ is positive definite. 
Proof. This follows by an application of Zorn's Lemma and the previous theorem.

\subsection{Interpolation on the Boundary}

We will end this chapter by a theorem of interpolation on the boundary of unit disk $\mathbb{D}$. Before this we need the following lemma.

Lemma 4.3.1. Let $\Lambda$ be a measurable subset of $\mathbb{T}$ and $\phi$ a bounded measurable function on $\Lambda$. Then in order for there to exist $\psi \in H^{\infty}(\mathbb{D})$ such that

$$
\|\psi\|_{\infty} \leq 1 \quad \text { and } \psi(\zeta)=\phi(\zeta) \text { for } \zeta \in \Lambda \text { } \sigma-\text { a.e. },
$$

it is necessary and sufficient that

$$
\left\|P_{+}\left(\bar{\phi} \chi_{\Lambda} f\right)\right\| \leq\left\|P_{+}\left(\chi_{\Lambda} f\right)\right\| \text { for } f \in L^{2}(\mathbb{T}) .
$$

Proof. Suppose that (4.18) is satisfied for $\psi \in H^{\infty}(\mathbb{D})$. Then

$$
\begin{aligned}
\left\|P_{+}\left(\bar{\phi} \chi_{\Lambda} f\right)\right\| & =\sup _{g \in H^{2}(\mathbb{D}),\|g\| \leq 1}\left|\left\langle\bar{\phi} \chi_{\Lambda} f, g\right\rangle\right|=\sup _{g \in H^{2}(\mathbb{D}),\|g\| \leq 1}\left|\left\langle\chi_{\Lambda} f, \psi g\right\rangle\right| \\
& \leq\|\psi\|_{\infty}\left\|P_{+}\left(\chi_{\Lambda} f\right)\right\| \\
& \leq\left\|P_{+}\left(\chi_{\Lambda} f\right)\right\| \quad \text { since }\|\psi\|_{\infty} \leq 1 .
\end{aligned}
$$

Hence (4.19) is satisfied.

Conversely, suppose that (4.19) is satisfied. Since by Corollary 4.1.8 $\left\{P_{+}\left(\chi_{\Lambda} f\right): f \in L^{2}(\mathbb{T})\right\}$ is a dense subspace of $H^{2}(\mathbb{T})$, then there exists an operator $T$ such that

$$
\|T\| \leq 1 \quad \text { and } T P_{+}\left(\chi_{\Lambda} f\right)=P_{+}\left(\bar{\phi} \chi_{\Lambda} f\right) \quad \text { for } f \in L^{2}(\mathbb{T}) .
$$

Claim: $T S^{*}=S^{*} T$. 
Denote by $\tilde{S}$ the multiplication by the function $k(\zeta):=\zeta$ in $L^{2}(\mathbb{T})$. Then we have $S^{*} P_{+}=P_{+} S^{*}$. It follows that

$$
\begin{aligned}
S^{*} T P_{+}\left(\chi_{\Lambda} f\right) & =S^{*} P_{+}\left(\bar{\phi} \chi_{\Lambda} f\right)=P_{+} \tilde{S}^{*}\left(\bar{\phi} \chi_{\Lambda} f\right) \\
& =P_{+}\left(\bar{\phi} \chi_{\Lambda} \tilde{S}^{*} f\right)=T P_{+}\left(\chi_{\Lambda} \tilde{S}^{*} f\right) \\
& =T P_{+}\left(\tilde{S}^{*}\left(\chi_{\Lambda} f\right)\right)=T S^{*} P_{+}\left(\chi_{\Lambda} f\right) .
\end{aligned}
$$

Since $\left\{P_{+}\left(\chi_{\Lambda} f\right): f \in L^{2}(\mathbb{T})\right\}$ is dense in $H^{2}(\mathbb{T})$, the above equation implies

$$
S^{*} T=T S^{*}
$$

By Theorem 4.1 .5 there exists $\psi \in H^{\infty}(\mathbb{D})$ such that

$$
\|\psi\|_{\infty}=\left\|T^{*}\right\| \leq 1 \text { and } T^{*}=M_{\psi} .
$$

Finally, it remains to show $\psi(\zeta)=\phi(\zeta)$ for $\zeta \in \Lambda \sigma-$ a.e. Take $f \in L^{2}(\mathbb{T})$. Then

$$
\begin{aligned}
\int_{\Lambda} \psi(\zeta) \overline{f(\zeta)} d \sigma(\zeta) & =\left\langle T^{*} l, \chi_{\Lambda} f\right\rangle=\left\langle T^{*} l, P_{+}\left(\chi_{\Lambda} f\right)\right\rangle \\
& =\left\langle l, T P_{+}\left(\chi_{\Lambda} f\right)\right\rangle=\left\langle l, P_{+}\left(\bar{\phi} \chi_{\Lambda} f\right)\right\rangle \\
& =\left\langle l, \bar{\phi} \chi_{\Lambda} f\right\rangle=\int_{\Lambda} \phi(\zeta) \tilde{f}(\zeta) d \sigma(\eta)
\end{aligned}
$$

which yields that

$$
\psi(\zeta)=\phi(\zeta) \quad \text { for } \zeta \in \Lambda, \sigma-\text { a.e. }
$$

Theorem 4.3.2. Let $\Lambda$ be a measurable subset of $\mathbb{T}$ with positive $\sigma$-measure and $\phi$ a bounded measurable function on $\Lambda$. Then in order for there to exist $\psi \in H^{\infty}(\mathbb{D})$ such that

$$
\|\psi\|_{\infty} \leq 1 \text { and } \psi(\zeta)=\phi(\zeta) \text { for } \zeta \in \Lambda \sigma-\text { a.e. }
$$

it is necessary and sufficient that

$$
\lim _{r \rightarrow 1} \int_{\Lambda} \int_{\Lambda}\left(\frac{1-\phi(\xi) \overline{\phi(\zeta)}}{1-r^{2} \xi \bar{\zeta}}\right) \overline{f(\xi)} f(\zeta) d \sigma(\xi) d \sigma(\zeta) \geq 0 \text { for all } f \in L^{2}(\mathbb{T}) .
$$

Proof. Recall that in Chapter 2, the orthogonal projection $P_{H^{2}}$ was expressed in terms of the Szegö kernel;

$$
\left(P_{H^{2}} g\right)(z)=\left\langle g, K_{z}\right\rangle_{L^{2}}, \quad \text { for } z \in \mathbb{D} \text { and } g \in L^{2}(\mathbb{T}),
$$


and

$$
\left(P_{H^{2}} g\right)(\zeta)=\lim _{r \rightarrow 1}\left\langle g, K_{r \zeta}\right\rangle \text { for almost all } \zeta \in \mathbb{T} .
$$

Then for $0<r<1$ and $\eta \in \mathbb{T}$, by above equations we get the followings;

$$
\left(P_{+} g\right)(r \eta)=\int_{\mathbb{T}} g(\zeta) \frac{1}{1-r \eta \bar{\zeta}} d \sigma(\zeta)
$$

and

$$
\begin{aligned}
\lim _{r \rightarrow 1} & \int_{\mathbb{T}}\left|\left(P_{+} g\right)(r \eta)-\left(P_{+} g\right)(\eta)\right|^{2} d \sigma(\eta) \\
& =\lim _{r \rightarrow 1} \int_{\mathbb{T}}\left|\int_{\mathbb{T}} g(\zeta)\left(\frac{1}{1-r \eta \bar{\zeta}}\right) d \sigma(\zeta)-\int_{\mathbb{T}} g(\zeta)\left(\frac{1}{1-\eta \bar{\zeta}}\right) d \sigma(\zeta)\right|^{2} d \sigma(\eta) \\
& =\lim _{r \rightarrow 1} \int_{\mathbb{T}}\left|\int_{\mathbb{T}} g(\zeta)\left(\frac{1}{1-r \eta \bar{\zeta}}-\frac{1}{1-\eta \bar{\zeta}}\right) d \sigma(\zeta)\right|^{2} d \sigma(\eta) \\
& =\lim _{r \rightarrow 1} \int_{\mathbb{T}}\left|\int_{\mathbb{T}} g(\zeta)\left(\frac{r \eta \bar{\zeta}-\eta \bar{\zeta}}{1-\eta \bar{\zeta}-r \eta \bar{\zeta}+r \eta|\bar{\zeta}|^{2}}\right) d \sigma(\zeta)\right|^{2} d \sigma(\eta) \\
& =0 .
\end{aligned}
$$

It follows that

$$
\begin{aligned}
& \left\|P_{+} g\right\|^{2}=\left\langle P_{+} g, P_{+} g\right\rangle=\lim _{r \rightarrow 1} \int_{\mathbb{T}}\left|\left(P_{+} g\right)(r \eta)\right|^{2} d \sigma(\eta) \\
& =\lim _{r \rightarrow 1} \int_{\mathbb{T}}\left(\int_{\mathbb{T}} \int_{\mathbb{T}} \frac{g(\zeta)}{1-r \eta \bar{\zeta}} \frac{\overline{g(\varepsilon)}}{1-r \bar{\eta} \varepsilon} d \sigma(\varepsilon) d \sigma(\zeta)\right) d \sigma(\eta) \\
& =\lim _{r \rightarrow 1} \int_{\mathbb{T}} \int_{\mathbb{T}}\left(\int_{\mathbb{T}} \frac{1}{1-r \eta \bar{\zeta}} \frac{1}{1-r \bar{\eta} \varepsilon} d \sigma(\eta)\right) \overline{g(\varepsilon)} g(\zeta) d \sigma(\varepsilon) d \sigma(\eta) \\
& =\lim _{r \rightarrow 1} \int_{\mathbb{T}} \int_{\mathbb{T}}\left\langle K_{r \eta}, K_{r \varepsilon}\right\rangle \overline{g(\varepsilon)} g(\zeta) d \sigma(\varepsilon) d \sigma(\eta) \\
& =\lim _{r \rightarrow 1} \int_{\mathbb{T}} \int_{\mathbb{T}} \frac{1}{1-r^{2} \varepsilon \bar{\zeta}} \overline{g(\varepsilon)} g(\zeta) d \sigma(\varepsilon) d \sigma(\eta) .
\end{aligned}
$$

Therefore for all $g \in \mathcal{L}^{2}(\mathbb{T})$, we have

$$
\left\|P_{+} g\right\|^{2}=\lim _{r \rightarrow 1} \int_{\mathbb{T}} \int_{\mathbb{T}} \frac{1}{1-r^{2} \varepsilon \bar{\zeta}} \overline{g(\varepsilon)} g(\zeta) d \sigma(\varepsilon) d \sigma(\eta) .
$$

Since by previous lemma we have (4.19) by (4.18), these imply

$$
\left\|P_{+}\left(\chi_{\Lambda} g\right)\right\|^{2}-\left\|P_{+}\left(\bar{\phi} \chi_{\Lambda} g\right)\right\|^{2} \geq 0 .
$$

Then it follows by (4.22) that

$$
\lim _{r \rightarrow 1} \int_{\Lambda} \int_{\Lambda}\left(\frac{1-\phi(\xi) \overline{\phi(\zeta)}}{1-r^{2} \xi \bar{\zeta}}\right) \overline{g(\xi)} g(\zeta) d \sigma(\xi) d \sigma(\zeta) \geq 0 \quad \text { for all } g \in L^{2}(\mathbb{T}) \text {. }
$$




\section{Appendix A}

\section{Hilbert Spaces}

\section{A.1 Definitions}

Definition A.1.1. Let $\mathcal{H}$ be a linear space over the complex field $\mathbb{C}$. An inner product on $\mathcal{H}$ is a function $\langle\cdot, \cdot\rangle: \mathcal{H} \times \mathcal{H} \rightarrow \mathbb{C}$ which satisfies the following conditions:

(i) $\langle f, g\rangle=\overline{\langle g, f\rangle}$ for $f, g \in \mathcal{H}$.

(ii) $\langle\lambda f+\mu g, h\rangle=\lambda\langle f, h\rangle+\mu\langle g, h\rangle$ and

$$
\langle f, \lambda g+\mu h\rangle=\bar{\lambda}\langle f, g\rangle+\bar{\mu}\langle f, h\rangle \quad \text { for } \quad \lambda, \mu \in \mathbb{C} \quad \text { and } \quad f, g \text { and } h \in \mathcal{H} .
$$

(iii) $\langle f, f\rangle \geq 0$ for $f \in \mathcal{H}$ and $\langle f, f\rangle=0 \quad$ if and only if $f=0$.

Definition A.1.2. A pre-Hilbert space (inner product space) $\mathcal{H}$ is a linear space over $\mathbb{C}$ with an inner product defined on it.

If $\mathcal{H}$ is an inner product space, $\|\cdot\|$ defines a norm on $\mathcal{H}$ by

$$
\|f\|=\langle f, f\rangle^{1 / 2} .
$$

Sometimes we use the notations $\|\cdot\|_{\mathcal{H}}$ and $\langle\cdot, \cdot\rangle_{\mathcal{H}}$ for norm and inner product, respectively. 
Some important properties:

$$
\begin{gathered}
\|f\| \geq 0 \text { and equality occurs only when } f=0 . \\
\|\xi f\|=|\xi|\|f\| \text { for } f \in \mathcal{H}, \quad \xi \in \mathbb{C} \text {. } \\
|\langle f, g\rangle| \leq\|f\|\|g\| \quad \text { for } \quad f, g \in \mathcal{H} \quad \text { (Schwarz Inequality), }
\end{gathered}
$$

and equality occurs when $f$ and $g$ are linearly dependent.

$$
\|f+g\| \leq\|f\|+\|g\| \quad \text { for } f, g \in \mathcal{H} \text { (Triangular Inequality), }
$$

and equality occurs when $f$ and $g$ are linearly dependent.

$$
\langle f, g\rangle=\frac{1}{4}\left(\|f+g\|^{2}-\|f-g\|^{2}+i\|f+i g\|^{2}-\|f-i g\|^{2}\right) \quad \text { for } \quad f, g \in \mathcal{H}
$$

(polarization identity).

$$
\|f+g\|^{2}+\|f-g\|^{2}=2\|f\|^{2}+2\|g\|^{2} \text { for } f, g \in \mathcal{H} \text { (parallelogram identity). }
$$

Define $d(f, g):=\|f-g\|$ the binary function. Observe that for $f, g$ and $h \in \mathcal{H}$ and for all $\xi \in \mathbb{C}, d$ satisfies all requirements to be a metric:

(i) $d(f, g) \geq 0$ and equality occurs if and only if $f=g$.

(ii) $d(f, g)=d(g, f)$

(iii) $d(f, g) \leq d(f, h)+d(h, g)$.

(iv) $d(f-h, g-h)=d(f, g) \quad$ (translation invariant).

(v) $d(\xi f, \xi g)=|\xi| \cdot d(f, g)$.

This binary function introduces metric topology in $\mathcal{H}$ which is called strong topology or norm topology.

In other words a sequence $(f)_{n \geq 0}$ in $\mathcal{H}$ converges strongly to $\mathrm{f}$ if $\left\|f_{n}-f\right\| \rightarrow$ 0 as $n \rightarrow \infty$. 
Definition A.1.3. A map $L$ from a linear space to another is called linear operator if it satisfies

$$
L(\eta f+\zeta g)=\eta L f+\zeta L g
$$

for $\eta, \zeta \in \mathbb{C}$ and $f, g \in \mathcal{H}$.

The operator $L$ is continuous if $L$ is continuous at each point of domain of $L$. To be able to speak of continuity of a linear operator, the domain and range spaces have to be equipped with respective topologies.

Let $L$ be a linear operator from $\mathcal{H}$ to $\mathcal{G}$ with $\mathcal{H}$ and $\mathcal{G}$ are pre-Hilbert spaces. The Lipschitz constant for a linear operator $L$ is called its norm and denoted by $\|L\|$ where

$$
\|L\|=\sup \left\{\|L f\|_{\mathcal{G}} /\|f\|_{\mathcal{H}}: 0 \neq f \in \mathcal{H}\right\}
$$

Theorem A.1.4. Let $L$ be a linear operator from $\mathcal{H}$ to $\mathcal{G}$ where $\mathcal{H}$ and $\mathcal{G}$ are pre-Hilbert spaces. The followings are mutually equivalent:

(i) L is continuous

(ii) $L$ is bounded in the sense that

$$
\sup \left\{\|L f\|_{G}:\|f\|_{H} \leq \sigma\right\}<\infty
$$

for $0 \leq \sigma<\infty$.

(iii) $L$ is Lipschitz continuous in the sense that there is $0 \leq \gamma<\infty$ such that

$$
\|L f-L g\|_{G} \leq \gamma\|f-g\|_{\mathcal{H}}
$$

for $f, g \in \mathcal{H}$.

Proof. (i) $\Longrightarrow$ (ii)

Let $L$ be continuous. Define a neighborhood of 0 in $\mathcal{G}$ as $v=\left\{g:\|g\|_{\mathcal{G}} \leqq 1\right\}$. Continuity of $L$ implies $L 0=0$ and there is a neighborhood (of 0 in $\mathcal{H}$ ) of the form $u=\left\{f:\|f\|_{\mathcal{H}} \leqq \delta\right\}$ which is mapped into $v$ by $L$, where $\delta>0$. Now let 
us take a nonzero $f \in \mathcal{H}$ such that $\|f\|_{\mathcal{H}} \leqq \rho$, for $\rho>0$. Since $\|\delta f / \rho\|_{\mathcal{H}} \leq \delta$, then $\frac{\delta f}{\rho} \in u$. As $L$ is linear,

$$
\delta\|L f\|_{\mathcal{G}} / \rho=\|L(\delta f / \rho)\|_{\mathcal{G}} \leqq 1
$$

and

$$
\sup \left\{\|L f\|_{\mathcal{G}}:\|f\|_{\mathcal{H}} \leqq \rho\right\} \leqq \frac{\rho}{\delta} .
$$

(ii) $\Longrightarrow$ (iii)

Let $L$ satisfies (ii). Consider the norm of $L f-L g$. By linearity property of $L$,

$$
\|L f-L g\|_{G}=\|L(f-g)\|_{\mathcal{G}} \leqq \gamma\|f-g\|_{\mathcal{H}} \quad \text { for } \quad f, g \in \mathcal{H},
$$

where $\gamma$ is the supremum in (ii) with $\rho=1$.

$$
\text { (iii) } \Longrightarrow \text { (i) }
$$

This is obvious since boundedness implies continuity.

Denote by $B(\mathcal{H}, \mathcal{G})$, all continuous linear operators from $\mathcal{H}$ to $\mathcal{G}, \mathcal{H}$ and $\mathcal{G}$ are pre-Hilbert spaces. $B(\mathcal{H}, \mathcal{G})$ becomes a linear space with respect to the natural addition and scalar multiplication:

$$
(\xi L+\zeta M) f=\xi L f+\zeta M f,
$$

where $L$ and $M$ are linear operators, $f \in \mathcal{H}$ and $\xi \in \mathbb{C}$.

When $\mathcal{H}=\mathcal{G}, B(\mathcal{H}, \mathcal{G})$ is denoted by $B(\mathcal{H})$.

Let $\mathcal{K}$ be another pre-Hilbert space. If $L \in B(\mathcal{H}, \mathcal{G})$ and $K \in B(\mathcal{G}, \mathcal{K})$, then their product will be $K L \in B(\mathcal{H}, \mathcal{K})$ and defined by

$$
(K L) f=K(L f) \quad \text { for } \quad f \in \mathcal{H} \text {. }
$$

Moreover we have the following properties:

(i) $K(\xi L+\zeta M)=\xi K L+\zeta K M$ 
(ii) $\|\xi L\|=|\xi| \cdot\|L\|$

(iii) $\|L+M\| \leq\|L\|+\|M\|$ and

(iv) $\|K L\| \leq\|K\|\|L\|$.

For $\mathcal{H}, \mathcal{G}$ are pre-Hilbert spaces and $L, M$ are linear operators, $B(\mathcal{H}, \mathcal{G})$ becomes a metric space with respect to the translation invariant, positively homogenous distance function

$$
d(L, M):=\|L-M\| .
$$

For each $K \in B(\mathcal{G}, \mathcal{K})$, the map $L \longmapsto K L$ becomes a continuous linear operator from $B(\mathcal{H}, \mathcal{G})$ to $B(\mathcal{H}, \mathcal{K})$.

Definition A.1.5. A linear operator from $\mathcal{H}$ to the scalar field $\mathbb{C}$ is called a linear functional (or linear form).

Definition A.1.6. A pre-Hilbert space $\mathcal{H}$ is called a Hilbert space if it is complete in metric. In other words, if $f_{n}$ is a Cauchy sequence in $\mathcal{H}$, i.e. $\left\|f_{n}-f_{m}\right\| \longrightarrow 0$ when $n, m \rightarrow \infty$, then there is $f \in \mathcal{H}$ such that $\left\|f_{n}-f\right\| \longrightarrow 0$ when $n \rightarrow \infty$.

Every subspace of a pre-Hilbert space is again a pre-Hilbert space with respect to the induced inner product; but for a subspace of a Hilbert space to be again a Hilbert space, it has to be closed.

Let $\mathcal{H}$ be a pre-Hilbert space. We can imbed $\mathcal{H}$ as a dense subspace of a Hilbert space $\tilde{\mathcal{H}}$ in such a way that

$$
\langle f, g\rangle_{\tilde{\mathcal{H}}}=\langle f, g\rangle_{\mathcal{H}} \quad \text { for } f, g \in \mathcal{H}
$$

This canonical method is called completion. If we have a continuous linear operator $L$ from a dense subspace $\mathcal{M}$ of a Hilbert space $\mathcal{H}$ to a Hilbert space $\mathcal{G}$, we can extend $L$ uniquely to a continuous linear operator from $\mathcal{H}$ to $\mathcal{G}$ with preserving norm.

Theorem A.1.7. Let $\mathcal{M}$ be a dense subspace of a Hilbert space $\mathcal{H}$, and $\mathcal{N}$ a dense subspace of a Hilbert space $\mathcal{G}$. If a linear operator $L$ from $\mathcal{M}$ to $\mathcal{G}$ satisfies 


$$
\left|\langle L f, g\rangle_{\mathcal{G}}\right| \leq|\gamma|\|f\|_{\mathcal{H}}\|g\|_{\mathcal{G}} \quad \text { for } \quad f \in \mathcal{H}, \quad g \in \mathcal{M} \quad \text { with some } 0 \leq \gamma<\infty
$$

then it is uniquely extended to a continuous linear operator from $\mathcal{M}$ to $\mathcal{G}$ with norm $\leq \gamma$. The minimum of such $\gamma$ coincides with norm.

Proof. Let $L$ be a linear operator from $M$ to $\mathcal{G}$ and satisfies (A.8). By fixing $f$, since $g \longmapsto\langle L f, g\rangle_{\mathcal{G}}$ and $g \longmapsto\|g\|_{\mathcal{G}}$ are continuous, we can assume that (A.8) is satisfied for all $g \in \mathcal{G}$.

Now by using (A.8) and Schwarz inequality, we get

$$
\|L f\|_{\mathcal{G}}^{2}=\langle L f, L f\rangle_{\mathcal{G}} \leq \gamma\|f\|_{\mathcal{H}}\|L f\|_{\mathcal{G}}
$$

which implies

$$
\|L f\|_{\mathcal{G}} \leq \gamma\|f\|_{\mathcal{H}} \quad \text { for all } f \in M
$$

Hence $L$ is continuous operator from $M$ to $\mathcal{G}$ with norm $\leq \gamma$. So we can extend $L$ uniquely to a continuous linear operator from $\mathcal{H}$ to $\mathcal{G}$ with preserving norm. It is obvious that minimum of $\gamma$ coincides with norm.

Theorem A.1.8. Let $L^{2}(\Omega, \mu)$ consists of all measurable functions $f(\omega)$ on $\Omega$ such that

$$
\int_{\Omega}|f(\omega)|^{2} d \mu(\omega)<\infty,
$$

where $(\Omega, \mu)$ is a measure space such that $\Omega$ is the union of subsets of finite positive measure. Then $L^{2}(\Omega, \mu)$ becomes a Hilbert space with respect to the inner product

$$
\langle f, g\rangle:=\int_{\Omega} f(\omega) \overline{g(\omega)} d \mu(\omega)
$$

Proof. We have

$$
|f(\omega)+g(\omega)|^{2} \leq 2\left\{|f(\omega)|^{2}+|g(\omega)|^{2}\right\}
$$

which implies that $L^{2}(\Omega, \mu)$ is a linear space. If we expand the left handside, we have

$$
|f(\omega)+g(\omega)|^{2}=|f(\omega)|^{2}+|g(\omega)|^{2}+2 f(\omega) g(\omega) \leq 2\left\{|f(\omega)|^{2}+|g(\omega)|^{2}\right\}
$$


which implies that

$$
\mid f(\omega) \overline{g(\omega) \mid} \leq \frac{1}{2}\left\{|f(\omega)|^{2}+|g(\omega)|^{2}\right\} .
$$

So, $\langle f, g\rangle$ in (A.10) is well-defined and the linear space $L^{2}(\Omega, \mu)$ becomes a pre-Hilbert space.

Completeness:

Let us take a Cauchy sequence $\left(f_{n}\right)_{n \geq 1}$ in $L^{2}(\Omega, \mu)$. Suppose that

$$
\sum_{n=1}^{\infty}\left\|f_{n+1}-f_{n}\right\| \equiv \gamma<\infty
$$

For any $\mathrm{m}$, consider the following integration,

$$
\int_{\Omega} \sum_{n=1}^{m}\left|f_{n+1}(\omega)-f_{n}(\omega)\right|^{2} d \mu(\omega) \leq \sum_{n=1}^{\infty}\left\|f_{n+1}-f_{n}\right\|^{2}=\gamma^{2}<\infty .
$$

Then by the Lebesque theorem,

$$
\int_{\Omega} \sum_{n=1}^{\infty}\left|f_{n+1}(\omega)-f_{n}(\omega)\right|^{2} d \mu(\omega) \leq \gamma^{2}<\infty
$$

which implies

$$
g(\omega) \equiv \sum_{n=1}^{m}\left|f_{n+1}(\omega)-f_{n}(\omega)\right|<\infty \quad \text { for almost all } \omega,
$$

and

$$
\int_{\Omega} g(\omega)^{2} d \mu(\omega)=\int_{\Omega} \sum_{n=1}^{\infty}\left|f_{n+1}(\omega)-f_{n}(\omega)\right|^{2} d \mu(\omega) \leq \gamma^{2}<\infty .
$$

Hence for almost all $\omega$, the sequence $\left(f_{n}(\omega)\right)_{n \geq 1}$ converges. To find the limit of $f(\omega)$, consider the following

$$
\begin{aligned}
\left|f_{n+1}(\omega)-f_{n}(\omega)\right|^{2} & \leq \sum_{k=n}^{\infty}\left|f_{k+1}(\omega)-f_{k}(\omega)\right|^{2} \\
& \leq \sum_{n=1}^{m}\left|f_{n+1}(\omega)-f_{n}(\omega)\right|=g(\omega)
\end{aligned}
$$

and again by Lebesque theorem,

$$
\begin{aligned}
\lim _{n \rightarrow \infty}\left\|f_{n}-f\right\|^{2} & =\lim _{n \rightarrow \infty}\left\langle f_{n}-f, f_{n}-f\right\rangle=\lim _{n \rightarrow \infty} \int_{\Omega}\left|f_{n}(\omega)-f(\omega)\right|^{2} d \mu(\omega) \\
& \leq \int_{\Omega} \lim _{n \rightarrow \infty}\left|f_{n}(\omega)-f(\omega)\right|^{2} d \mu(\omega)=0 .
\end{aligned}
$$


Therefore $f_{n}$ converges strongly to $f$, which means $L^{2}(\Omega, \mu)$ is complete and it is a Hilbert space with respect to the given inner product.

Let $\mathcal{H}$ and $\mathcal{G}$ be Hilbert spaces. The algebraic direct sum of $\mathcal{H}$ and $\mathcal{G}$, consisting of $f \oplus g$ with the functions $f \in \mathcal{H}$ and $g \in \mathcal{G}$, becomes a Hilbert space, denoted by $\mathcal{H} \oplus \mathcal{G}$ with respect to the inner product

$$
\left\langle f \oplus g, f^{\prime} \oplus g^{\prime}\right\rangle=\left\langle f, f^{\prime}\right\rangle_{\mathcal{H}}+\left\langle g, g^{\prime}\right\rangle_{\mathcal{G}}
$$

Let $\mathcal{H}$ and $\mathcal{G}$ be Hilbert spaces. The algebraic tensor product of $\mathcal{H}$ and $\mathcal{G}$, consisting of finite sums of $f \otimes g$ with $f \in \mathcal{H}$ and $g \in \mathcal{G}$, becomes a pre-Hilbert space with respect to the inner product

$$
\left\langle\sum_{i} f_{i} \otimes g_{i}, \sum_{j} f_{j}^{\prime} \otimes g_{j}^{\prime}\right\rangle=\sum_{i, j}\left\langle f_{i}, f_{j}^{\prime}\right\rangle_{\mathcal{H}} \cdot\left\langle g_{i}, g_{j}^{\prime}\right\rangle_{\mathcal{G}} .
$$

Definition A.1.9. The canonical completion of the algebraic tensor product of $\mathcal{H}$ and $\mathcal{G}$, where $\mathcal{H}$ and $\mathcal{G}$ are Hilbert spaces, is called tensor product Hilbert space and denoted by $\mathcal{H} \otimes \mathcal{G}$.

\section{A.2 Projection}

We start this section with an important theorem.

Theorem A.2.1 (F.Riesz). For each continuous linear functional $\phi$ on a Hilbert space $\mathcal{H}$, there exists uniquely $g \in \mathcal{H}$ such that

$$
\phi(f)=\langle f, g\rangle \text { for } f \in \mathcal{H} .
$$

Proof. For the case $\phi=0$, take $g=1$, then we are done.

Let $\phi \neq 0$. For this case, we can suppose that $\|\phi\|=1$. Then there exists a sequence $g_{n}$ such that

$$
\left\|g_{n}\right\|=1 \quad(n=1,2, . .) \quad \text { and } \quad \phi\left(g_{n}\right) \longrightarrow\|\phi\|=1 \text { as } n \rightarrow \infty .
$$


Then by parallelogram rule

$$
\left\|g_{m}-g_{n}\right\|^{2}+\left\|g_{m}+g_{n}\right\|^{2}=2\left\|g_{m}\right\|^{2}+2\left\|g_{n}\right\|^{2}=2+2=4
$$

On the other hand by triangular rule,

$$
2=\left\|g_{m}\right\|+\left\|g_{n}\right\| \geq\left\|g_{m}+g_{n}\right\|
$$

then we get

$$
2 \geq\left\|g_{m}+g_{n}\right\| \geq\left|\phi\left(g_{m}\right)+\phi\left(g_{n}\right)\right| \longrightarrow 2 \text { as } m, n \rightarrow \infty
$$

Thus,

$$
\left\|g_{m}+g_{n}\right\|=2 \text { and }\left\|g_{m}+g_{n}\right\|^{2}=4 \text {. So, }\left\|g_{m}-g_{n}\right\| \longrightarrow 0 \quad \text { as } \quad m, n \rightarrow \infty \text {. }
$$

Then, due to the metric completeness, $g_{n}$ converges strongly to some $g$ such that $\|g\|=\phi(g)=1$.

Let $f \in \mathcal{H}$ be arbitrary. If $\langle f, g\rangle=0$, then for all $\zeta \in \mathbb{C}$,

$$
\|g+\zeta f\|^{2}=\|g\|^{2}+\bar{\zeta}\langle g, f\rangle+\zeta\langle f, g\rangle+|\zeta|^{2}\|f\|^{2}=1+|\zeta|^{2}\|f\|^{2}
$$

and

$$
\begin{aligned}
\|g+\zeta f\|^{2} & \geq|\phi(g+\zeta f)|^{2}=|\phi(g)+\zeta \phi(f)|^{2}=|1+\zeta \phi(f)|^{2} \\
& =1+2 \operatorname{Re} \zeta \phi(f)+|\zeta|^{2}|\phi(f)|^{2} .
\end{aligned}
$$

Combining these equations, we get

$$
1+|\zeta|^{2}\|f\|^{2} \geq 1+2 \operatorname{Re} \zeta \phi(f)+|\zeta|^{2}|\phi(f)|^{2}
$$

Since $\zeta$ is arbitrary, then $\phi(f)=0$. This implies

$$
\phi(f)=\langle f, g\rangle \quad \text { for } \quad f \text { satisfying }\langle f, g\rangle=0 \text {. }
$$

Now consider the other case which is $\langle f, g\rangle \neq 0$. Define $h:=f-\langle f, g\rangle g$. It follows that,

$$
\langle h, g\rangle=\langle f-\langle f, g\rangle g, g\rangle=\langle f, g\rangle-\langle\langle f, g\rangle g, g\rangle=\langle f, g\rangle-\langle f, g\rangle=0 .
$$


Then,

$$
0=\phi(h)=\phi(f)-\langle f, g\rangle \phi(g)=\phi(f)-\langle f, g\rangle .
$$

Hence we obtain $\phi(f)=\langle f, g\rangle$.

To see the uniqueness of $g$, suppose that there exists $g^{\prime} \in \mathcal{H}$ such that for each linear functional $\phi$, we have

$$
\phi(f)=\left\langle f, g^{\prime}\right\rangle .
$$

Also this equality is satisfied for $g$. Thus we have

$$
\phi(f)=\left\langle f, g^{\prime}\right\rangle=\langle f, g\rangle
$$

which implies

$$
\left\langle f, g-g^{\prime}\right\rangle=0 .
$$

Hence $g=g^{\prime}$.

Theorem A.2.2. For any closed subspace $\mathcal{M}$ of a Hilbert space $\mathcal{H}$,

$$
\mathcal{H}=\mathcal{M} \oplus \mathcal{M}^{\perp} \text { (algebraic direct sum), }
$$

that is, each $f \in \mathcal{H}$ can be uniquely written in the form

$$
f=f_{\mathcal{M}}+f_{\mathcal{M}^{\perp}} \text { with } f_{\mathcal{M}} \in \mathcal{M}, f_{\mathcal{M}^{\perp}} \in \mathcal{M}^{\perp} .
$$

Moreover, $\left\|f_{\mathcal{M}}\right\|$ coincides with the distance from $f$ to $\mathcal{M}^{\perp}$;

$$
\left\|f_{\mathcal{M}}\right\|=\min \left\{\|f-g\|: g \in \mathcal{M}^{\perp}\right\}
$$

the distance being attained only at $f_{\mathcal{M}^{\perp}}$.

Proof. Since $\mathcal{M}$ is closed, it is also a Hilbert space. By Riesz theorem to the continuous functional $h \longmapsto\langle h, g\rangle$ restricted to $\mathcal{M}$, there is $f_{\mathcal{M}} \in \mathcal{M}$, such that

$$
\langle h, f\rangle=\left\langle h, f_{\mathcal{M}}\right\rangle \text { for } h \in \mathcal{M} .
$$

By this, we get $\left\langle h, f-f_{M}\right\rangle=0$. Let us define $f_{\mathcal{M}^{\perp}}:=f-f_{\mathcal{M}}$. Since $\left\langle h, f-f_{\mathcal{M}}\right\rangle=0$, then $f_{\mathcal{M}^{\perp}} \perp h \quad$ for all $h \in \mathcal{M}$. So, $f_{\mathcal{M}^{\perp}} \in \mathcal{M}^{\perp}$. Hence

$$
f=f_{\mathcal{M}}+f_{\mathcal{M}^{\perp}} \quad \text { where } \quad f_{\mathcal{M}} \in \mathcal{M}, f_{\mathcal{M}^{\perp}} \in \mathcal{M}^{\perp} .
$$


For the uniqueness, let $f=f_{\mathcal{M}}^{\prime}+f_{\mathcal{M}^{\perp}}^{\prime}$ be any other decomposition of $f$, where $f_{\mathcal{M}}^{\prime} \in \mathcal{M}, f_{\mathcal{M}^{\perp}}^{\prime} \in \mathcal{M}^{\perp}$. Then,

$$
f=f_{\mathcal{M}}^{\prime}+f_{\mathcal{M}^{\perp}}^{\prime}=f_{\mathcal{M}}+f_{\mathcal{M}^{\perp}}
$$

which implies that

$$
f_{\mathcal{M}}-f_{\mathcal{M}}^{\prime}=f_{\mathcal{M}^{\perp}}-f_{\mathcal{M}^{\perp}}^{\prime}
$$

and by the last equality we obtain that $f_{\mathcal{M}}=f_{\mathcal{M}}^{\prime}$.

For the moreover part, let us take any $g \in \mathcal{M}^{\perp}$. Since $\left\langle f_{\mathcal{M}^{\perp}}-g, f\right\rangle=0$, we get

$$
\|f-g\|^{2}=\left\|f_{\mathcal{M}}+f_{\mathcal{M}^{\perp}}-g\right\|^{2}=\left\|f_{\mathcal{M}}\right\|^{2}+\left\|f_{\mathcal{M}^{\perp}}-g\right\|^{2} .
$$

Then we have

$$
\|f-g\|^{2} \geq\left\|f_{\mathcal{M}}\right\|^{2} \quad \text { and equality occurs when } g=f_{\mathcal{M}^{\perp}} \text {. }
$$

Hence,

$$
\left\|f_{\mathcal{M}}\right\|=\min \left\{\|f-g\|: g \in \mathcal{M}^{\perp}\right\} .
$$

Corollary A.2.3. In a Hilbert space, the closed linear span of any subset $A$ coincides with $\left(A^{\perp}\right)^{\perp}$.

Proof. Let $\mathcal{M}$ be the closed linear span of $A$. So, we have $\mathcal{M} \subseteq\left(A^{\perp}\right)^{\perp}$. It remains to show the other side of inclusion. As $\left(A^{\perp}\right)^{\perp}$ is a closed subspace of $\mathcal{H}$, so it is also a Hilbert space. Now by using the Theorem A.2.2, for each $f \in\left(A^{\perp}\right)^{\perp}, f$ has a decomposition such that $f=g+h$ where $g \in \mathcal{M}, h \in$ $\left(A^{\perp}\right)^{\perp}$ and $h$ is orthogonal to all of $\mathcal{M}$. Since $A \subseteq \mathcal{M}, h$ must be orthogonal to all of $A$. In other words, $h$ must be in $A^{\perp}$. But $h$ is both in $A^{\perp}$ and $\left(A^{\perp}\right)^{\perp}$. Hence $h=0$ and $h=0=f-g$ implies $f=g \in \mathcal{M}$ i.e. $\left(A^{\perp}\right)^{\perp} \subseteq \mathcal{M}$. Consequently, $\mathcal{M}=\left(A^{\perp}\right)^{\perp}$.

Definition A.2.4. A subset of a Hilbert space $\mathcal{H}$ is said to be total (complete) in $\mathcal{H}$ if 0 is the only element that is orthogonal to all of $A$, i.e. $A^{\perp}=\{0\}$. 
So by the Corollary A.2.3, $A$ is total if and only if every element of $\mathcal{H}$ can be approximated by linear combinations of elements of $A$.

Definition A.2.5. When $\mathcal{M}$ is a closed subspace of $\mathcal{H}$, the map $f \longmapsto f_{\mathcal{M}}$ determines a linear operator from $\mathcal{H}$ to $\mathcal{M}$ with norm $\leq 1$. This operator is called the orthogonal projection to $\mathcal{M}$ and is denoted by $P_{\mathcal{M}}$.

If $I$ is the identity operator, then $I-P_{\mathcal{M}}$ becomes the orthogonal projection to $\mathcal{M}^{\perp}$ and

$$
\|f\|^{2}=\left\|P_{\mathcal{M}} f\right\|^{2}+\left\|\left(I-P_{\mathcal{M}}\right) f\right\|^{2}
$$

Theorem A.2.6 (Abstract Interpolation). Let $\mathcal{H}$ be a Hilbert space, $X$ an index set and $\gamma$ a positive number. Given a map $x \longmapsto f_{x}$ from $X$ to $\mathcal{H}$ and one $x \longmapsto a_{x}$ from $X$ to $\mathbb{C}$, there exists an element $g \in \mathcal{H}$ such that

$$
\|g\| \leq \gamma \quad \text { and } \quad\left\langle f_{x}, g\right\rangle=a_{x} \quad \text { for } \quad x \in X
$$

if and only if

$$
\sum_{x, y} \overline{\xi_{y}} \xi_{x} \overline{a_{y}} a_{x} \leq \gamma^{2} \sum_{x, y} \overline{\xi_{y}} \xi_{x}\left\langle f_{x}, f_{y}\right\rangle \quad \text { for } \quad\left[\xi_{x}\right]
$$

where $\left[\xi_{x}\right]$ denotes, in general, a function on $X$ which vanishes outside a finite number of points.

Proof. Suppose that $(\mathrm{A} .15)$ is satisfied for $g \in \mathcal{H}$. Then

$$
\sum_{x, y} \overline{\xi_{y}} \xi_{x} \overline{a_{y}} a_{x}=\left|\sum_{x} \xi_{x} a_{x}\right|^{2}=\left|\sum_{x} \xi_{x}\left\langle f_{x}, g\right\rangle\right|^{2}=\left|\left\langle\sum_{x} \xi_{x} f_{x}, g\right\rangle\right|^{2}
$$

by the Schwarz inequality

$$
\leq\left\|\sum_{x} \xi_{x} f_{x}\right\|^{2}\|g\|^{2} \leq \gamma^{2}\left\|\sum_{x} \xi_{x} f_{x}\right\|^{2}=\gamma^{2} \sum_{x, y} \overline{\xi_{y}} \xi_{x}\left\langle f_{x}, f_{y}\right\rangle .
$$

So (A.16) is satisfied.

Now suppose (A.16) is satisfied. Then the correspondence

$$
\sum_{x} \xi_{x} f_{x} \longmapsto \sum_{x} \xi_{x} a_{x}
$$


defines a linear functional on the linear span of $\left\{f_{x}: x \in X\right\}$ with norm $\leq \gamma$. This bounded functional can be extended to the closure of this linear span, and then by using Riesz's Representation Theorem (see Theorem A.2.1) the vector $g$ can be found such that $\|g\| \leq \gamma$ and $\left\langle f_{x}, g\right\rangle=a_{x}$ for all $x \in X$.

\section{A.3 Weak Topology}

Definition A.3.1. The weak topology of a Hilbert space $\mathcal{H}$ is the weakest topology that makes continuous all linear functionals of the form $f \longmapsto\langle f, g\rangle$.

For an element $f$ in the weak topology, a fundamental system of neighborhoods of $f$ consists of subsets of the form

$$
U(f ; A, \epsilon)=\{h:|\langle f, g\rangle-\langle h, g\rangle|<\epsilon \text { for } g \in A\},
$$

where $A$ is a finite subset of $H$ and $\epsilon$ is a positive number. Then a directed net $\left\{f_{\lambda}\right\}$ converges weakly to $f$ if and only if

$$
\left\langle f_{\lambda}, g\right\rangle \stackrel{\lambda}{\longrightarrow}\langle f, g\rangle
$$

for all $g \in \mathcal{H}$.

Definition A.3.2. The (operator) weak topology in the space $B(\mathcal{H}, \mathcal{G})$ of continuous linear operators from $\mathcal{H}$ to a Hilbert space $\mathcal{G}$, is the weakest topology that makes continuous all linear functionals of the form $L \longmapsto\langle L f, g\rangle$ for $f \in \mathcal{H}$ and $g \in \mathcal{G}$.

Then a directed net $\left\{L_{\lambda}\right\}$ converges (operator) weakly to $L$ if and only if

$$
\left\langle L_{\lambda} f, g\right\rangle \stackrel{\lambda}{\longrightarrow}\langle L f, g\rangle .
$$

Definition A.3.3. The (operator) strong topology is the weakest topology that makes continuous all linear operators of the form $L \longmapsto L f$ for $f \in \mathcal{H}$. 
Then a directed net $\left\{L_{\lambda}\right\}$ converges (operator) strongly to $L$ if and only if

$$
\left\|L_{\lambda} f-L f\right\| \stackrel{\lambda}{\longrightarrow} 0
$$

for all $f \in \mathcal{H}$.

Theorem A.3.4 ([1]). The closed unit ball $U:=\{f:\|f\| \leq 1\}$ of a Hilbert space $\mathcal{H}$ is weakly compact.

Theorem A.3.5 ([1]). The closed unit ball $\{L:\|L\| \leq 1\}$ of $B(\mathcal{H}, \mathcal{G})$ is (operator) weakly compact.

Theorem A.3.6. If a subset $A$ of a Hilbert space $\mathcal{H}$ is weakly bounded in the sense

$$
\sup _{f \in A}|\langle f, g\rangle|<\infty \text { for } g \in \mathcal{H},
$$

then it is strongly bounded, that is, $\sup _{f \in A}\|f\|<\infty$.

Proof. Define

$$
B_{n}:=\{g:|\langle f, g\rangle| \leq n \text { for } f \in A\}, \quad(n=1,2,3, \ldots) .
$$

Then for each $n, B_{n}$ will be a closed subset of $\mathcal{H}$ and so $\mathcal{H}=\bigcup_{n} B_{n}$. By Baire category theorem, since $\mathcal{H}$ is a complete metric space with respect to the strong topology and $\mathcal{H}$ is covered by a countable number of closed sets, then at least one of them has nonempty interior, say $B_{n_{0}}$. So, for some $g_{0}$ and $\epsilon>0$,

$$
V \equiv\left\{g:\left\|g-g_{0}\right\| \leq \epsilon\right\} \subset B_{n_{0}}
$$

Let $g$ be in $\mathcal{H}$. Since

$$
\left\|g_{0}+\epsilon g /\right\| g\left\|-g_{0}\right\|=\||\epsilon| g /\| g\|\| \leq \epsilon
$$

then $g_{0}+\epsilon g /\|g\|$ will be in $V$. Then

$$
\epsilon|\langle f, g\rangle| /\|g\| \leq\left|\left\langle f, g_{0}+\epsilon g /\|g\|\right\rangle\right|+\left|\left\langle f, g_{0}\right\rangle\right| \leq n_{0}+n_{0}=2 n_{0} \quad \text { for } \quad f \in A .
$$

This implies,

$$
|\langle f, g\rangle| \leq 2 n_{0}\|g\| / \epsilon \quad \text { for } \quad f \in A \text {. }
$$


Putting $f$ instead of $g$ in the last inequality and using Schwarz inequality, we get

$$
\sup _{f \in A} \leq 2 n_{0} / \epsilon<\infty .
$$

Theorem A.3.7. Let $\mathcal{H}$ and $\mathcal{G}$ be Hilbert spaces. For a linear operator $L$ from $\mathcal{H}$ to $\mathcal{G}$, strong continuity and weak continuity are the same thing.

Proof. Let $L$ be weakly continuous. Since by Theorem A.3.4 the unit ball $U$ of a Hilbert space is weakly compact, it follows that $L(U)$ is also weakly compact and so weakly bounded in $G$. Since Theorem A.3.6 states that weakly boundedness implies strongly boundedness, $L(U)$ will be strongly bounded and hence it is strongly continuous by TheoremA.1.4

Now suppose that $L$ is strongly continuous. So we have, for each $g \in \mathcal{G}$, the linear functional $f \longmapsto\langle L f, g\rangle_{\mathcal{G}}$ is continuous on H. Now by Riesz theorem, there is $g^{\prime} \in \mathcal{H}$ such that,

$$
f \longmapsto\left\langle f, g^{\prime}\right\rangle_{\mathcal{H}} \text { for } f \in \mathcal{H},
$$

then

$$
\langle L f, g\rangle_{\mathcal{G}}=\left\langle f, g^{\prime}\right\rangle_{\mathcal{H}} \text { for } f \in \mathcal{H} .
$$

Hence $L$ is weakly continuous.

Theorem A.3.8 ([1]). Let $\mathcal{H}$ and $\mathcal{G}$ be Hilbert spaces. Then the following assertions for a subset $\mathbf{L}$ of $B(\mathcal{H}, \mathcal{G})$ are mutually equivalent:

(i) $\mathbf{L}$ is (operator) weakly bounded;

$$
\sup _{L \in \mathbf{L}}|\langle L f, g\rangle|<\infty \text { for } f \in \mathcal{H}, \quad g \in \mathcal{G} .
$$

(ii) $\mathbf{L}$ is (operator) strongly bounded;

$$
\sup _{L \in \mathbf{L}}\|L f\|<\infty \text { for } f \in \mathcal{H} .
$$

(iii) $\mathbf{L}$ is norm bounded (or uniformly bounded);

$$
\sup _{L \in \mathbf{L}}\|L\|<\infty .
$$


Definition A.3.9. A linear operator $L$ from $\mathcal{H}$ to $\mathcal{G}$, where $\mathcal{H}$ and $\mathcal{G}$ are Hilbert spaces, is said to be closed if its graph

$$
G_{L}:=\{f \oplus L f: f \in \mathcal{H}\}
$$

is a closed subspace of the direct sum space $\mathcal{H} \oplus \mathcal{G}$, that is, if $\mid f_{n}-f \| \longrightarrow 0$ in $\mathcal{H}$ as $(n \rightarrow \infty)$ and $\left\|L f_{n}-g\right\| \longrightarrow 0$ in $\mathcal{G}$ as $(n \rightarrow \infty)$, then $g=L f$.

Theorem A.3.10. Any closed linear operator $L$ from a Hilbert space $\mathcal{H}$ to another Hilbert space $\mathcal{G}$ is continuous.

Proof. Let $G_{L}$ be the graph of $L$. Since $L$ is closed, then $G_{L}$ is a closed subspace and so it is a Hilbert space. Consider the linear operator $\phi: f \oplus L f \longmapsto f$ from $G_{L}$ to $\mathcal{H}$. $\phi$ is a bijection because of closedness of $G_{L}$. Let $U_{L}$ be the unit ball of $G_{L}$. By Theorem A.3.4 $U_{L}$ is weakly compact. This implies $\phi\left(U_{L}\right)$ is weakly compact and so is $\bigcup_{n=1}^{\infty} n \phi\left(U_{L}\right)=\mathcal{H}$. Now by Baire theorem, $\phi\left(U_{L}\right)$ has nonempty interior. By the proof of Theorem A.3.6, there exists $0 \leq \gamma<\infty$ such that

$$
\|f \oplus L f\| \leq \gamma\|f\| \text { for } f \in \mathcal{H} .
$$

This implies

$$
\|f \oplus L f\|^{2}=\langle\|f \oplus L f\|,\|f \oplus L f\|\rangle=\langle f, f\rangle+\langle L f, L f\rangle=\|f\|^{2}+\|L f\|^{2} \leq \gamma^{2}\|f\|^{2} .
$$

Then we obtain

$$
\|L f\| \leq\left(\gamma^{2}-1\right)^{1 / 2}\|f\|
$$

Hence $L$ is continuous.

Corollary A.3.11 ([1]). Every continuous linear bijection between Hilbert spaces has continuous inverse.

\section{A.4 Self-adjoint Operators}

Definition A.4.1. Let $\mathcal{H}$ and $\mathcal{G}$ are Hilbert spaces. A function $\Phi: \mathcal{H} \times \mathcal{G} \longrightarrow \mathbb{C}$ is a sesquilinear form (or function) if for $f, h \in \mathcal{H}, g, k \in \mathcal{G}$ and $\alpha, \beta \in \mathbb{C}$,

(i) $\Phi(\alpha f+\beta h, g)=\alpha \Phi(f, g)+\beta \Phi(h, g)$ 


$$
\text { (ii) } \Phi(f, \alpha g+\beta k)=\bar{\alpha} \Phi(f, g)+\bar{\beta} \Phi(f, k)
$$

are satisfied.

If $L \in B(\mathcal{H}, \mathcal{G})$, then the sesquilinear form $\Phi$ is defined as

$$
\Phi(f, g)=\langle L f, g\rangle_{G}
$$

and it is bounded,

$$
|\Phi(f, g)| \leq \gamma\|f\|_{\mathcal{H}}\|g\|_{\mathcal{G}} \quad \text { for } \quad f \in \mathcal{H}, g \in \mathcal{G},
$$

where $\gamma$ can take any value not less than $\|L\|$.

If we are given a sesqui-linear form $\Phi$ satisfies boundedness condition (A.22), then for fixed $f \in \mathcal{H}$, the linear functional $g \longmapsto \overline{\Phi(f, g)}$ is continuous on $\mathcal{G}$. Applying Riesz theorem, there exists uniquely $f^{\prime} \in \mathcal{G}$ such that

$$
\left\|f^{\prime}\right\|_{\mathcal{G}} \leq \gamma\|f\|_{\mathcal{H}} \text { and } \Phi(f, g)=\left\langle f^{\prime}, g\right\rangle_{\mathcal{G}} \text { for } g \in \mathcal{G} \text {. }
$$

Then $f \longmapsto f^{\prime}$ becomes linear, so we obtain $\Phi(f, g)=\left\langle f^{\prime}, g\right\rangle_{\mathcal{G}}=\langle L f, g\rangle_{\mathcal{G}}$.

Definition A.4.2. If $L \in B(\mathcal{H}, \mathcal{G})$, then there exists a unique operator in $B(\mathcal{G}, \mathcal{H})$, called adjoint of $L$, denoted by $L^{*}$ satisfying

$$
\Phi(f, g)=\left\langle f, L^{*} g\right\rangle_{\mathcal{H}} \text { for } f \in \mathcal{H}, g \in \mathcal{G} .
$$

By the definition of $L$ and $L^{*}$, we have the following relation,

$$
\langle L f, g\rangle_{\mathcal{G}}=\left\langle f, L^{*} g\right\rangle_{\mathcal{H}} \quad \text { for } f \in \mathcal{H}, g \in \mathcal{G} .
$$

Some other properties of adjoint operator:

$$
\|L\|=\left\|L^{*}\right\|
$$

Given $\mathcal{H}, \mathcal{G}$ and $\mathcal{K}$ are Hilbert spaces, if $K \in B(\mathcal{G}, \mathcal{K})$ and $L \in B(\mathcal{H}, \mathcal{G})$, then

$$
K L \in B(\mathcal{H}, \mathcal{K}) \text { and }(K L)^{*}=L^{*} K^{*} .
$$




$$
\operatorname{ker} L=\left(\operatorname{Ran} L^{*}\right)^{\perp} \text { and }(\operatorname{ker} L)^{\perp}=\operatorname{closure}\left\{\operatorname{Ran} L^{*}\right\}
$$

where $\operatorname{ker} L$ is the kernel of $L$ and $\operatorname{Ran} L$ is the range of $L$.

Theorem A.4.3 ([1]). For $L, M \in B(\mathcal{H}, \mathcal{G})$, the following assertions are mutually equivalent.

(i) $\operatorname{Ran}(M) \subseteq \operatorname{Ran}(L)$

(ii) There exists $K \in B(\mathcal{H})$ such that $M=L K$.

(iii) There exists $0 \leq \gamma<\infty$ such that

$$
\left\|M^{*} g\right\| \leq \gamma\left\|L^{*} g\right\| \quad \text { for } g \in \mathcal{G} .
$$

Definition A.4.4. Let $\mathcal{H}$ be a Hilbert space. A function $\phi: \mathcal{H} \longrightarrow \mathbb{C}$ is a quadratic form if for all $f \in \mathcal{H}$ and $\zeta \in \mathbb{C}$,

$$
\phi(\zeta f)=|\zeta|^{2} \phi(f)
$$

and

$$
\phi(f+g)+\phi(f-g)=2\{\phi(f)+\phi(g)\}
$$

are satisfied.

For $L \in B(\mathcal{H})$, the quadratic form $\phi$ on $\mathcal{H}$ is defined as

$$
\phi(f)=\langle L f, f\rangle \text { for } f \in \mathcal{H},
$$

and it is bounded

$$
|\phi(f)| \leq \gamma\|f\|^{2} \text { for } f \in \mathcal{H},
$$

where $\gamma$ can take any value not less than $\|L\|$.

The sesqui-linear form $\Phi$ associated with $L$ can be recaptured from quadratic form $\phi$ by the following equation:

$$
\Phi(f, g)=\frac{1}{4}\{\phi(f+g)-\phi(f-g)\}+\{\phi(f+i g)-\phi(f-i g)\}
$$

for all $f, g \in \mathcal{H}$. 
Definition A.4.5. A continuous linear operator $L$ on a Hilbert space $\mathcal{H}$ is said to be self-adjoint if $L=L^{*}$.

Remark A.4.6. $L$ is self adjoint if and only if the associated sesqui-linear form $\Phi$ is Hermitian.

Remark A.4.7. If $L$ is self-adjoint, norm of $L$ coincides with the minimum of $\gamma$ in (A.31) for the associated quadratic form.

Theorem A.4.8. If $L$ is a continuous self-adjoint operator, then

$$
\|L\|=\sup \{|\langle L f, f\rangle|:\|f\| \leq 1\} .
$$

Proof. Let $\gamma=\sup \{|\langle L f, f\rangle|:\|f\| \leq 1\}$.

It is obvious that $\gamma \leq\|L\|$. Consider the reverse direction. By the Remark A.4.6, since $L$ is self-adjoint, then associated quadratic form is Hermitian, so real-valued. Let fix $f, g \in \mathcal{H}$ and choose $e^{i \theta}$ such that

$$
\left\langle L\left(e^{i \theta} f\right), g\right\rangle=|\langle L f, g\rangle| .
$$

Then using the equality (A.32), it follows that

$$
\begin{aligned}
|\langle L f, g\rangle| & =\operatorname{Re}\left\langle L\left(e^{i \theta} f\right), g\right\rangle \\
& =\frac{1}{4}\left\{\left\langle L\left(e^{i \theta} f+g\right), e^{i \theta} f+g\right\rangle-\left\langle L\left(e^{i \theta} f-g\right), e^{i \theta} f-g\right\rangle\right\} \\
& \leq \frac{\gamma}{4}\left\{\left\|e^{i \theta} f+g\right\|^{2}+\left\|e^{i \theta} f-g\right\|^{2}\right\}=\frac{\gamma}{2}\left\{\|f\|^{2}+\|g\|^{2}\right\} .
\end{aligned}
$$

Now replace $f$ and $g$ by $\lambda f$ and $\frac{g}{\lambda}$ respectively, and take the minimum in $\lambda$, we obtain

$$
|\langle L f, g\rangle| \leq \gamma\|f\|\|g\| \text { for } f, g \in \mathcal{H} .
$$

Hence we have

$$
\|L\| \leq \gamma
$$

Definition A.4.9. A self-adjoint operator $L \in B(\mathcal{H})$ is said to be positive definite if $\langle L f, f\rangle \geq 0$ for all $f \in \mathcal{H}$. If $\langle L f, f\rangle=0$ only when $f=0$, then $L$ is said to be strictly positive definite. 
Remark A.4.10. For any positive definite operator $L \in B(\mathcal{H})$, a generalized Schwarz inequality holds:

$$
|\langle L f, g\rangle|^{2} \leq\langle L f, f\rangle \cdot\langle L g, g\rangle .
$$

Theorem A.4.11. Let $L$ and $M$ be continuous positive definite operators on $\mathcal{H}$ and $\mathcal{G}$ respectively. A continuous linear operator $K$ from $\mathcal{H}$ to $\mathcal{G}$ satisfies the inequality

$$
\left|\langle K f, g\rangle_{\mathcal{G}}\right|^{2} \leq\langle L f, f\rangle_{\mathcal{H}}\langle M g, g\rangle_{\mathcal{G}} \quad \text { for } \quad f \in \mathcal{H}, g \in \mathcal{G}
$$

if and only if the continuous linear operator

$$
\left[\begin{array}{ll}
L & K^{*} \\
K & M
\end{array}\right]
$$

on the direct sum Hilbert space $\mathcal{H} \oplus \mathcal{G}$ :

$$
f \oplus g \longmapsto\left(L f+K^{*} g\right) \oplus(K f+M g)
$$

is positive definite.

Proof. Suppose that (A.35) is satisfied. Let $S$ stands for the matrix

$$
\left[\begin{array}{ll}
L & K^{*} \\
K & M
\end{array}\right]
$$

such that

$$
\langle S(f \oplus g), f \oplus g\rangle=\left\langle L f+K^{*} g, f\right\rangle_{\mathcal{H}}+\langle K f+M g, g\rangle_{\mathcal{G}},
$$

then by using the property of adjoint operator and Schwarz inequality, the above equation follows as

$$
\begin{aligned}
\langle S(f \oplus g), f \oplus g\rangle & =\langle L f, f\rangle_{\mathcal{H}}+\left\langle K^{*} g, f\right\rangle_{\mathcal{H}}+\langle K f, g\rangle_{\mathcal{G}}+\langle M g, g\rangle_{\mathcal{G}} \\
& \left.=\langle L f, f\rangle_{\mathcal{H}}+\langle g, K f\rangle_{\mathcal{G}}+\langle K f, g\rangle_{\mathcal{G}}+M g, g\right\rangle_{\mathcal{G}} \\
& =\langle L f, f\rangle_{\mathcal{H}}+2 \operatorname{Re}\langle K f, g\rangle_{\mathcal{G}}+\langle M g, g\rangle_{\mathcal{G}} \\
& \geq\langle L f, f\rangle_{\mathcal{H}}+\langle M g, g\rangle_{\mathcal{G}}-2 \sqrt{\langle L f, f\rangle_{\mathcal{H}}\langle M g, g\rangle_{\mathcal{G}}} \\
& =\left\{\sqrt{\langle L f, f\rangle_{\mathcal{H}}}-\sqrt{\langle M g, g\rangle_{\mathcal{G}}}\right\}^{2} \geq 0 .
\end{aligned}
$$


Thus $\mathrm{S}$ is positive definite.

Conversely, let $\mathrm{S}$ be positive definite. By the above relation we have

$$
\begin{aligned}
0 \leq\langle S(f \oplus g), f \oplus g\rangle & =\left\langle L f+K^{*} g, f\right\rangle_{\mathcal{H}}+\langle K f+M g, g\rangle_{\mathcal{G}} \\
& =\langle L f, f\rangle_{\mathcal{H}}+\left\langle K^{*} g, f\right\rangle_{\mathcal{H}}+\langle K f, g\rangle_{\mathcal{G}}+\langle M g, g\rangle_{\mathcal{G}}
\end{aligned}
$$

then replacing $g$ with a suitable $e^{i \theta} g$, we get

$$
\langle L f, f\rangle_{\mathcal{H}}-2\left|\langle K f, g\rangle_{\mathcal{G}}\right|+\langle M g, g\rangle_{\mathcal{G}} \geq 0
$$

or

$$
\left|\langle K f, g\rangle_{\mathcal{G}}\right| \leq \frac{1}{2}\left\{\langle L f, f\rangle_{\mathcal{H}}+\langle M g, g\rangle_{\mathcal{G}}\right\} .
$$

Then replacing $f$ and $g$ by $\lambda f$ and $\frac{g}{\lambda}$ respectively, we obtain

$$
\left|\langle K f, g\rangle_{\mathcal{G}}\right|^{2} \leq\langle L f, f\rangle_{\mathcal{H}}\langle M g, g\rangle_{\mathcal{G}} .
$$

Theorem A.4.12 ([1]). Let $L$ be a continuous positive definite operator. Then there exists uniquely a positive definite operator called the square root of $L$, denoted by $L^{1 / 2}$, such that $\left(L^{1 / 2}\right)^{2}=L$.

Definition A.4.13. For a continuous linear operator $L$, the square root of the positive definite operator $L^{*} L$ is called the modulus (operator) of $L$.

Definition A.4.14. A linear operator $V$ between Hilbert spaces $\mathcal{H}$ and $\mathcal{G}$ is called isometric or an isometry if it preserves the norm, that is,

$$
\|V f\|_{\mathcal{G}}=\|f\|_{\mathcal{H}} \quad \text { for } \quad f \in \mathcal{H} .
$$

By (A.36), a continuous linear operator $V$ is isometric if and only if $V^{*} V=I_{\mathcal{H}}$, in other words, $V$ preserves inner product :

$$
\langle V f, V g\rangle_{\mathcal{G}}=\langle f, g\rangle_{\mathcal{H}} \text { for } f, g \in \mathcal{H}
$$

Definition A.4.15. A linear operator $U: \mathcal{H} \longrightarrow \mathcal{H}$ that is a surjective isometry is called a unitary (operator). 
If $U \in B(H)$ is a unitary operator, then $U^{*}=U^{-1}$.

Definition A.4.16. A continuous linear operator $U$ between Hilbert spaces $\mathcal{H}$ and $\mathcal{G}$ is called a partial isometry if for $f \in(\operatorname{ker} U)^{\perp}=\operatorname{Ran}\left(U^{*}\right),\|U f\|=\|f\|$. The space $(\operatorname{ker} U)^{\perp}$ is called the initial space of $U$ and the space $\operatorname{Ran}(\mathrm{U})$ is called the final space of $U$.

For a partial isometry $U$, its adjoint $U^{*}$ is again a partial isometry.

Corollary A.4.17 ([1]). Each continuous linear operator $L$ on $\mathcal{H}$ admits a unique decomposition

$$
L=U \tilde{L},
$$

where $\tilde{L}$ is positive definite and $U$ is a partial isometry with initial space the closure of $\operatorname{Ran}(\tilde{L})$. Indeed, $\tilde{L}$ must be the modulus $|L|$. 


\section{Bibliography}

[1] T. Ando, Reproducing Kernel Spaces and Quadratic Inequalities, Lecture Notes, Hokkaido University, Research Institute of Applied Electricity, Division of Applied Mathematics, Sapporo, Japan, 1987.

[2] N. Aronszajn, Theory of reproducing kernels, Trans. Amer. Math. Soc. 68(1950), 337-404.

[3] J.B. Conway, A Course in Functional Analysis, Springer Verlag, Berlin Heidelberg - New York, 1989.

[4] P.L. Duren, Theory of $H_{p}$ Spaces, Academic Press, INC., New York, 1970.

[5] P.L. Duren, A. Schuster, Bergman Spaces, Amer. Math. Soc., Providence R.I. 2004.

[6] H. Dym, J-Contractive Matrix Functions, Reproducing Kernel Hilbert Spaces and Interpolation, CBMS Regional Conference Series in Mathematics, Amer. Math. Soc., Providence R.I. 1989.

[7] P. Koosis, Introduction to $H_{p}$ Spaces, Cambridge Mathematical Press, Cambridge, 1970.

[8] S. Saiton, Theory of Reproducing Kernels and its Applications, Pitman Research Notes in Mathematics Series, vol. 189, Longman Scientific and Technical, Harlow, 1988.

[9] J. Weidman, Linear Operators in Hilbert Spaces, Springer Verlag, Berlin Heidelberg - New York, 1980. 